\title{
OSCILLATIONS AND CONCENTRATIONS IN SEQUENCES OF GRADIENTS*
}

\author{
Agnieszka KaŁAmajskA ${ }^{1}$ And Martin KruŽÍK ${ }^{2,3}$
}

\begin{abstract}
We use DiPerna's and Majda's generalization of Young measures to describe oscillations and concentrations in sequences of gradients, $\left\{\nabla u_{k}\right\}$, bounded in $L^{p}\left(\Omega ; \mathbb{R}^{m \times n}\right)$ if $p>1$ and $\Omega \subset \mathbb{R}^{n}$ is a bounded domain with the extension property in $W^{1, p}$. Our main result is a characterization of those DiPerna-Majda measures which are generated by gradients of Sobolev maps satisfying the same fixed Dirichlet boundary condition. Cases where no boundary conditions nor regularity of $\Omega$ are required and links with lower semicontinuity results by Meyers and by Acerbi and Fusco are also discussed.
\end{abstract}

Mathematics Subject Classification. 49J45, 35B05.

Received July 18, 2006.

Published online September 21, 2007.

\section{INTRODUCTION}

Oscillations and/or concentrations appear in many problems in the calculus of variations, partial differential equations, or optimal control theory, which admit only $L^{p}$ but not $L^{\infty}$ apriori estimates; $c f$. [17,27]. While Young measures [43] successfully capture oscillatory behavior of sequences they completely miss concentrations. There are several tools how to deal with concentrations. They can be considered as generalization of Young measures, see for example DiPerna's and Majda's treatment of concentrations [9], Alibert's and Bouchitté's approach [2] or Fonseca's method described in [13]. An overview can be found in [36,40]. In many cases we are interested in oscillation/concentration effects generated by sequences of gradients. A characterization of Young measures generated by gradients was completely given by Kinderlehrer and Pedregal [21,22], cf. also [32,33]. To our knowledge, the first attempt to characterize both oscillations and concentrations in sequences of gradients is due to Fonseca, Müller, and Pedregal [14]. They describe concentrations by means of a varifold while oscillations by gradient Young measures, following the works $[3,4,13,35]$. The authors give necessary and sufficient conditions on the varifold, so that they can fully describe effects of concentrations and oscillations on

Keywords and phrases. Sequences of gradients, concentrations, oscillations, quasiconvexity.

* The work of A.K. was supported by the KBN grant No. 1-PO3A-008-29 and partially supported by EC FP6 M. Curie ToK program SPADEZ, MTKD-CT-2004-01458 and by MNiSW SPB, while M.K. was supported by the grants IAA 1075402 (GA $A V \check{C} R$ ) and $V Z 6840770021$ (M $\breve{S} M T \check{C} R$ ).

1 Institute of Mathematics, Warsaw University, ul. Banacha 2, 02-097 Warsaw, Poland; Agnieszka.Kalamajska@mimuw.edu.pl This research was done while A.K. was visiting Institute of Mathematics of the Polish Academy of Sciences at Warsaw in the academic year $2004 / 2005$.

2 Institute of Information Theory and Automation, Academy of Sciences of the Czech Republic.

Corresponding address Pod vodárenskou věží 4, 18208 Praha 8, Czech Republic.

${ }^{3}$ Faculty of Civil Engineering, Czech Technical University, Thákurova 7, 16629 Praha 6, Czech Republic; kruzik@utia.cas.cz (corresponding author).

(c) EDP Sciences, SMAI 2007 
sequences of integrands $\left\{g(x) v\left(\nabla u_{k}(x)\right)\right\}_{k \in \mathbb{N}}$ where $1<p<\infty,\left\{u_{k}\right\}_{k \in \mathbb{N}} \subset W^{1, p}\left(\Omega ; \mathbb{R}^{m}\right)$ (where $W^{1, p}\left(\Omega ; \mathbb{R}^{m}\right)$ is the classical Sobolev space of $\mathbb{R}^{m}$-valued functions), $v /\left(1+|\cdot|^{p}\right)$ is a real-valued function and has a continuous extension on the compactification of $\mathbb{R}^{m \times n}$ by the sphere, and $g: \Omega \rightarrow \mathbb{R}$ is continuous and vanishes on the boundary of a bounded domain $\Omega \subset \mathbb{R}^{n}$.

In this paper we deal with general DiPerna-Majda measures generated by gradients of functions commonly bounded in $W^{1, p}\left(\Omega ; \mathbb{R}^{m}\right)$. They encode oscillation and concentration effects in sequences of compositions like $\left\{g(x) v\left(\nabla u_{k}(x)\right)\right\}$ in a general case when the function $v(s) /\left(1+|s|^{p}\right)$ has a continuous extension on an arbitrary metrizable compactification of $\mathbb{R}^{m \times n}$ and $g: \bar{\Omega} \rightarrow \mathbb{R}$ is continuous and does not necessarily vanish on the boundary of $\Omega$. This allows to study concentrations and oscillation effects for the more general class of functions $v$ admitted to the compositions with sequences $\left\{\nabla u_{k}\right\}_{k \in \mathbb{N}}$. For example the function $v_{0}(\lambda)=\sin (|\lambda|)$ is continuous on $\mathbb{R}^{n}$ but it cannot be continuously extended to the compactification of $\mathbb{R}^{m \times n}$ by the sphere (considered in [14]) as the limits $\lim _{t \rightarrow \infty} v_{0}(t \theta)$ where $\theta$ belongs to the unit sphere in $\mathbb{R}^{m \times n}$ do not exist. Here we study the oscillations and concentrations of sequences like $\left\{g(x) v_{0}\left(\nabla u_{k}(x)\right)\left(1+\left|\nabla u_{k}\right|^{p}\right)\right\}_{k \in \mathbb{N}}$ as well. Also, the assumption that $g$ does not need to vanish on the boundary of $\Omega$ allows us to study concentrations of sequences on the boundary of $\Omega$.

Our main result is the characterization of those DiPerna-Majda measures which are generated by gradients of Sobolev functions (bounded in $W^{1, p}\left(\Omega ; \mathbb{R}^{m}\right)$ ), where $1<p<\infty$, with the same Dirichlet boundary data on the boundary of $\Omega$, provided that $\Omega$ is a bounded domain with an extension property in $W^{1, p}$. Here we solve the case $1<p<+\infty$. Meanwhile $p=+\infty$ excludes concentrations and is completely described by gradient Young measures [21]. The case $p=1$ seems to be much more involved because of the loss of reflexivity. We also derive the necessary conditions (for $1<p<\infty$ ) for those DiPerna-Majda measures which are generated by gradients of Sobolev mappings with no prescribed boundary conditions for an arbitrary bounded domain $\Omega$. As an application of our techniques we derive new lower semicontinuity results (Th. 2.9) for variational functionals, generalizing some variants of Acerbi and Fusco theorem (see e.g. $[1,18,28]$ and references therein). We also obtain some variants of the lower semicontinuity results obtained previously by Meyers, [30]; $c f$. Theorem 2.10 .

Let us mention that a few of our results seem to be of an independent interest. Particularly, it is Lemma 3.5 and Lemma 4.1 showing local and averaging properties of DiPerna-Majda measures, respectively.

Our methods are based on powerful techniques introduced in [21] and [22] to obtain the explicit characterization of Young measures generated by gradients. We also benefit from the characterization of DiPerna-Majda measures generated by unconstrained sequences given in [25], see also [26] where numerical issues are discussed in detail.

\section{Preliminaries And Result statements}

\subsection{Basic notation}

Let us start with a few definitions and with the explanation of our notation. Having a bounded domain $\Omega \subset \mathbb{R}^{n}$ we denote by $C(\Omega)$ the space of continuous functions defined on $\Omega$. In the sequel, $M_{g}$ means the continuity modulus of $g \in C(\bar{\Omega})$. In what follows $\operatorname{rca}(S)$ denotes the set of regular countably additive set functions on the Borel $\sigma$-algebra on a metrizable set $S$ (cf. [10]), its subset, $\operatorname{rca}_{1}^{+}(S)$, denotes regular probability measures on a set $S$. We write " $\gamma$-almost all" or " $\gamma$-a.e." if we mean "up to a set with the $\gamma$-measure zero". If $\gamma$ is the $n$-dimensional Lebesgue measure we omit writing $\gamma$ in the notation. The support of a measure $\sigma \in \operatorname{rca}(\Omega)$ is the smallest closed set $S$ such that $\sigma(A)=0$ if $S \cap A=\emptyset$. If $\sigma \in \operatorname{rca}(\bar{\Omega})$ we write $\sigma_{s}$ and $d_{\sigma}$ for the singular part and density of $\sigma$ defined by the Lebesgue decomposition (with respect to the Lebesgue measure), respectively. We denote by 'w-lim' or by $\rightarrow$ the weak limit. Analogously we indicate weak* limits.

If $\Omega$ is a Borel subset of $\mathbb{R}^{n}, \mu \in \operatorname{rca}^{+}(\Omega)$ and $u \in L^{1}(\Omega, \mu)$ by $\mathcal{L}_{u}^{\mu}$ we denote the set of all Lebesgue points of $u$ with respect to $\mu$. If $\mu$ is the Lebesgue measure we simply write $\mathcal{L}_{u}$.

If not said otherwise, we will suppose in the sequel that $\Omega \subset \mathbb{R}^{n}$ is a bounded domain with a Lipschitz boundary (however, generalizations to less regular domains are possible). 
By $L^{p}(\Omega, \mu)$ we denote the usual Lebesgue space equipped with the measure $\mu$. We omit $\mu$ if it is the Lebesgue measure. Further, $W^{1, p}\left(\Omega ; \mathbb{R}^{m}\right)$ where $1 \leq p<+\infty$ denotes the usual Sobolev space (of $\mathbb{R}^{m}$-valued functions) and $W_{0}^{1, p}\left(\Omega ; \mathbb{R}^{m}\right)$ denotes the completion of $C_{0}^{\infty}\left(\Omega, \mathbb{R}^{m}\right)$ (smooth functions with bounded support) in $W^{1, p}\left(\Omega ; \mathbb{R}^{m}\right.$ ) (see e.g. [29]). If $m=1$ then $\mathbb{R}^{m}$ is omitted from the notation. We say that $\Omega$ has the extension property in $W^{1, p}$ if every function $u \in W^{1, p}(\Omega)$ can be extended outside $\Omega$ to $\tilde{u} \in W^{1, p}\left(\mathbb{R}^{n}\right)$ and the extension operator is linear and bounded. If $\Omega$ is an arbitrary domain and $u, w \in W^{1, p}\left(\Omega, \mathbb{R}^{m}\right)$ we say that $u=w$ on $\partial \Omega$ if $u-w \in W_{0}^{1, p}\left(\Omega ; \mathbb{R}^{m}\right)$.

\subsection{Quasiconvex functions}

Let $\Omega \subset \mathbb{R}^{n}$ be a bounded regular domain. We say that a function $v: \mathbb{R}^{m \times n} \rightarrow \mathbb{R}$ is quasiconvex if for any $s_{0} \in \mathbb{R}^{m \times n}$ and any $\varphi \in W_{0}^{1, \infty}\left(\Omega ; \mathbb{R}^{m}\right)$

$$
v\left(s_{0}\right)|\Omega| \leq \int_{\Omega} v\left(s_{0}+\nabla \varphi(x)\right) \mathrm{d} x .
$$

If $v: \mathbb{R}^{m \times n} \rightarrow \mathbb{R}$ is not quasiconvex we define its quasiconvex envelope $Q v: \mathbb{R}^{m \times n} \rightarrow \mathbb{R}$ as

$$
Q v(s)=\sup \left\{h(s) ; h \leq v ; h: \mathbb{R}^{m \times n} \rightarrow \mathbb{R} \text { quasiconvex }\right\}
$$

and we put $Q v=-\infty$ if the set on the right-hand side of (2.1) is empty. If $v$ is locally bounded and Borel measurable then for any $s_{0} \in \mathbb{R}^{m \times n}$ (see [8])

$$
Q v\left(s_{0}\right)=\inf _{\varphi \in W_{0}^{1, \infty}\left(\Omega ; \mathbb{R}^{m}\right)} \frac{1}{|\Omega|} \int_{\Omega} v\left(s_{0}+\nabla \varphi(x)\right) \mathrm{d} x .
$$

If $|v(s)| \leq C\left(1+|s|^{p}\right)$ for some $C>0$ and all $s \in \mathbb{R}^{m \times n}$ then equivalently

$$
Q v\left(s_{0}\right)=\inf _{\varphi \in W_{0}^{1, p}\left(\Omega ; \mathbb{R}^{m}\right)} \frac{1}{|\Omega|} \int_{\Omega} v\left(s_{0}+\nabla \varphi(x)\right) \mathrm{d} x,
$$

as pointed out in [14]. We refer to [6] for the notion of $W^{1, p}$-quasiconvexity.

Let us point out that

$$
Q v\left(s_{0}\right)=\inf _{\varphi \in W_{s_{0}}^{1, p}\left(\Omega ; \mathbb{R}^{m}\right)} \frac{1}{|\Omega|} \int_{\Omega} v(\nabla \varphi(x)) \mathrm{d} x,
$$

where $W_{s_{0}}^{1, p}\left(\Omega ; \mathbb{R}^{m}\right)=\left\{\varphi \in W^{1, p}\left(\Omega ; \mathbb{R}^{m}\right) ; \varphi(x)=s_{0} x\right.$ on $\left.\partial \Omega\right\}$.

We will also need the following elementary result. It can be found in a more general form e.g. in [8], Chapter 4, Lemma 2.2 , or in [31].

Lemma 2.1. Let $v: \mathbb{R}^{m \times n} \rightarrow \mathbb{R}$ be quasiconvex with $|v(s)| \leq C\left(1+|s|^{p}\right), 1 \leq p<+\infty, C>0$, for all $s \in \mathbb{R}^{m \times n}$. Then there is a constant $\alpha \geq 0$ such that for every $s_{1}, s_{2} \in \mathbb{R}^{m \times n}$ it holds

$$
\left|v\left(s_{1}\right)-v\left(s_{2}\right)\right| \leq \alpha\left(1+\left|s_{1}\right|^{p-1}+\left|s_{2}\right|^{p-1}\right)\left|s_{1}-s_{2}\right| .
$$




\subsection{Young measures}

For $p \geq 0$ we define the following subspace of the space $C\left(\mathbb{R}^{m \times n}\right)$ of all continuous functions on $\mathbb{R}^{m \times n}$ :

$$
C_{p}\left(\mathbb{R}^{m \times n}\right)=\left\{v \in C\left(\mathbb{R}^{m \times n}\right) ; v(s)=o\left(|s|^{p}\right) \text { for }|s| \rightarrow \infty\right\} .
$$

The Young measures on a bounded domain $\Omega \subset \mathbb{R}^{n}$ are weakly* measurable mappings $x \mapsto \nu_{x}: \Omega \rightarrow$ $\operatorname{rca}\left(\mathbb{R}^{m \times n}\right)$ with values in probability measures; and the adjective "weakly* measurable" means that, for any $v \in C_{0}\left(\mathbb{R}^{m \times n}\right)$, the mapping $\Omega \rightarrow \mathbb{R}: x \mapsto\left\langle\nu_{x}, v\right\rangle=\int_{\mathbb{R}^{m \times n}} v(\lambda) \nu_{x}(\mathrm{~d} \lambda)$ is measurable in the usual sense. Let us remind that, by the Riesz theorem the space $\operatorname{rca}\left(\mathbb{R}^{m \times n}\right)$, normed by the total variation, is a Banach space which is isometrically isomorphic with $C_{0}\left(\mathbb{R}^{m \times n}\right)^{*}$, where $C_{0}\left(\mathbb{R}^{m \times n}\right)$ stands for the space of all continuous functions $\mathbb{R}^{m \times n} \rightarrow \mathbb{R}$ vanishing at infinity. Let us denote the set of all Young measures by $\mathcal{Y}\left(\Omega ; \mathbb{R}^{m \times n}\right)$. It is known that $\mathcal{Y}\left(\Omega ; \mathbb{R}^{m \times n}\right)$ is a convex subset of $L_{\mathrm{w}}^{\infty}\left(\Omega ; \mathrm{rca}\left(\mathbb{R}^{m \times n}\right)\right) \cong L^{1}\left(\Omega ; C_{0}\left(\mathbb{R}^{m \times n}\right)\right)^{*}$, where the subscript "w" indicates the property "weakly* measurable". A classical result $[39,42]$ is that, for every sequence $\left\{y_{k}\right\}_{k \in \mathbb{N}}$ bounded in $L^{\infty}\left(\Omega ; \mathbb{R}^{m \times n}\right)$, there exists its subsequence (denoted by the same indices for notational simplicity) and a Young measure $\nu=\left\{\nu_{x}\right\}_{x \in \Omega} \in \mathcal{Y}\left(\Omega ; \mathbb{R}^{m \times n}\right)$ such that

$$
\forall v \in C_{0}\left(\mathbb{R}^{m \times n}\right): \quad \lim _{k \rightarrow \infty} v \circ y_{k}=v_{\nu} \quad \text { weakly* in } L^{\infty}(\Omega),
$$

where $\left[v \circ y_{k}\right](x)=v\left(y_{k}(x)\right)$ and

$$
v_{\nu}(x)=\int_{\mathbb{R}^{m \times n}} v(\lambda) \nu_{x}(\mathrm{~d} \lambda) .
$$

Let us denote by $\mathcal{Y}^{\infty}\left(\Omega ; \mathbb{R}^{m \times n}\right)$ the set of all Young measures which are created by this way, i.e. by taking all bounded sequences in $L^{\infty}\left(\Omega ; \mathbb{R}^{m \times n}\right)$. Note that (2.4) actually holds for any $v: \mathbb{R}^{m \times n} \rightarrow \mathbb{R}$ continuous.

A generalization of this result was formulated by Schonbek [37] (cf. also [5]): if $1 \leq p<+\infty$ : for every sequence $\left\{y_{k}\right\}_{k \in \mathbb{N}}$ bounded in $L^{p}\left(\Omega ; \mathbb{R}^{m \times n}\right)$ there exists its subsequence (denoted by the same indices) and a Young measure $\nu=\left\{\nu_{x}\right\}_{x \in \Omega} \in \mathcal{Y}\left(\Omega ; \mathbb{R}^{m \times n}\right)$ such that

$$
\forall v \in C_{p}\left(\mathbb{R}^{m \times n}\right): \quad \lim _{k \rightarrow \infty} v \circ y_{k}=v_{\nu} \quad \text { weakly in } L^{1}(\Omega) .
$$

We say that $\left\{y_{k}\right\}$ generates $\nu$ if (2.6) holds.

Let us denote by $\mathcal{Y}^{p}\left(\Omega ; \mathbb{R}^{m \times n}\right)$ the set of all Young measures which are created by this way, i.e. by taking all bounded sequences in $L^{p}\left(\Omega ; \mathbb{R}^{m \times n}\right)$. The subset of $\mathcal{Y}^{p}\left(\Omega ; \mathbb{R}^{m \times n}\right)$ containing Young measures generated by gradients of $W^{1, p}\left(\Omega ; \mathbb{R}^{m}\right)$ maps will be denoted by $\mathcal{G} \mathcal{Y}^{p}\left(\Omega ; \mathbb{R}^{m \times n}\right)$.

We will use the following lemma from [14] concerning Young measures from $\mathcal{Y}^{p}\left(\Omega ; \mathbb{R}^{m \times n}\right)$ which are generated by sequences of gradients. A similar result was also proved by Kristensen [23].

Lemma 2.2. Let $1<p<+\infty$ and $\Omega \subset \mathbb{R}^{n}$ be an open bounded set and let $\left\{u_{k}\right\}_{k \in \mathbb{N}} \subset W^{1, p}\left(\Omega ; \mathbb{R}^{m}\right)$ be bounded. Then there is a subsequence $\left\{u_{j}\right\}_{j \in \mathbb{N}}$ and a sequence $\left\{z_{j}\right\}_{j \in \mathbb{N}} \subset W^{1, p}\left(\Omega ; \mathbb{R}^{m}\right)$ such that

$$
\lim _{j \rightarrow \infty} \mid\left\{x \in \Omega ; z_{j}(x) \neq u_{j}(x) \text { or } \nabla z_{j}(x) \neq \nabla u_{j}(x)\right\} \mid=0
$$

and $\left\{\left|\nabla z_{j}\right|^{p}\right\}_{j \in \mathbb{N}}$ is relatively weakly compact in $L^{1}(\Omega)$. In particular, $\left\{\nabla u_{j}\right\}$ and $\left\{\nabla z_{j}\right\}$ generate the same Young measure. 


\subsection{DiPerna-Majda measures}

\subsubsection{Definition and basic properties}

Let $\mathcal{R}$ be a complete (i.e. containing constants, separating points from closed subsets and closed with respect to the Chebyshev norm) separable ring of continuous bounded functions $\mathbb{R}^{m \times n} \rightarrow \mathbb{R}$. It is known [11] Section 3.12.21, that there is a one-to-one correspondence $\mathcal{R} \leftrightarrow \beta_{\mathcal{R}} \mathbb{R}^{m \times n}$ between such rings and metrizable compactifications of $\mathbb{R}^{m \times n}$; by a compactification we mean here a compact set, denoted by $\beta_{\mathcal{R}} \mathbb{R}^{m \times n}$, into which $\mathbb{R}^{m \times n}$ is embedded homeomorphically and densely. For simplicity, we will not distinguish between $\mathbb{R}^{m \times n}$ and its image in $\beta_{\mathcal{R}} \mathbb{R}^{m \times n}$. Similarly, we will not distinguish between elements of $\mathcal{R}$ and their unique continuous extensions defined on $\beta_{\mathcal{R}} \mathbb{R}^{m \times n}$. This means that if $i: \mathbb{R}^{m \times n} \rightarrow \beta_{\mathcal{R}} \mathbb{R}^{m \times n}$ is the homeomorphic embedding and $v_{0} \in \mathcal{R}$ then the same notation is used also for $v_{0} \circ i^{-1}: i\left(\mathbb{R}^{m \times n}\right) \rightarrow \mathbb{R}$ and for its unique continuous extension to $\beta_{\mathcal{R}} \mathbb{R}^{m \times n}$.

Let $\sigma \in \operatorname{rca}(\bar{\Omega})$ be a positive Radon measure on a bounded domain $\Omega \subset \mathbb{R}^{n}$. A mapping $\hat{\nu}: x \mapsto \hat{\nu}_{x}$ belongs to the space $L_{\mathrm{w}}^{\infty}\left(\bar{\Omega}, \sigma ; \operatorname{rca}\left(\beta_{\mathcal{R}} \mathbb{R}^{m \times n}\right)\right)$ if it is weakly* $\sigma$-measurable $\left(i . e\right.$., for any $v_{0} \in C_{0}\left(\mathbb{R}^{m \times n}\right)$, the mapping $\bar{\Omega} \rightarrow \mathbb{R}: x \mapsto \int_{\beta_{\mathcal{R}} \mathbb{R}^{m \times n}} v_{0}(s) \hat{\nu}_{x}(\mathrm{~d} s)$ is $\sigma$-measurable in the usual sense). If additionally $\hat{\nu}_{x} \in \mathrm{rca}_{1}^{+}\left(\beta_{\mathcal{R}} \mathbb{R}^{m \times n}\right)$ for $\sigma$-a.a. $x \in \bar{\Omega}$ the collection $\left\{\hat{\nu}_{x}\right\}_{x \in \bar{\Omega}}$ is the so-called Young measure on $(\bar{\Omega}, \sigma)([43]$, see also $[5,36,39,41,42])$.

DiPerna and Majda [9] shown that having a bounded sequence in $L^{p}\left(\Omega ; \mathbb{R}^{m \times n}\right)$ with $1 \leq p<+\infty$ defined on an open domain $\Omega \subseteq \mathbb{R}^{n}$, there exists its subsequence (denoted by the same indices) a positive Radon measure $\sigma \in \operatorname{rca}(\bar{\Omega})$ and a Young measure $\hat{\nu}: x \mapsto \hat{\nu}_{x}$ on $(\bar{\Omega}, \sigma)$ such that $(\sigma, \hat{\nu})$ is attainable by a sequence $\left\{y_{k}\right\}_{k \in \mathbb{N}} \subset L^{p}\left(\Omega ; \mathbb{R}^{m \times n}\right)$ in the sense that $\forall g \in C(\bar{\Omega}) \forall v_{0} \in \mathcal{R}$ :

$$
\lim _{k \rightarrow \infty} \int_{\Omega} g(x) v\left(y_{k}(x)\right) \mathrm{d} x=\int_{\bar{\Omega}} g(x) \int_{\beta_{\mathcal{R}} \mathbb{R}^{m \times n}} v_{0}(s) \hat{\nu}_{x}(\mathrm{~d} s) \sigma(\mathrm{d} x),
$$

where

$$
v \in \Upsilon_{\mathcal{R}}^{p}\left(\mathbb{R}^{m \times n}\right):=\left\{v_{0}\left(1+|\cdot|^{p}\right) ; v_{0} \in \mathcal{R}\right\}
$$

In particular, putting $v_{0}=1 \in \mathcal{R}$ in $(2.8)$ we can see that

$$
\lim _{k \rightarrow \infty}\left(1+\left|y_{k}\right|^{p}\right)=\sigma \quad \text { weakly* in } \operatorname{rca}(\bar{\Omega}) .
$$

If $(2.8)$ holds, we say that $\left\{y_{k}\right\} \in \mathbb{N}$ generates $(\sigma, \hat{\nu})$. Let us denote by $\mathcal{D} \mathcal{M}_{\mathcal{R}}^{p}\left(\Omega ; \mathbb{R}^{m \times n}\right)$ the set of all pairs $(\sigma, \hat{\nu}) \in \operatorname{rca}(\bar{\Omega}) \times L_{\mathrm{w}}^{\infty}\left(\bar{\Omega}, \sigma ; \operatorname{rca}\left(\beta_{\mathcal{R}} \mathbb{R}^{m \times n}\right)\right)$ attainable by sequences from $L^{p}\left(\Omega ; \mathbb{R}^{m \times n}\right)$; note that, taking $v_{0}=1$ in (2.8), one can see that these sequences must be inevitably bounded in $L^{p}\left(\Omega ; \mathbb{R}^{m \times n}\right)$. The explicit description of the elements from $\mathcal{D} \mathcal{M}_{\mathcal{R}}^{p}\left(\Omega ; \mathbb{R}^{m \times n}\right)$, called DiPerna-Majda measures, for unconstrained sequences was done in [25], Theorem 2.

Alternatively, DiPerna and Majda [9] worked with measures from $\operatorname{rca}\left(\bar{\Omega} \times \beta_{\mathcal{R}} \mathbb{R}^{m \times n}\right)$; let us put here

$$
\begin{aligned}
& \operatorname{DM}_{\mathcal{R}}^{p}\left(\Omega ; \mathbb{R}^{m \times n}\right)=\left\{\eta \in \operatorname{rca}\left(\bar{\Omega} \times \beta_{\mathcal{R}} \mathbb{R}^{m \times n}\right) ; \exists\left\{y_{k}\right\}_{k \in \mathbb{N}} \subset L^{p}\left(\Omega ; \mathbb{R}^{m \times n}\right)\right. \\
& \left.\forall h_{0} \in C\left(\bar{\Omega} \times \beta_{\mathcal{R}} \mathbb{R}^{m \times n}\right):\left\langle\eta, h_{0}\right\rangle=\lim _{k \rightarrow \infty} \int_{\Omega} h_{0}\left(x, y_{k}(x)\right)\left(1+\left|y_{k}(x)\right|^{p}\right) \mathrm{d} x\right\} .
\end{aligned}
$$

Let $\eta \in \operatorname{DM}_{\mathcal{R}}^{p}\left(\Omega ; \mathbb{R}^{m \times n}\right)$ be generated by $\left\{y_{k}\right\}_{k \in \mathbb{N}} \subset L^{p}\left(\Omega ; \mathbb{R}^{m \times n}\right)$, i.e. $\left\langle\eta, h_{0}\right\rangle=\lim _{k \rightarrow \infty} \int_{\Omega} h_{0}\left(x, y_{k}(x)\right)\left(1+\left|y_{k}(x)\right|^{p}\right) \mathrm{d} x$ whenever $h_{0} \in C\left(\bar{\Omega} \times \beta_{\mathcal{R}} \mathbb{R}^{m \times n}\right)$. Then there is a uniquely defined $(\sigma, \hat{\nu}) \in \mathcal{D} \mathcal{M}_{\mathcal{R}}^{p}\left(\Omega ; \mathbb{R}^{m \times n}\right)$ such that

$$
\left\langle\eta, h_{0}\right\rangle=\int_{\bar{\Omega}} \int_{\beta_{\mathcal{R}} \mathbb{R}^{m \times n}} h_{0}(x, s) \hat{\nu}_{x}(\mathrm{~d} s) \sigma(\mathrm{d} x),
$$


if $h_{0} \in C\left(\bar{\Omega} \times \beta_{\mathcal{R}} \mathbb{R}^{m \times n}\right)$. Indeed, let at first $h_{0} \in V:=\left\{g(x) v_{0}(s): g \in C(\bar{\Omega}), v_{0} \in \mathcal{R}\right\}$. For each subsequence of $\left\{y_{k}\right\}_{k \in \mathbb{N}}$ which generates some DiPerna-Majda measure the limit of $\lim _{k \rightarrow \infty} \int_{\Omega} h_{0}\left(x, y_{k}(x)\right)\left(1+\left|y_{k}(x)\right|^{p}\right) \mathrm{d} x$ is the same and equal $\left\langle\eta, h_{0}\right\rangle$. Therefore the whole sequence must generate some DiPerna Majda measure which we denote by $(\sigma, \hat{\nu})$ and (2.11) is true for all $h_{0} \in V$. By the Stone-Weierstrass theorem the set $V$ is linearly dense in $C\left(\bar{\Omega} \times \beta_{\mathcal{R}} \mathbb{R}^{m \times n}\right)$ (it forms an algebra and separates points). Therefore (2.11) holds true for every $h_{0} \in C\left(\bar{\Omega} \times \beta_{\mathcal{R}} \mathbb{R}^{m \times n}\right)$. On the other hand if $\left\{y_{k}\right\}_{k \in \mathbb{N}}$ generates some $(\sigma, \hat{\nu}) \in \mathcal{D} \mathcal{M}_{\mathcal{R}}^{p}\left(\Omega ; \mathbb{R}^{m \times n}\right)$ then it also generates some $\eta \in \operatorname{DM}_{\mathcal{R}}^{p}\left(\Omega ; \mathbb{R}^{m \times n}\right)$ and moreover, $\eta$ fulfills the identity (2.11). The proof of this fact follows from (2.8) and the density of the linear hull of $V$ in $C\left(\bar{\Omega} \times \beta_{\mathcal{R}} \mathbb{R}^{m \times n}\right)$. Therefore, (2.8) can be generalized to

$$
\lim _{k \rightarrow \infty} \int_{\Omega} h_{0}\left(x, y_{k}(x)\right)\left(1+\left|y_{k}\right|^{p}\right) \mathrm{d} x=\int_{\bar{\Omega}} \int_{\beta_{\mathcal{R}} \mathbb{R}^{m \times n}} h_{0}(x, s) \hat{\nu}_{x}(\mathrm{~d} s) \sigma(\mathrm{d} x),
$$

whenever $\left\{y_{k}\right\}_{k \in \mathbb{N}}$ generates $(\sigma, \hat{\nu})$ and $h_{0} \in C\left(\bar{\Omega} \times \beta_{\mathcal{R}} \mathbb{R}^{m \times n}\right)$.

Without causing any misunderstanding, the elements of $\operatorname{DM}_{\mathcal{R}}^{p}\left(\Omega ; \mathbb{R}^{m \times n}\right)$ will be addressed as DiPerna-Majda measures too and we write $\eta \cong(\sigma, \hat{\nu})$ for $\eta \in \operatorname{DM}_{\mathcal{R}}^{p}\left(\Omega ; \mathbb{R}^{m \times n}\right)$ and $(\sigma, \hat{\nu}) \in \mathcal{D M}_{\mathcal{R}}^{p}\left(\Omega ; \mathbb{R}^{m \times n}\right)$ if $(2.11)$ holds for any $h_{0} \in C\left(\bar{\Omega} \times \beta_{\mathcal{R}} \mathbb{R}^{m \times n}\right)$. It is sufficient to verify it for $h_{0} \in V$.

It is known (see [36]) that $\operatorname{DM}_{\mathcal{R}}^{p}\left(\Omega ; \mathbb{R}^{m \times n}\right)$ is a convex, closed, non-compact but locally compact and locally sequentially compact subset of the locally convex space $\operatorname{rca}\left(\bar{\Omega} \times \beta_{\mathcal{R}} \mathbb{R}^{m \times n}\right)$ considered in its weak* topology.

Note that for $\left(\sigma^{j}, \hat{\nu}^{j}\right),(\sigma, \hat{\nu}) \in \mathcal{D M}_{\mathcal{R}}^{p}\left(\Omega ; \mathbb{R}^{m \times n}\right)$ the sequence $\left\{\left(\sigma^{j}, \hat{\nu}^{j}\right)\right\}_{j \in \mathbb{N}}$ converges weakly* to $(\sigma, \hat{\nu})$ if

$$
\int_{\bar{\Omega}} \int_{\beta_{\mathcal{R}} \mathbb{R}^{m \times n}} h_{0}(x, s) \hat{\nu}_{x}^{j}(\mathrm{~d} s) \sigma^{j}(\mathrm{~d} x) \rightarrow \int_{\bar{\Omega}} \int_{\beta_{\mathcal{R}} \mathbb{R}^{m \times n}} h_{0}(x, s) \hat{\nu}_{x}(\mathrm{~d} s) \sigma(\mathrm{d} x)
$$

for every $h_{0} \in C\left(\bar{\Omega} \times \beta_{\mathcal{R}} \mathbb{R}^{m \times n}\right)$. We denote this convergence by $\left(\sigma^{j}, \hat{\nu}^{j}\right) \rightarrow(\sigma, \hat{\nu})$. By the density argument it suffices to verify (2.13) for each $h$ of the form $h(x, s)=g(x) v_{0}(s)$ where $g \in C(\bar{\Omega})$ and $v_{0} \in \mathcal{R}$.

\subsubsection{Some special subsets}

We say that $(\sigma, \hat{\nu}) \in \mathcal{D} \mathcal{M}_{\mathcal{R}}^{p}\left(\Omega ; \mathbb{R}^{m \times n}\right)$ is homogeneous if $x \mapsto \hat{\nu}_{x}$ is constant. This implies that $\sigma$ is absolutely continuous with respect to the Lebesgue measure with a constant density $d_{\sigma}$. See formula (2.17) below.

The central question which we are about to answer in this contribution is which $(\sigma, \hat{\nu}) \in \mathcal{D M}_{\mathcal{R}}^{p}\left(\Omega ; \mathbb{R}^{m \times n}\right)$ are generated by gradients, i.e., by $y_{k}:=\nabla u_{k}$, for $\left\{u_{k}\right\}_{k \in \mathbb{N}} \subset W^{1, p}\left(\Omega ; \mathbb{R}^{m}\right)$ bounded. We denote the set of DiPerna-Majda measures from $\mathcal{D} \mathcal{M}_{\mathcal{R}}^{p}\left(\Omega ; \mathbb{R}^{m \times n}\right)$ which are generated by gradients $\mathcal{G} \mathcal{D} \mathcal{M}_{\mathcal{R}}^{p}\left(\Omega ; \mathbb{R}^{m \times n}\right)$.

\subsubsection{Nonconcentrating modifications}

Let us recall that for any $(\sigma, \hat{\nu}) \in \mathcal{D} \mathcal{M}_{\mathcal{R}}^{p}\left(\Omega ; \mathbb{R}^{m \times n}\right)$ there is precisely one $\left(\sigma^{\circ}, \hat{\nu}^{\circ}\right) \in \mathcal{D} \mathcal{M}_{\mathcal{R}}^{p}\left(\Omega ; \mathbb{R}^{m \times n}\right)$ such that

$$
\int_{\Omega} \int_{\mathbb{R}^{m \times n}} v_{0}(s) \hat{\nu}_{x}(\mathrm{~d} s) g(x) \sigma(\mathrm{d} x)=\int_{\bar{\Omega}} \int_{\mathbb{R}^{m \times n}} v_{0}(s) \hat{\nu}_{x}^{\circ}(\mathrm{d} s) g(x) \sigma^{\circ}(\mathrm{d} x)
$$

for any $v_{0} \in C_{0}\left(\mathbb{R}^{m \times n}\right)$ and any $g \in C(\bar{\Omega})$ and $\left(\sigma^{\circ}, \hat{\nu}^{\circ}\right)$ is attainable by a sequence $\left\{y_{k}\right\}_{k \in \mathbb{N}}$ such that the set $\left\{\left|y_{k}\right|^{p} ; k \in \mathbb{N}\right\}$ is relatively weakly compact in $L^{1}(\Omega)$; see $[25,36]$ for details. We call $\left(\sigma^{\circ}, \hat{\nu}^{\circ}\right)$ the nonconcentrating modification of $(\sigma, \hat{\nu})$. In general we call $(\sigma, \hat{\nu}) \in \mathcal{D} \mathcal{M}_{\mathcal{R}}^{p}\left(\Omega ; \mathbb{R}^{m \times n}\right)$ nonconcentrating if

$$
\int_{\bar{\Omega}} \int_{\beta_{\mathcal{R}} \mathbb{R}^{m \times n} \backslash \mathbb{R}^{m \times n}} \hat{\nu}_{x}(\mathrm{~d} s) \sigma(\mathrm{d} x)=0,
$$

and property (2.15) completely describes all measures $(\sigma, \hat{\nu})$ which can be generated by such a sequence $\left\{y_{k}\right\}_{k \in \mathbb{N}}$ that $\left\{\left|y_{k}\right|^{p}\right\}_{k \in \mathbb{N}}$ is relatively weakly compact in $L^{1}(\Omega)$. In particular if $\left(\sigma^{\circ}, \hat{\nu}^{\circ}\right)$ the nonconcentrating modification 
of $(\sigma, \hat{\nu})$ then $\hat{\nu}_{x}^{0}\left(\beta_{\mathcal{R}} \mathbb{R}^{m \times n} \backslash \mathbb{R}^{m \times n}\right)=0$ for $\sigma^{0}$ almost all $x$. Note also that $\sigma^{0}$ is absolutely continuous with respect to the Lebesgue measure (because the generating sequence is relatively weakly compact in $L^{1}(\Omega)$ ).

We wish to emphasize the following fact: if $\left\{y_{k}\right\} \in L^{p}\left(\Omega ; \mathbb{R}^{m \times n}\right)$ generates $(\sigma, \hat{\nu}) \in \mathcal{D} \mathcal{M}_{\mathcal{R}}^{p}\left(\Omega ; \mathbb{R}^{m \times n}\right)$ and $\sigma$ is absolutely continuous with respect to the Lebesgue measure it generally does not mean that $\left\{\left|y_{k}\right|^{p}\right\}$ is weakly relatively compact in $L^{1}(\Omega)$. Simple examples can be found e.g. in $[26,36]$.

The following lemma recalls some facts about of the $p$-nonconcentrating modification. Proofs can be found in [25], Lemma 1, Theorems 1, 2 and [36], Proposition 3.2.17.

Lemma 2.3. Let $(\sigma, \hat{\nu}) \in \mathcal{D} \mathcal{M}_{\mathcal{R}}^{p}\left(\Omega ; \mathbb{R}^{m \times n}\right)$ and let $\left(\sigma^{\circ}, \hat{\nu}^{\circ}\right) \in \mathcal{D} \mathcal{M}_{\mathcal{R}}^{p}\left(\Omega ; \mathbb{R}^{m \times n}\right)$ be its p-nonconcentrating modification. Then for almost all $x \in \Omega$

$$
d_{\sigma^{\circ}}(x)=\left(\int_{\mathbb{R}^{m \times n}} \hat{\nu}_{x}(\mathrm{~d} s)\right) d_{\sigma}(x)
$$

and

$$
\hat{\nu}_{x}^{\circ}(\mathrm{d} s)=\frac{\left[\left.\hat{\nu}_{x}\right|_{\mathbb{R}^{m \times n}}\right](\mathrm{d} s)}{\int_{\mathbb{R}^{m \times n}} \hat{\nu}_{x}(\mathrm{~d} s)},
$$

where $d_{\sigma^{\circ}}$ and $d_{\sigma}$ are densities (with respect to the Lebesgue measure) of $\sigma^{\circ}$ and $\sigma$, respectively.

Having a sequence bounded in $L^{p}\left(\Omega ; \mathbb{R}^{m \times n}\right)$ generating a DiPerna-Majda measure $(\sigma, \hat{\nu}) \in \mathcal{D} \mathcal{M}_{\mathcal{R}}^{p}\left(\Omega ; \mathbb{R}^{m \times n}\right)$ it also generates an $L^{p}$-Young measure $\nu \in \mathcal{Y}^{p}\left(\Omega ; \mathbb{R}^{m \times n}\right)$. It easily follows from [36], Theorem 3.2.13, that

$$
\nu_{x}(\mathrm{~d} s)=d_{\sigma^{\circ}}(x) \frac{\hat{\nu}_{x}^{\circ}(\mathrm{d} s)}{1+|s|^{p}} \quad \text { for a.a. } x \in \Omega .
$$

This means that for every $v \in \Upsilon_{\mathcal{R}}^{p}\left(\mathbb{R}^{m \times n}\right)$ (defined by (2.9)) we have

$$
\int_{\mathbb{R}^{m \times n}} v(s) \nu_{x}(\mathrm{~d} s)=d_{\sigma^{0}}(x) \int_{\beta_{\mathcal{R}^{m} \mathbb{R}^{m \times n}}} \frac{v(s)}{1+|s|^{p}} \hat{\nu}_{x}^{0}(\mathrm{~d} s)=d_{\sigma^{0}}(x) \int_{\mathbb{R}^{m \times n}} \frac{v(s)}{1+|s|^{p}} \hat{\nu}_{x}^{0}(\mathrm{~d} s) .
$$

As pointed out in [25], Remark 2, for almost all $x \in \Omega$

$$
d_{\sigma}(x)=\left(\int_{\mathbb{R}^{m \times n}} \frac{\hat{\nu}_{x}(\mathrm{~d} s)}{1+|s|^{p}}\right)^{-1}
$$

Observe that (2.14) can be improved to

$$
\int_{\Omega} \int_{\mathbb{R}^{m \times n}} v_{0}(s) \hat{\nu}_{x}(\mathrm{~d} s) g(x) \sigma(\mathrm{d} x)=\int_{\bar{\Omega}} \int_{\mathbb{R}^{m \times n}} v_{0}(s) \hat{\nu}_{x}^{\circ}(\mathrm{d} s) g(x) \sigma^{\circ}(\mathrm{d} x)
$$

for any $v_{0} \in \mathcal{R}$ and any $g \in C(\bar{\Omega})$. Indeed, for any $j \in N$ we define $a_{j} \in C_{0}\left(\mathbb{R}^{m}\right)$ such that $0 \leq a_{j} \leq 1, a_{j}(s)=1$ if $|s| \leq j$. Then $v_{0} a_{j}$ is admissible for (2.14) and the Lebesgue dominated convergence theorem for $j \rightarrow \infty$ applied to both sides in (2.14) implies (2.18). There is a one-to-one correspondence between nonconcentrating DiPerna-Majda measures and Young measures; cf. [36]. In particular (see (2.16), (2.18)) we deduce that for almost all $x \in \Omega$

$$
\int_{\mathbb{R}^{m \times n}} v(s) \nu_{x}(\mathrm{~d} s)=d_{\sigma}(x) \int_{\mathbb{R}^{m \times n}} v_{0}(s) \hat{\nu}_{x}(\mathrm{~d} s)
$$

whenever $v \in \Upsilon_{\mathcal{R}}^{p}\left(\mathbb{R}^{m \times n}\right)$. This finally yields that $\forall g \in C(\bar{\Omega}) \forall v_{0} \in \mathcal{R}$ :

$$
\lim _{k \rightarrow \infty} \int_{\Omega} g(x) v\left(y_{k}(x)\right) \mathrm{d} x=\int_{\Omega} \int_{\mathbb{R}^{m \times n}} v(s) \nu_{x}(\mathrm{~d} s) g(x) \mathrm{d} x+\int_{\bar{\Omega}} \int_{\beta_{\mathcal{R}} \mathbb{R}^{m \times n} \backslash \mathbb{R}^{m \times n}} v_{0}(s) \hat{\nu}_{x}(\mathrm{~d} s) g(x) \sigma(\mathrm{d} x),
$$


where $\nu \in \mathcal{Y}^{p}\left(\Omega ; \mathbb{R}^{m \times n}\right)$ and $(\sigma, \hat{\nu}) \in \mathcal{D M}_{\mathcal{R}}^{p}\left(\Omega ; \mathbb{R}^{m \times n}\right)$ are Young and DiPerna-Majda measures generated by $\left\{y_{k}\right\}_{k \in \mathbb{N}}$, respectively.

\subsubsection{Characterization of DiPerna-Majda measures}

The following proposition from [25] explicitly characterizes elements of $\mathcal{D} \mathcal{M}_{\mathcal{R}}^{p}\left(\Omega ; \mathbb{R}^{m \times n}\right)$.

Proposition 2.4. Let $\Omega \subset \mathbb{R}^{n}$ be a bounded open domain such that $|\partial \Omega|=0, \mathcal{R}$ be a separable complete subring of the ring of all continuous bounded functions on $\mathbb{R}^{m \times n}$ and $(\sigma, \hat{\nu}) \in \operatorname{rca}(\bar{\Omega}) \times L_{\mathrm{w}}^{\infty}\left(\bar{\Omega}, \sigma ; \operatorname{rca}\left(\beta_{\mathcal{R}} \mathbb{R}^{m \times n}\right)\right)$ and $1 \leq p<+\infty$. Then the following two statements are equivalent with each other:

(i) the pair $(\sigma, \hat{\nu})$ is the DiPerna-Majda measure, i.e. $(\sigma, \hat{\nu}) \in \mathcal{D M}_{\mathcal{R}}^{p}\left(\Omega ; \mathbb{R}^{m \times n}\right)$;

(ii) the following properties are satisfied simultaneously:

(1) $\sigma$ is positive;

(2) $\sigma_{\hat{\nu}} \in \operatorname{rca}(\bar{\Omega})$ defined by $\sigma_{\hat{\nu}}(\mathrm{d} x)=\left(\int_{\mathbb{R}^{m \times n}} \hat{\nu}_{x}(\mathrm{~d} s)\right) \sigma(\mathrm{d} x)$ is absolutely continuous with respect to the Lebesgue measure ( $d_{\sigma_{\hat{\nu}}}$ will denote its density);

(3) for a.a. $x \in \Omega$ it holds

$$
\int_{\mathbb{R}^{m \times n}} \hat{\nu}_{x}(\mathrm{~d} s)>0, \quad d_{\sigma_{\hat{\nu}}}(x)=\left(\int_{\mathbb{R}^{m \times n}} \frac{\hat{\nu}_{x}(\mathrm{~d} s)}{1+|s|^{p}}\right)^{-1} \int_{\mathbb{R}^{m \times n}} \hat{\nu}_{x}(\mathrm{~d} s) ;
$$

(4) for $\sigma$-a.a. $x \in \bar{\Omega}$ it holds

$$
\hat{\nu}_{x} \geq 0, \quad \int_{\beta_{\mathcal{R}} \mathbb{R}^{m \times n}} \hat{\nu}_{x}(\mathrm{~d} s)=1 .
$$

Remark 2.5. As pointed out to us by M. Hušek and T. Roubíček having a metrizable compactification of $\mathbb{R}^{m \times n}$ we can construct a finer one as follows

Consider a metrizable compactification $\beta_{\mathcal{R}} \mathbb{R}^{m \times n}$ of $\mathbb{R}^{m \times n}$ and the corresponding separable complete closed ring $\mathcal{R}$ with its dense subset $\left\{v_{k}\right\}_{k \in \mathbb{N}}$. We take a bounded continuous function $\psi: \mathbb{R}^{m \times n} \rightarrow \mathbb{R}, \psi \notin \mathcal{R}$ and take a closure (in the Chebyshev norm) of $\left\{\psi^{j}\right\}_{j \in \mathbb{N} \cup\{0\}} \cup\left\{\psi^{j} v_{k}\right\}_{k \in \mathbb{N}}^{j \in \mathbb{N} \cup\{0\}}$. As $\left\{\psi^{j}\right\} \cup\left\{\psi^{j} v_{k}\right\}$ is again countable the corresponding compactification is metrizable but strictly finer than $\beta_{\mathcal{R}} \mathbb{R}^{m \times n}$.

We will also use the following result, whose proof can be found in several places in various contexts (see [25], Lem. 1, Ths. 1, 2 [36], Prop. 3.2.17), [2], Proposition 4.1, part (iii) and [19], Lemma 3.1, part (ii).

Lemma 2.6. Let $\Omega \subset \mathbb{R}^{n}$ be a bounded open domain such that $|\partial \Omega|=0, \mathcal{R}$ be a separable complete subring of the ring of all continuous bounded functions on $\mathbb{R}^{m \times n}$ and $(\sigma, \hat{\nu}) \in \mathcal{D} \mathcal{M}_{\mathcal{R}}^{p}\left(\Omega ; \mathbb{R}^{m \times n}\right)$. Then for $\sigma_{s^{-}}$almost all $x \in \bar{\Omega}$ we have

$$
\hat{\nu}_{x}\left(\mathbb{R}^{m \times n}\right)=0 .
$$

\subsection{The results statement}

Our main results can be summarized to the following four theorems. The first one explicitly characterizes DiPerna-Majda measures generated by gradients of maps with the same trace. 
Theorem 2.7. Let $\Omega \subset \mathbb{R}^{n}$ be a bounded domain with the extension property in $W^{1, p}, 1<p<+\infty$ and $(\sigma, \hat{\nu}) \in \mathcal{D} \mathcal{M}_{\mathcal{R}}^{p}\left(\Omega ; \mathbb{R}^{m \times n}\right)$. Then then there is a bounded sequence $\left\{u_{k}\right\}_{k \in \mathbb{N}} \subset W^{1, p}\left(\Omega ; \mathbb{R}^{m}\right)$ such that $u_{k}=u_{j}$ on $\partial \Omega$ for any $j, k \in \mathbb{N}$ and $\left\{\nabla u_{k}\right\}_{k \in \mathbb{N}}$ generates $(\sigma, \hat{\nu})$ if and only if the following three conditions hold

$$
\exists u \in W^{1, p}\left(\Omega ; \mathbb{R}^{m}\right): \text { for a.a. } x \in \Omega: \nabla u(x)=d_{\sigma}(x) \int_{\beta_{\mathcal{R}} \mathbb{R}^{m \times n}} \frac{s}{1+|s|^{p}} \hat{\nu}_{x}(\mathrm{~d} s),
$$

for almost all $x \in \Omega$ and for all $v \in \Upsilon_{\mathcal{R}}^{p}\left(\mathbb{R}^{m \times n}\right)$ the following inequality is fulfilled

$$
Q v(\nabla u(x)) \leq d_{\sigma}(x) \int_{\beta_{\mathcal{R}} \mathbb{R}^{m \times n}} \frac{v(s)}{1+|s|^{p}} \hat{\nu}_{x}(\mathrm{~d} s),
$$

for $\sigma$-almost all $x \in \bar{\Omega}$ and all $v \in \Upsilon_{\mathcal{R}}^{p}\left(\mathbb{R}^{m \times n}\right)$ with $Q v>-\infty$ it holds that

$$
0 \leq \int_{\beta_{\mathcal{R}} \mathbb{R}^{m \times n} \backslash \mathbb{R}^{m \times n}} \frac{v(s)}{1+|s|^{p}} \hat{\nu}_{x}(\mathrm{~d} s) .
$$

Our next theorem addresses an arbitrary domain and DiPerna-Majda measures generated by gradients of maps with possibly different traces.

Theorem 2.8. Let $\Omega$ be an arbitrary bounded domain such that $|\partial \Omega|=0,1<p<+\infty$ and $(\sigma, \hat{\nu}) \in$ $\mathcal{G D} \mathcal{M}_{\mathcal{R}}^{p}\left(\Omega ; \mathbb{R}^{m \times n}\right)$ be generated by $\left\{\nabla u_{k}\right\}_{k \in \mathbb{N}}$ such that $w-\lim _{k \rightarrow \infty} u_{k}=u$ in $W^{1, p}\left(\Omega ; \mathbb{R}^{m}\right)$. Then the conditions (2.21), (2.22) hold, and (2.23) is satisfied for $\sigma$-a.a. $x \in \Omega$.

Condition (2.23) does not hold at the boundary of $\Omega$, in general. For otherwise, consider a bounded sequence $\left\{u_{k}\right\}_{k \in \mathbb{N}} \subset W^{1, p}\left(\Omega ; \mathbb{R}^{m}\right)$ converging weakly to $u \in W^{1, p}\left(\Omega ; \mathbb{R}^{m}\right)$. Let further $\left\{\nabla u_{k}\right\}$ generate a gradient Young measure $\nu \in \mathcal{Y}^{p}\left(\Omega ; \mathbb{R}^{m \times n}\right)$ and a DiPerna-Majda measure $(\sigma, \hat{\nu}) \in \mathcal{G D}_{\mathcal{R}}^{p}\left(\Omega ; \mathbb{R}^{m \times n}\right)$. The characterization of gradient Young measures by Kinderlehrer and Pedregal [22] implies that for $0 \leq g \in C(\bar{\Omega})$

$$
\int_{\Omega} g(x) v(\nabla u(x)) \mathrm{d} x \leq \int_{\Omega} \int_{\mathbb{R}^{m \times n}} v(s) g(x) \nu_{x}(\mathrm{~d} s) \mathrm{d} x
$$

for any $v \in \Upsilon_{\mathcal{R}}^{p}\left(\mathbb{R}^{m \times n}\right)$ and quasiconvex. If (2.23) always held for $\sigma$-a.a. $x \in \bar{\Omega}$, we would obtain

$$
\int_{\Omega} g(x) v(\nabla u(x)) \mathrm{d} x \leq \int_{\Omega} \int_{\mathbb{R}^{m \times n}} g(x) v(s) \nu_{x}(\mathrm{~d} s) \mathrm{d} x+\int_{\bar{\Omega}} \int_{\beta_{\mathcal{R}} \mathbb{R}^{m \times n} \backslash \mathbb{R}^{m \times n}} g(x) \frac{v(s)}{1+|s|^{p}} \hat{\nu}_{x}(\mathrm{~d} s) \sigma(\mathrm{d} x) .
$$

However, by (2.18) the right-hand side equals $\lim _{k \rightarrow \infty} \int_{\Omega} g(x) v\left(\nabla u_{k}(x)\right) \mathrm{d} x$ and thus

$$
\int_{\Omega} g(x) v(\nabla u(x)) \mathrm{d} x \leq \lim _{k \rightarrow \infty} \int_{\Omega} g(x) v\left(\nabla u_{k}(x)\right) \mathrm{d} x .
$$

On the other hand, there are examples that $(2.26)$ does not hold if $v(s)=\operatorname{det} s$ and $g=1 ; c f .[6,8]$.

Nevertheless, our Theorem 2.8 illustrates the fact that the failure of sequential weak lower semicontinuity only relates to the behavior of $\left\{\nabla u_{k}\right\}$ near the boundary of $\Omega$. Some other related results can be found in the paper [15] and references therein.

As a corollary we obtain the following variants of theorems by Meyers [30] and Acerbi and Fusco (see e.g. $[1,18,28])$. 
Theorem 2.9. Let $0 \leq g \in C(\bar{\Omega}), v \in C\left(\mathbb{R}^{m \times n}\right),|v| \leq C\left(1+|\cdot|{ }^{p}\right), C>0$, quasiconvex, and $1<p<+\infty$. Let further $\left\{u_{k}\right\} \subset W^{1, p}\left(\Omega ; \mathbb{R}^{m}\right), u_{k} \rightarrow u$ weakly in $W^{1, p}\left(\Omega ; \mathbb{R}^{m}\right)$ and at least one of the following conditions be satisfied:

(i) for any subsequence of $\left\{u_{k}\right\}$ (not relabeled) such that $1+\left|\nabla u_{k}\right|^{p} \rightarrow \sigma$ weakly* in $\operatorname{rca}(\bar{\Omega})$ it holds $\sigma(\partial \Omega)=0$

(ii) $\lim _{|s| \rightarrow \infty} \frac{v^{-}(s)}{1+|s|^{p}}=0$ where $v^{-}:=\max \{0,-v\}$,

(iii) $u_{k}=u$ on $\partial \Omega$ for any $k \in \mathbb{N}$ and $\Omega$ is Lipschitz.

Then $I(u) \leq \liminf _{k \rightarrow \infty} I\left(u_{k}\right)$, where

$$
I(u)=\int_{\Omega} g(x) v(\nabla u(x)) \mathrm{d} x
$$

\section{Remark 2.1.}

(i) If $v \geq 0$ then the assumption (ii) in Theorem 2.9 is satisfied and we retrieve the variant of Acerbi and Fusco theorem (it deals with nonnegative functions). On the other hand, in Acerbi Fusco's theorem one can relax the continuity assumptions on $g$ and even consider Caratheodory functions instead of $(x, s) \mapsto g(x) v(s)$. Therefore our theorem can be considered as a variant of Acerbi Fusco's theorem which deals with some class of continuous functions where the nonnegativity assumptions can be relaxed. To our best knowledge such an extension is missing in the literature.

(ii) In fact, the assertion in the case of (iii) in Theorem 2.9 can be deduced from the result by Meyer [30], Theorems 4 and 5 . The use of Meyers' theorem would allow for simpler but less constructive proofs of necessity in our Theorems 2.7 and 2.8 .

(iii) The condition (ii) in the theorem is satisfied if, for example, $v^{-} \leq C\left(1+|\cdot|^{q}\right)$ for some $1 \leq q<p$ in which case $-C\left(1+|s|^{q}\right) \leq v(s) \leq C\left(1+|s|^{p}\right), C>0$. This result can be found e.g. in [8].

(iv) Using the formulae (2.12) one can obtain a more general variant of the above theorem. Here we present its simplest possible formulation illustrating our result.

Some other applications of our results to the lower semicontinuity theory and their links with the results by Acerbi, Fusco and Meyers will be discussed in our forthcoming paper [20].

Our next theorem characterizes sequential weak lower semicontinuity.

Theorem 2.10. Let $0 \leq g \in C(\bar{\Omega}), v \in C\left(\mathbb{R}^{m \times n}\right),|v| \leq C\left(1+|\cdot|{ }^{p}\right), C>0$, quasiconvex, and $1<p<+\infty$. Then the functional I defined by (2.27) is sequentially weakly lower semicontinuous in $W^{1, p}\left(\Omega ; \mathbb{R}^{m}\right)$ if and only if for any bounded sequence $\left\{w_{k}\right\} \subset W^{1, p}\left(\Omega ; \mathbb{R}^{m}\right)$ such that $\nabla w_{k} \rightarrow 0$ in measure we have $\liminf _{k \rightarrow \infty} I\left(w_{k}\right) \geq I(0)$.

\section{NECESSARY CONDITIONS}

This section is devoted to the analysis of necessary conditions on the measure $(\sigma, \hat{\nu}) \in \mathcal{D M}_{\mathcal{R}}^{p}\left(\Omega ; \mathbb{R}^{m \times n}\right)$ to be generated by gradients. We start with an easy lemma whose proof is left to the reader.

Lemma 3.1. Let $M \subset \mathbb{R}^{n}$ be a bounded Borel measurable set and $\sigma, \gamma \in \mathrm{rca}(M)$ be nonnegative and such that for any nonnegative function $g \in C(M)$ we have $\int_{M} g(x) \sigma(\mathrm{d} x) \geq \int_{M} g(x) \gamma(\mathrm{d} x)$. Then $\sigma(A) \geq \gamma(A)$ for any measurable set $A \subset M$.

The following lemma shows in what cases the restriction of a DiPerna-Majda measure to a given subdomain $\omega \subseteq \Omega$ can be generated by its generating sequence restricted to $\omega$. 
Lemma 3.2. Let $(\sigma, \hat{\nu}) \in \mathcal{D} \mathcal{M}_{\mathcal{R}}^{p}\left(\Omega ; \mathbb{R}^{m \times n}\right)$ and an open domain $\omega \subseteq \Omega$ be such that $\sigma(\partial \omega)=0$. Let $\left\{y_{k}\right\}_{k \in \mathbb{N}}$ generate $(\sigma, \hat{\nu})$ in the sense $(2.8)$. Then for all $v_{0} \in \mathcal{R}$ and all $g \in C(\bar{\Omega})$

$$
\lim _{k \rightarrow \infty} \int_{\Omega} v\left(y_{k}\right) g(x) \chi_{\omega}(x) \mathrm{d} x=\int_{\Omega} \int_{\beta_{\mathcal{R}} \mathbb{R}^{m}} v_{0}(s) \hat{\nu}_{x}(\mathrm{~d} s) g(x) \chi_{\omega}(x) \sigma(\mathrm{d} x),
$$

where $\chi_{\omega}$ is the characteristic function of $\omega$ in $\Omega$.

Proof. Let $\eta \in C_{0}\left(\mathbb{R}^{n}\right)$ be supported in $\omega$ (so that $\eta \in C_{0}(\omega)$ ). We may choose a subsequence (denoted by the same expression) such that the restrictions of $y_{k}$ to $\omega,\left\{\left.y_{k}\right|_{\omega}\right\}_{k \in \mathbb{N}}$, generate the measure $(\tau, \hat{\mu}) \in \mathcal{D} \mathcal{M}^{p}\left(\omega ; \mathbb{R}^{m \times n}\right)$. We have for any $g \in C(\bar{\Omega})$ and any $v \in \Upsilon_{\mathcal{R}}^{p}\left(\mathbb{R}^{m \times n}\right)$

$$
\lim _{k \rightarrow \infty} \int_{\Omega} v\left(y_{k}\right) g(x) \eta(x) \mathrm{d} x=\int_{\Omega} \int_{\beta_{\mathcal{R}} \mathbb{R}^{m}} v_{0}(s) \hat{\nu}_{x}(\mathrm{~d} s) g(x) \eta(x) \sigma(\mathrm{d} x)=\int_{\omega} \int_{\beta_{\mathcal{R}} \mathbb{R}^{m}} v_{0}(s) \hat{\nu}_{x}(\mathrm{~d} s) g(x) \eta(x) \sigma(\mathrm{d} x)
$$

and also

$$
\lim _{k \rightarrow \infty} \int_{\omega} v\left(y_{k}\right) g(x) \eta(x) \mathrm{d} x=\int_{\omega} \int_{\beta_{\mathcal{R}} \mathbb{R}^{m}} v_{0}(s) \hat{\mu}_{x}(\mathrm{~d} s) g(x) \eta(x) \tau(\mathrm{d} x) .
$$

We construct a sequence $\eta_{j} \in C_{0}(\omega)$ such that $0 \leq \eta_{j} \leq 1$ and $\eta_{j}(x) \rightarrow \chi_{\omega}(x)$ for every $x \in \omega$, as $j \rightarrow \infty$. Comparing the right hand sides in (3.2) and (3.3) with $\eta=\eta_{j}$, letting $j \rightarrow \infty$, and using the Lebesgue dominated convergence theorem yield

$$
\int_{\omega} \int_{\beta_{\mathcal{R}} \mathbb{R}^{m}} v_{0}(s) \hat{\nu}_{x}(\mathrm{~d} s) g(x) \sigma(\mathrm{d} x)=\int_{\omega} \int_{\beta_{\mathcal{R}} \mathbb{R}^{m}} v_{0}(s) \hat{\mu}_{x}(\mathrm{~d} s) g(x) \tau(\mathrm{d} x)=\int_{\bar{\omega}} \int_{\beta_{\mathcal{R}} \mathbb{R}^{m}} v_{0}(s) \hat{\mu}_{x}(\mathrm{~d} s) g(x) \tau(\mathrm{d} x) .
$$

The last equality holds because by Lemma $3.1 \tau$ is dominated by $\sigma$, so that $\tau(\partial \omega)=0$. As (3.4) holds for an arbitrary subsequence of $\left\{y_{k}\right\}$ such that $\left\{\left.y_{k}\right|_{\omega}\right\}_{k \in \mathbb{N}}$ generate some DiPerna-Majda measure, we see that it holds for the whole sequence $\left\{y_{k}\right\}$ generating $(\sigma, \hat{\nu})$.

The following lemma explains the diagonal procedure which will be used in the sequel.

Lemma 3.3. Let $1 \leq p<\infty$ and $A \subset L^{p}\left(\Omega, \mathbb{R}^{m \times n}\right)$ be an arbitrary bounded subset. Denote by $\operatorname{DM}_{\mathcal{R}, A}^{p}\left(\Omega ; \mathbb{R}^{m \times n}\right)$ the subset of $\operatorname{DM}_{\mathcal{R}}^{p}\left(\Omega ; \mathbb{R}^{m \times n}\right)$ consisting of all DiPerna-Majda measures that are generated by such sequences $\left\{y_{k}\right\}_{k \in \mathbb{N}}$ that $y_{k} \in A$ for every $k \in \mathbb{N}$. Then $\operatorname{DM}_{\mathcal{R}, A}^{p}\left(\Omega ; \mathbb{R}^{m \times n}\right)$ is a closed subset of $\operatorname{DM}_{\mathcal{R}}^{p}\left(\Omega ; \mathbb{R}^{m \times n}\right)$ (with respect to the weak* convergence, see (2.13)). Moreover, if $\eta^{r} \cong\left(\sigma^{r}, \hat{\nu}^{r}\right), \eta \cong(\sigma, \hat{\nu}) \in \operatorname{DM}_{\mathcal{R}, A}^{p}\left(\Omega ; \mathbb{R}^{m \times n}\right)$ are such that $\left(\sigma^{r}, \hat{\nu}^{r}\right) \stackrel{*}{\rightarrow}(\sigma, \hat{\nu})$ and $\left(\sigma^{r}, \hat{\nu}^{r}\right)$ are generated by $\left\{y_{k}^{r}\right\}_{k \in \mathbb{N}}$, then there exist sequences $\left\{r_{l}\right\}_{l \in \mathbb{N}},\left\{k_{l}\right\}_{l \in \mathbb{N}} \subset \mathbb{N}$ such that $(\sigma, \hat{\nu})$ is generated by $\left\{y_{k_{l}}^{r_{l}}\right\}_{l \in \mathbb{N}}$.

Proof. Let $\left(\sigma^{r}, \hat{\nu}^{r}\right) \in \mathcal{D} \mathcal{M}_{\mathcal{R}}^{p}\left(\Omega ; \mathbb{R}^{m \times n}\right)$ be generated by sequences $\left\{y_{k}^{r}\right\}_{k \in \mathbb{N}}$ such that $y_{k}^{r} \in A$ for every $k$ and $r$. Let $D=\left\{h_{0}^{j}\right\}_{j \in \mathbb{N}}$ be an arbitrary countable dense subset in $C\left(\bar{\Omega} \times \beta_{\mathcal{R}} \mathbb{R}^{m \times n}\right)$. For every given $l \in \mathbb{N}$ and $r \in \mathbb{N}$ we find $k=k(l, r)$ such that

$$
\left|\int_{\Omega} h^{j}\left(x, y_{k(l, r)}^{r}\right) \mathrm{d} x-\int_{\bar{\Omega} \times \beta_{\mathcal{R}} \mathbb{R}^{m \times n}} h_{0}^{j}(x, s) \eta^{r}(\mathrm{~d} s \mathrm{~d} x)\right|<\frac{1}{l}, \text { for } j=1, \ldots, l,
$$

where $h_{0}^{j}(x, s) \in C\left(\bar{\Omega} \times \beta_{\mathcal{R}} \mathbb{R}^{m \times n}\right)$ is identified with $h^{j}(x, s) /\left(1+|s|^{p}\right)$ defined on $\bar{\Omega} \times \mathbb{R}^{m \times n}$. 
For every $l \in \mathbb{N}$ we find $r=r(l)$ such that

$$
\left|\int_{\bar{\Omega} \times \beta_{\mathcal{R}} \mathbb{R}^{m \times n}} h_{0}^{j}(x, s) \eta^{r}(\mathrm{~d} s \mathrm{~d} x)-\int_{\bar{\Omega} \times \beta_{\mathcal{R}} \mathbb{R}^{m \times n}} h_{0}^{j}(x, s) \eta(\mathrm{d} s \mathrm{~d} x)\right|<\frac{1}{l}, \text { for } j=1, \ldots, l .
$$

Now it is easy to see that the sequence $\left\{v_{l}\right\}_{l \in \mathbb{N}}$ where $v_{l}=y_{k(l, r(l))}^{r(l)} \in A$ generates $\eta \cong(\sigma, \hat{\nu})$.

We are now going to show that if $(\sigma, \hat{\nu}) \in \mathcal{D M}_{\mathcal{R}}^{p}\left(\Omega ; \mathbb{R}^{m \times n}\right)$ is generated by gradients and $\sigma$ is absolutely continuous with respect to the Lebesgue measure then its generating sequence of gradients may be chosen to satisfy the uniform boundary conditions.

Lemma 3.4. Let $1<p<\infty,(\sigma, \hat{\nu}) \in \mathcal{D M}_{\mathcal{R}}^{p}\left(\Omega ; \mathbb{R}^{m \times n}\right)$ and $\sigma$ be absolutely continuous with respect to the Lebesgue measure. Assume further that $(\sigma, \hat{\nu})$ is generated by a sequence $\left\{\nabla u_{k}\right\}_{k \in \mathbb{N}}$ where $\left\{u_{k}\right\}_{k \in \mathbb{N}} \subset$ $W^{1, p}\left(\Omega ; \mathbb{R}^{m}\right)$ and $w-\lim _{k \rightarrow \infty} u_{k}=u$ in $W^{1, p}\left(\Omega ; \mathbb{R}^{m}\right)$. Then there is the sequence $\left\{h_{k}\right\}_{k \in \mathbb{N}} \subset W^{1, p}\left(\Omega, \mathbb{R}^{m \times n}\right)$ such that $\left\{h_{k}-u\right\}_{k \in \mathbb{N}} \subset W_{0}^{1, p}\left(\Omega ; \mathbb{R}^{m \times n}\right)$ and $\left\{\nabla h_{k}\right\}_{k \in \mathbb{N}}$ generates $(\sigma, \hat{\nu})$.

Proof. Let $\Omega_{j}:=\{x \in \Omega ; \operatorname{dist}(x, \partial \Omega)>1 / j\}$ and $\left\{\eta_{j}\right\}_{j \in \mathbb{N}}$ be a sequence of smooth functions defined on $\mathbb{R}^{n}$ such that for any $j \in \mathbb{N}$ we have $\eta_{j} \equiv 0$ outside $\Omega, \eta_{j} \equiv 1$ on $\Omega_{j}\left|\nabla \eta_{j}\right| \leq c j$ with $c>0$ independent of $j$ and $0 \leq \eta_{j} \leq 1$. In particular $\eta_{j}(x) \rightarrow \chi_{\Omega}(x)$ for all $x \in \Omega$.

Consider $f_{j k}=\eta_{j} u_{k}+\left(1-\eta_{j}\right) u$. Then $\left\{f_{j k}-u\right\} \subset W_{0}^{1, p}\left(\Omega ; \mathbb{R}^{m}\right)$ and $\nabla f_{j k}=\eta_{j} \nabla\left(u_{k}-u\right)+\nabla u+\left(u_{k}-u\right) \otimes \nabla \eta_{j}$. Let us fix $j$ and let $\left(\sigma^{j}, \hat{\nu}^{j}\right)$ be a DiPerna-Majda measures generated by a subsequence in $k$ of $\left\{\nabla f_{j k}\right\}_{k \in \mathbb{N}}$ denoted by the same expression. By an easy computation we have

$$
\left(1+\left|\nabla f_{j k}\right|^{p}\right) \leq C\left(1+\left|\nabla u_{k}\right|^{p}+|\nabla u|^{p}+\left|\left(u_{k}-u\right) \otimes \nabla \eta_{j}\right|^{p}\right),
$$

with some $C>0$ independent of $u, j, k, \Omega$. Therefore for any nonnegative $g \in C(\bar{\Omega})$

$$
\begin{aligned}
\int_{\bar{\Omega}} g(x) \sigma^{j}(\mathrm{~d} x) & =\lim _{k \rightarrow \infty} \int_{\Omega}\left(1+\left|\nabla f_{j k}(x)\right|^{p}\right) g(x) \mathrm{d} x \\
& \leq C \lim _{k \rightarrow \infty} \int_{\Omega}\left(1+\left|\nabla u_{k}(x)\right|^{p}+|\nabla u(x)|^{p}\right) g(x) \mathrm{d} x+C \lim _{k \rightarrow \infty} \int_{\Omega}\left|\left(u_{k}(x)-u(x)\right) \otimes \nabla \eta_{j}(x)\right|^{p} g(x) \mathrm{d} x
\end{aligned}
$$

and the first term on the right-hand side is the same as $C \int_{\Omega} g(x) \pi(\mathrm{d} x)$ where $\pi=\sigma+|\nabla u|^{p} \mathrm{~d} x$. The second term is 0 because by the assumption $u_{k} \rightarrow u$ strongly in $L^{p}\left(\Omega ; \mathbb{R}^{m}\right)$. According to Lemma 3.1 we we see that $\sigma^{j} \leq \pi$. Since $\pi$ is absolutely continuous with respect to the Lebesgue measure, so is $\sigma^{j}$. Let us denote its density by $d_{\sigma^{j}}$.

Lemma 3.2 applied to $\Omega_{j}$ and to $\Omega \backslash \bar{\Omega}_{j}$ says that for any $v \in \Upsilon_{\mathcal{R}}^{p}\left(\mathbb{R}^{m \times n}\right), g \in C(\bar{\Omega})$

$$
\begin{array}{r}
\lim _{k \rightarrow \infty} \int_{\Omega} v\left(\nabla f_{j k}(x)\right) g(x) \mathrm{d} x=\lim _{k \rightarrow \infty} \int_{\Omega} v\left(\nabla u_{k}(x)\right) \chi_{\Omega_{j}}(x) g(x) \mathrm{d} x+\lim _{k \rightarrow \infty} \int_{\Omega} v\left(\nabla f_{j k}\right) \chi_{\Omega \backslash \Omega_{j}}(x) g(x) \mathrm{d} x \\
=\int_{\Omega} \int_{\beta_{\mathcal{R}} \mathbb{R}^{m \times n}} \frac{v(s)}{1+|s|^{p}} \hat{\nu}_{x}(\mathrm{~d} s) g(x) \chi_{\Omega_{j}}(x) d_{\sigma}(x) \mathrm{d} x+\lim _{k \rightarrow \infty} \int_{\Omega} v\left(\nabla f_{j k}(x)\right) \chi_{\Omega \backslash \Omega_{j}}(x) g(x) \mathrm{d} x .
\end{array}
$$

Then

$$
\begin{aligned}
\lim _{k \rightarrow \infty}\left|\int_{\Omega} v\left(\nabla f_{j k}(x)\right) \chi_{\Omega \backslash \Omega_{j}}(x) g(x) \mathrm{d} x\right| & =\left|\int_{\Omega} \int_{\beta_{\mathcal{R}} \mathbb{R}^{m \times n}} \frac{v(s)}{1+|s|^{p}} \hat{\nu}_{x}^{j}(\mathrm{~d} s) g(x) \chi_{\Omega \backslash \Omega_{j}}(x) d_{\sigma^{j}}(x) \mathrm{d} x\right| \\
& \leq\|g\|_{C(\bar{\Omega})}\left\|v_{0}\right\|_{C\left(\beta_{\mathcal{R}} \mathbb{R}^{m \times n}\right)} \int_{\Omega} \chi_{\Omega \backslash \Omega_{j}}(x) d_{\pi}(x) \mathrm{d} x .
\end{aligned}
$$


The Lebesgue dominated convergence theorem yields

$$
\lim _{j \rightarrow \infty} \lim _{k \rightarrow \infty}\left|\int_{\Omega} v\left(\nabla f_{j k}(x)\right) \chi_{\Omega \backslash \Omega_{j}}(x) g(x) \mathrm{d} x\right|=0
$$

and finally, for $v_{0}(s)=v(s) /\left(1+|s|^{p}\right)$

$$
\begin{aligned}
\lim _{j \rightarrow \infty} \int_{\bar{\Omega}} \int_{\beta_{\mathcal{R}} \mathbb{R}^{m \times n}} v_{0}(s) \hat{\nu}_{x}^{j}(\mathrm{~d} s) g(x) \sigma^{j}(\mathrm{~d} x)= & \\
\lim _{j \rightarrow \infty} \lim _{k \rightarrow \infty} \int_{\Omega} v\left(\nabla f_{j k}(x)\right) g(x) \mathrm{d} x & =\int_{\bar{\Omega}} \int_{\beta_{\mathcal{R}} \mathbb{R}^{m \times n}} v_{0}(s) \hat{\nu}_{x}(\mathrm{~d} s) g(x) \sigma(\mathrm{d} x) .
\end{aligned}
$$

Now it suffices to apply Lemma 3.3 with $A=\left\{\nabla f_{j k} ; j, k \in \mathbb{N}\right\}$.

The following lemma shows that gradient DiPerna-Majda measures are "collected" from homogeneous ones.

Lemma 3.5. Let $(\sigma, \hat{\nu}) \in \mathcal{G} \mathcal{D} \mathcal{M}_{\mathcal{R}}^{p}\left(\Omega ; \mathbb{R}^{m \times n}\right), 1<p<+\infty$. Then for almost all $a \in \Omega$, the couple $(\pi, \hat{\mu})$, where $\hat{\mu}_{x}=\hat{\nu}_{a}$ for a.a. $x \in \Omega$ and $\pi(\mathrm{d} x)=d_{\sigma}(a) \mathrm{d} x$, is a gradient DiPerna-Majda measure, i.e. $(\pi, \hat{\mu}) \in$ $\mathcal{G} \mathcal{D} \mathcal{M}_{\mathcal{R}}^{p}\left(\Omega ; \mathbb{R}^{m \times n}\right)$ and by the formula $(2.17)$ we have

$$
\pi(\mathrm{d} x)=\left(\int_{\mathbb{R}^{m \times n}} \frac{\hat{\nu}_{a}(\mathrm{~d} s)}{1+|s|^{p}}\right)^{-1} \mathrm{~d} x .
$$

Proof. Notice that $(\pi, \hat{\mu}) \in \mathcal{D} \mathcal{M}_{\mathcal{R}}^{p}\left(\Omega ; \mathbb{R}^{m \times n}\right)$ by Proposition 2.4. Let $\left\{\nabla u_{k}\right\}$ be a generating sequence of $(\sigma, \hat{\nu}) \in \mathcal{D} \mathcal{M}_{\mathcal{R}}^{p}\left(\Omega ; \mathbb{R}^{m \times n}\right)$ with $\left\{u_{k}\right\}$ bounded in $W^{1, p}\left(\Omega ; \mathbb{R}^{m}\right)$. We look for a sequence $\left\{u_{k, j}^{a}\right\}_{k \in \mathbb{N}, j>0}$ uniformly bounded in $W^{1, p}\left(\Omega ; \mathbb{R}^{m}\right)$ such that

$$
\nabla u_{k, j}^{a}(x)=\nabla u_{k}\left(a+j^{-1} x\right), j>0, x \in \Omega
$$

We proceed similarly as in [33], Theorem 7.2 and apply Lemma 3.2 for any $\omega:=a+j^{-1} \Omega$ with $j$ large enough. First we choose $a \in \Omega$. Define $\bar{V}_{\ell}(y)=d_{\sigma}(y) \int_{\beta_{\mathcal{R}} \mathbb{R}^{m \times n}} v_{0}^{\ell}(s) \hat{\nu}_{y}(\mathrm{~d} s)$ where $\left\{v_{0}^{\ell}\right\}_{\ell \in \mathbb{N}}$ is a dense subset of $\mathcal{R}$. Then we take $a \in \Omega, a \in \mathcal{L}_{u} \cap \mathcal{L}_{d_{\sigma}} \cap_{\ell=1}^{\infty} \mathcal{L}_{V_{\ell}}$ (see Sect. 2.1) for any $\ell \in \mathbb{N}$. The set of such points has the full Lebesgue measure.

We know that $\left\{\nabla u_{k}\right\}$ is bounded in $L^{p}\left(\Omega ; \mathbb{R}^{m \times n}\right)$. Moreover, $\mathrm{w}^{*}-\lim _{k \rightarrow \infty} 1+\left|\nabla u_{k}\right|^{p}=\sigma$. In other words, for any $\xi \in C(\bar{\Omega})$

$$
\lim _{k \rightarrow \infty} \int_{\Omega} \xi(x)\left(1+\left|\nabla u_{k}(x)\right|^{p}\right) \mathrm{d} x=\int_{\bar{\Omega}} \xi(x) \sigma(\mathrm{d} x) .
$$

We take $\xi_{a, j} \in C_{0}(\Omega)$ such that

$$
0 \leq \chi_{a+j^{-1} \Omega}(x) \leq \xi_{a, j}(x) \leq \chi_{a+2 j^{-1} \Omega}(x), x \in \Omega
$$

Then for some constant $C>0$ one gets

$$
\begin{aligned}
& \limsup _{j \rightarrow \infty} \limsup _{k \rightarrow \infty} j^{n} \int_{\Omega}\left(1+\left|\nabla u_{k}(x)\right|^{p}\right) \chi_{a+j^{-1} \Omega}(x) \mathrm{d} x \leq \limsup _{j \rightarrow \infty} \limsup _{k \rightarrow \infty} j^{n} \int_{\Omega}\left(1+\left|\nabla u_{k}(x)\right|^{p}\right) \xi_{a, j}(x) \mathrm{d} x \\
= & \limsup _{j \rightarrow \infty} j^{n} \int_{\Omega} \xi_{a, j}(x) \sigma(\mathrm{d} x) \leq \limsup _{j \rightarrow \infty} j^{n} \int_{\Omega} \chi_{a+2 j^{-1} \Omega}(x) \sigma(\mathrm{d} x) \leq C d_{\sigma}(a) .
\end{aligned}
$$


This and the Lebesgue differentiation theorem in the form

$$
\lim _{j \rightarrow \infty} j^{n} \int_{a+\Omega / j}|V(x)-V(a)| \mathrm{d} x=0,
$$

whenever $V \in L^{1}(\Omega)$ and for almost all $a$ (see e.g. [12] p. 9, [16] p. 9, or [33] p. 120), give

$$
\limsup _{j \rightarrow \infty} \limsup _{k \rightarrow \infty} j^{n} \int_{\Omega}\left|\nabla u_{k}(x)\right|^{p} \chi_{a+j^{-1} \Omega}(x) \mathrm{d} x=\limsup _{j \rightarrow \infty} \limsup _{k \rightarrow \infty} \int_{\Omega}\left|\nabla u_{k}\left(a+j^{-1} x\right)\right|^{p} \mathrm{~d} x<+\infty .
$$

Suppose that $\mathrm{w}-\lim _{k \rightarrow \infty} u_{k}=u$ in $W^{1, p}\left(\Omega ; \mathbb{R}^{m}\right), u_{a}: \Omega \rightarrow \mathbb{R}^{m}$ is given by $u_{a}(x)=\nabla u(a) x$ and denote $C_{a}=|\Omega|^{-1} \int_{\Omega} u_{a}(x) \mathrm{d} x$. Take

$$
u_{k, j}^{a}(x)=j\left(u_{k}\left(a+j^{-1} x\right)-M_{a, k, j}\right),
$$

where $M_{a, k, j}$ is a constant chosen so that $\int_{\Omega} u_{k, j}^{a}(x) \mathrm{d} x=C_{a}$. By the Poincaré inequality $\left\{u_{k, j}^{a}\right\}_{k \in \mathbb{N}, j>0}$ is uniformly bounded in $W^{1, p}\left(\Omega ; \mathbb{R}^{m}\right)$.

Taking $v \in \Upsilon_{\mathcal{R}}^{p}\left(\mathbb{R}^{m \times n}\right)$ and $g \in C(\bar{\Omega})$ we have

$$
\int_{\Omega} v\left(\nabla u_{k, j}^{a}(x)\right) g(x) \mathrm{d} x=\int_{\Omega} v\left(\nabla u_{k}\left(a+j^{-1} x\right) g(x) \mathrm{d} x=j^{n} \int_{\Omega} v\left(\nabla u_{k}(y)\right) \chi_{a+j^{-1} \Omega}(y) g\left(\frac{y-a}{j^{-1}}\right) \mathrm{d} y .\right.
$$

Using Lemma 3.2 we get for all $v^{\ell}=v_{0}^{\ell}\left(1+|\cdot|^{p}\right)$ and all $g \in C(\bar{\Omega})$ that

$$
\begin{aligned}
\lim _{k \rightarrow \infty} \int_{\Omega} v^{\ell}\left(u_{k, j}^{a}(x)\right) g(x) \mathrm{d} x= & j^{n} \int_{\Omega} \bar{V}_{\ell}(y) \chi_{a+j^{-1} \Omega}(y) g\left(\frac{y-a}{j^{-1}}\right) \mathrm{d} y \\
& +j^{n} \int_{\bar{\Omega}} \int_{\beta_{\mathcal{R}} \mathbb{R}^{m \times n}} v_{0}^{\ell}(s) \hat{\nu}_{y}(\mathrm{~d} s) \chi_{a+j^{-1} \Omega}(y) g\left(\frac{y-a}{j^{-1}}\right) \sigma_{s}(\mathrm{~d} y)
\end{aligned}
$$

except a countable number of $j \in \mathbb{R}$. Passing to the limit for $j \rightarrow \infty$ we get by the Lebesgue differentiation theorem (3.7)

$$
\begin{aligned}
\lim _{j \rightarrow \infty} \lim _{k \rightarrow \infty} \int_{\Omega} v^{\ell}\left(\nabla u_{k, j}^{a}(x)\right) g(x) \mathrm{d} x & =\lim _{j \rightarrow \infty} \int_{\Omega} \bar{V}_{\ell}\left(a+j^{-1} x\right) g(x) \mathrm{d} x=\bar{V}_{\ell}(a) \int_{\Omega} g(x) \mathrm{d} x \\
& =\int_{\Omega} \int_{\beta_{\mathcal{R}} \mathbb{R}^{m \times n}} v_{0}^{\ell}(s) \hat{\nu}_{a}(\mathrm{~d} s) g(x) d_{\sigma}(a) \mathrm{d} x=\int_{\Omega} \int_{\beta_{\mathcal{R}} \mathbb{R}^{m \times n}} v_{0}^{\ell}(s) \hat{\mu}_{x}(\mathrm{~d} s) g(x) \pi(\mathrm{d} x) .
\end{aligned}
$$

Indeed, the second term on the right-hand side of (3.10) is in the absolute value bounded as follows (recall that $g, v_{0}^{\ell}$ are bounded)

$$
\lim _{j \rightarrow \infty} j^{n} \int_{\bar{\Omega}} \int_{\beta_{\mathcal{R}} \mathbb{R}^{m \times n}}\left|v_{0}^{\ell}(s) \hat{\nu}_{y}(\mathrm{~d} s) \chi_{a+j^{-1} \Omega}(y) g\left(\frac{y-a}{j^{-1}}\right)\right| \sigma_{s}(\mathrm{~d} y) \leq \lim _{j \rightarrow \infty} C j^{n} \int_{a+j^{-1} \Omega} \sigma_{s}(\mathrm{~d} y)=0
$$

with some $C>0$ because the density of $\sigma_{s}$ with respect to the Lebesgue measure is zero and we supposed that $\sigma_{s}(\{a\})=0$. The proof is finished using Lemma 3.3 where we deal with the set $A=\left\{\nabla u_{k, j}^{a} ; k, j \in \mathbb{N}\right\}$.

The following result will be useful when we deal with concentrations.

Lemma 3.6. Let $\sigma \in \operatorname{rca}(\bar{\Omega})$ and $\omega \subset \Omega$ be an arbitrary subdomain. Let us further denote for every $r \in \mathbb{R}$ the set $\omega_{r}:=\{x \in \omega: \operatorname{dist}(x, \partial \omega)>r\}$. Then $\sigma\left(\partial \omega_{r}\right) \neq 0$ for at most a countable number of $r$. 
Proof. Obviously we can assume that $\sigma$ is the nonnegative measure by substituting the total variation of $\sigma$ instead of $\sigma$. We define the function $F: \mathbb{R} \rightarrow[0, \infty)$ by the formulae

$$
F(r):=\sigma\left(\omega_{r}\right)
$$

As $F$ is nondecreasing and bounded, therefore it cannot have an infinitely many jumps (this simple fact is often used in the probability theory where one deals with the distribution function, see e.g. [7], Th. 14.1, p. 188). By the monotonicity property of the (regular) measure we have

$$
\lim _{t \rightarrow r, t<r} F(t)=\sigma\left(\bigcap_{t: t<r} \omega_{t}\right)=\sigma\left(\bar{\omega}_{r}\right) \text { and } \lim _{t \rightarrow r, t>r} F(t)=\sigma\left(\bigcup_{t: t>r} \omega_{t}\right)=\sigma\left(\omega_{r}\right) .
$$

Therefore the jump of $F$ at $r$ equals $-\sigma\left(\partial \omega_{r}\right)$ and the lemma follows.

Our next lemma gives a Jensen-like inequality characterizing behavior of $\hat{\nu}$ on the remainder $\beta_{\mathcal{R}} \mathbb{R}^{m \times n} \backslash \mathbb{R}^{m \times n}$.

Lemma 3.7. Let $(\sigma, \hat{\nu}) \in \mathcal{G} \mathcal{D} \mathcal{M}_{\mathcal{R}}^{p}\left(\Omega ; \mathbb{R}^{m \times n}\right), 1<p<+\infty$. Then for $\sigma$-almost all $x \in \Omega$

$$
\int_{\beta_{\mathcal{R}} \mathbb{R}^{m \times n} \backslash \mathbb{R}^{m \times n}} \frac{v(s)}{1+|s|^{p}} \hat{\nu}_{x}(\mathrm{~d} s) \geq 0 .
$$

for all $v \in \Upsilon_{\mathcal{R}}^{p}\left(\mathbb{R}^{m \times n}\right)$ with $Q v>-\infty$.

Proof. Let $\left\{\nabla u_{k}\right\}$ generate $(\sigma, \hat{\nu})$ and let $\left\{z_{k}\right\}$ be the sequence constructed in Lemma 2.2. Denoting $w_{k}=u_{k}-z_{k}$ for any $k \in \mathbb{N}$ we set $R_{k}=\left\{x \in \Omega ; \nabla w_{k}(x) \neq 0\right\}$. Lemma 2.2 asserts that $\left|R_{k}\right| \rightarrow 0$ as $k \rightarrow \infty$. We get from Lemma 2.1 that for any $v \in \Upsilon_{\mathcal{R}}^{p}\left(\mathbb{R}^{m \times n}\right)$ quasiconvex with $v(0)=0$ and any $g \in C(\bar{\Omega})$

$$
\begin{aligned}
& \left|\int_{\Omega} g(x) v\left(\nabla w_{k}(x)\right) \mathrm{d} x-\int_{\Omega} g(x)\left(v\left(\nabla u_{k}(x)\right)-v\left(\nabla z_{k}(x)\right)\right) \mathrm{d} x\right| \\
& \leq\|g\|_{C(\bar{\Omega})}\left(\int_{R_{k}}\left|v\left(\nabla u_{k}(x)-\nabla z_{k}(x)\right)-v\left(\nabla u_{k}(x)\right)\right| \mathrm{d} x+\int_{R_{k}}\left|v\left(\nabla z_{k}(x)\right)\right| \mathrm{d} x\right) \\
& \leq C\|g\|_{C(\bar{\Omega})} \int_{R_{k}}\left[\left(1+\left|\nabla u_{k}(x)-\nabla z_{k}(x)\right|^{p-1}+\left|\nabla u_{k}\right|^{p-1}\right)\left|\nabla z_{k}(x)\right|+\left(1+\left|\nabla z_{k}\right|^{p}\right)\right] \mathrm{d} x \\
& \leq C^{\prime}\left(\left(\int_{R_{k}}\left|\nabla z_{k}(x)\right|^{p} \mathrm{~d} x\right)^{1 / p}+\int_{R_{k}} 1+\left|\nabla z_{k}(x)\right|^{p} \mathrm{~d} x+\int_{R_{k}}\left|\nabla z_{k}(x)\right| \mathrm{d} x\right)
\end{aligned}
$$

for constants $C, C^{\prime}>0$ (which may depend also on $\sup _{k}\left\|\nabla u_{k}\right\|_{L^{p}(\Omega)}$ and $\sup _{k}\left\|\nabla z_{k}\right\|_{L^{p}(\Omega)}$ ). The last term goes to zero as $k \rightarrow \infty$ because $\left\{\left|\nabla z_{k}\right|^{p}\right\}$ is relatively weakly compact in $L^{1}(\Omega)$ and $\left|R_{k}\right| \rightarrow 0$ as $k \rightarrow \infty$. This calculation shows that for $v \in \Upsilon_{\mathcal{R}}^{p}\left(\mathbb{R}^{m \times n}\right)$ quasiconvex we can separate oscillation and concentration effects of $\left\{\nabla u_{k}\right\}$ independently of the used compactification of $\mathbb{R}^{m \times n}$. Indeed, due to (2.12) we have for any $g \in C(\bar{\Omega})$ and any $v \in \Upsilon_{\mathcal{R}}^{p}\left(\mathbb{R}^{m \times n}\right)$ quasiconvex that

$$
\lim _{k \rightarrow \infty} \int_{\Omega} v\left(\nabla w_{k}(x)\right) g(x) \mathrm{d} x=v(0) \int_{\Omega} g(x) \mathrm{d} x+\int_{\bar{\Omega}} \int_{\beta_{\mathbb{R}^{m \times n}} \backslash \mathbb{R}^{m \times n}} \frac{v(s)}{1+|s|^{p}} \hat{\nu}(\mathrm{d} s) g(x) \sigma(\mathrm{d} x) .
$$

Let $x_{0} \in \Omega$ and let $\zeta \in C_{0}^{\infty}\left(B\left(x_{0}, r\right)\right), 0 \leq \zeta \leq 1$. We have for any fixed $v \in \Upsilon_{\mathcal{R}}^{p}\left(\mathbb{R}^{m \times n}\right)$ with $Q v>-\infty$ that $|Q v(s)| \leq c\left(1+|s|^{p}\right)$, for all $s \in \mathbb{R}^{m \times n}$ with a constant $c>0$, cf. [24], Lemma 2.5. Therefore if $v \in \Upsilon_{\mathcal{R}}^{p}\left(\mathbb{R}^{m \times n}\right)$ 
with $Q v>-\infty$ we get by Lemma 2.1

$$
\begin{aligned}
& \left|B\left(x_{0}, r\right)\right| Q v(0) \leq \int_{B\left(x_{0}, r\right)} Q v\left(\nabla\left(\zeta(x) w_{k}(x)\right)\right) \mathrm{d} x=\int_{B\left(x_{0}, r\right)} Q v\left(\zeta(x) \nabla w_{k}(x)+w_{k}(x) \otimes \nabla \zeta(x)\right) \mathrm{d} x \\
\leq & \int_{B\left(x_{0}, r\right)} Q v\left(\zeta(x) \nabla w_{k}(x)\right) \mathrm{d} x+\alpha \int_{B\left(x_{0}, r\right)}\left(1+\left|\zeta(x) \nabla w_{k}(x)+w_{k}(x) \otimes \nabla \zeta(x)\right|^{p-1}\right)\left|w_{k}(x) \otimes \nabla \zeta(x)\right| \mathrm{d} x \\
+ & \alpha \int_{B\left(x_{0}, r\right)}\left(\left|\zeta(x) \nabla w_{k}(x)\right|^{p-1}\right)\left|w_{k}(x) \otimes \nabla \zeta(x)\right| \mathrm{d} x \leq \int_{B\left(x_{0}, r\right)} Q v\left(\zeta(x) \nabla w_{k}(x)\right) \mathrm{d} x \\
+ & \left.\alpha \int_{B\left(x_{0}, r\right)}\left(1+2^{p-1}\right)\left|\zeta(x) \nabla w_{k}(x)\right|^{p-1}\right)\left|w_{k}(x) \otimes \nabla \zeta(x)\right| \mathrm{d} x \\
+ & \alpha \int_{B\left(x_{0}, r\right)}\left(2^{p-1}\left|w_{k}(x) \otimes \nabla \zeta(x)\right|^{p-1}\right)\left|w_{k}(x) \otimes \nabla \zeta(x)\right| \mathrm{d} x \\
\leq & \int_{B\left(x_{0}, r\right)} Q v\left(\zeta(x) \nabla w_{k}(x)\right) \mathrm{d} x+\alpha\left(1+2^{p-1}\right)\left\|\zeta \nabla w_{k}\right\|_{L^{p}\left(\Omega ; \mathbb{R}^{m \times n}\right)}^{p-1}\left\|w_{k} \otimes \nabla \zeta\right\|_{L^{p}\left(\Omega ; \mathbb{R}^{m}\right)} \\
+ & 2^{p-1} \alpha\left\|w_{k} \otimes \nabla \zeta\right\|_{L^{p}\left(\Omega ; \mathbb{R}^{n}\right)}^{p} .
\end{aligned}
$$

Since $w_{k} \rightarrow 0$ strongly in $L^{p}\left(\Omega ; \mathbb{R}^{n}\right)$ and $\left\{\nabla w_{k}\right\}_{k \in \mathbb{N}}$ is bounded in $L^{p}\left(\Omega ; \mathbb{R}^{m \times n}\right)$ the last two terms tend to zero if $k \rightarrow \infty$. Therefore we have

$$
\left|B\left(x_{0}, r\right)\right| Q v(0) \leq \liminf _{k \rightarrow \infty} \int_{B\left(x_{0}, r\right)} Q v\left(\zeta(x) \nabla w_{k}(x)\right) \mathrm{d} x .
$$

Let us choose such $r>0$ that $\sigma\left(\partial B\left(x_{0}, r\right)\right)=0$. This is possible due to Lemma 3.6. We continue with the following estimate for a suitable subsequence of $\left\{\nabla w_{k}\right\}$ (not relabeled). Note that we use Lemma 3.2 with $\omega:=B\left(x_{0}, r\right)$.

$$
\begin{aligned}
& \lim _{k \rightarrow \infty} \int_{B\left(x_{0}, r\right)} Q v\left(\zeta(x) \nabla w_{k}(x)\right) \mathrm{d} x \leq \lim _{k \rightarrow \infty} \int_{B\left(x_{0}, r\right)} Q v\left(\nabla w_{k}(x)\right) \mathrm{d} x \\
+ & \alpha \lim _{k \rightarrow \infty} \int_{B\left(x_{0}, r\right)}(1-\zeta(x))\left(1+\zeta^{p-1}(x)\right)\left|\nabla w_{k}(x)\right|^{p} \mathrm{~d} x+\alpha \lim _{k \rightarrow \infty} \int_{B\left(x_{0}, r\right)}(1-\zeta(x))\left|\nabla w_{k}(x)\right| \mathrm{d} x \\
= & \lim _{k \rightarrow \infty} \int_{B\left(x_{0}, r\right)} Q v\left(\nabla w_{k}(x)\right) \mathrm{d} x+\alpha \int_{B\left(x_{0}, r\right)} \int_{\beta_{\mathcal{R} \mathbb{R}^{m \times n}}} \frac{|s|^{p}}{1+|s|^{p}} \hat{\nu}_{x}(\mathrm{~d} s)(1-\zeta(x))\left(1+\zeta^{p-1}(x)\right) \sigma(\mathrm{d} x) \\
+ & \alpha \int_{B\left(x_{0}, r\right)} \int_{\beta_{\mathcal{R}} \mathbb{R}^{m \times n}} \frac{|s|}{1+|s|^{p}} \hat{\nu}_{x}(\mathrm{~d} s)(1-\zeta(x)) \sigma(\mathrm{d} x) .
\end{aligned}
$$

Taking into account (3.15) and (3.16) and a sequence $\left\{\zeta_{j}\right\}_{j \in \mathbb{N}} \subset C_{0}^{\infty}\left(B\left(x_{0}, r\right)\right), 0 \leq \zeta_{j} \leq 1$ pointwise tending to $\chi_{B\left(x_{0}, r\right)} \sigma$-a.e. we have by Lebesgue's dominated convergence theorem

$$
\left|B\left(x_{0}, r\right)\right| Q v(0) \leq \lim _{k \rightarrow \infty} \int_{B\left(x_{0}, r\right)} Q v\left(\nabla w_{k}(x)\right) \mathrm{d} x .
$$

The right-hand side is not greater than

$$
\left|B\left(x_{0}, r\right)\right| Q v(0)+\int_{B\left(x_{0}, r\right)} \int_{\beta_{\mathcal{R}} \mathbb{R}^{m \times n} \backslash \mathbb{R}^{m \times n}} \frac{v(s)}{1+|s|^{p}} \hat{\nu}_{x}(\mathrm{~d} s) \sigma(\mathrm{d} x) .
$$

Indeed, we can consider a complete separable ring $\mathcal{S}$ of bounded continuous functions such that $\frac{v}{1+|\cdot|^{p}} \in \mathcal{S}$ as well as $\frac{Q v}{1+|\cdot|^{p}} \in \mathcal{S}$. The metrizable compactification $\beta_{\mathcal{S}} \mathbb{R}^{m \times n}$ may be possibly finer than $\beta_{\mathcal{R}} \mathbb{R}^{m \times n} ; c f$. Remark 2.5 
for the construction. Then we have (perhaps up to a subsequence; $c f .(3.13)$ )

$$
\lim _{k \rightarrow \infty} \int_{B\left(x_{0}, r\right)} Q v\left(\nabla w_{k}(x)\right) \mathrm{d} x=\left|B\left(x_{0}, r\right)\right| Q v(0)+\int_{B\left(x_{0}, r\right)} \int_{\beta_{\mathcal{S}} \mathbb{R}^{m \times n} \backslash \mathbb{R}^{m \times n}} \frac{Q v(s)}{1+|s|^{p}} \tilde{\nu}_{x}(\mathrm{~d} s) \sigma(\mathrm{d} x)
$$

for $(\sigma, \tilde{\nu}) \in \mathcal{G} \mathcal{D} \mathcal{M}_{\mathcal{S}}^{p}\left(\Omega ; \mathbb{R}^{m \times n}\right)$. Notice that by $(2.10) \sigma$ is independent of the used ring $\mathcal{S}$. Since $Q v \leq v$ we have

$$
\lim _{k \rightarrow \infty} \int_{B\left(x_{0}, r\right)} Q v\left(\nabla w_{k}(x)\right) \mathrm{d} x \leq\left|B\left(x_{0}, r\right)\right| Q v(0)+\int_{B\left(x_{0}, r\right)} \int_{\beta_{\mathcal{S}} \mathbb{R}^{m \times n} \backslash \mathbb{R}^{m \times n}} \frac{v(s)}{1+|s|^{p}} \tilde{\nu}_{x}(\mathrm{~d} s) \sigma(\mathrm{d} x)
$$

As $v_{0}=v /\left(1+|\cdot|^{p}\right) \in \mathcal{S}$, too, we have using $(2.19)$

$$
\begin{aligned}
& \lim _{k \rightarrow \infty} \int_{B\left(x_{0}, r\right)} v\left(\nabla u_{k}(x)\right) \mathrm{d} x=\int_{B\left(x_{0}, r\right)} \int_{\mathbb{R}^{m \times n}} v(s) \nu_{x}(\mathrm{~d} s) \\
& +\int_{B\left(x_{0}, r\right)} \int_{\beta_{\mathcal{S}} \mathbb{R}^{m \times n} \backslash \mathbb{R}^{m \times n}} \frac{v(s)}{1+|s|^{p}} \tilde{\nu}_{x}(\mathrm{~d} s) \sigma(\mathrm{d} x) \\
& =\int_{B\left(x_{0}, r\right)} \int_{\mathbb{R}^{m \times n}} v(s) \nu_{x}(\mathrm{~d} s) \mathrm{d} x \\
& +\int_{B\left(x_{0}, r\right)} \int_{\beta_{\mathcal{R}} \mathbb{R}^{m \times n} \backslash \mathbb{R}^{m \times n}} \frac{v(s)}{1+|s|^{p}} \hat{\nu}_{x}(\mathrm{~d} s) \sigma(\mathrm{d} x),
\end{aligned}
$$

where $\nu \in \mathcal{Y}^{p}\left(\Omega ; \mathbb{R}^{m \times n}\right)$ is the Young measure generated by $\left\{\nabla u_{k}\right\}_{k \in \mathbb{N}}$. Therefore,

$$
\int_{B\left(x_{0}, r\right)} \int_{\beta_{\mathcal{R}} \mathbb{R}^{m \times n} \backslash \mathbb{R}^{m \times n}} \frac{v(s)}{1+|s|^{p}} \hat{\nu}_{x}(\mathrm{~d} s) \sigma(\mathrm{d} x)=\int_{B\left(x_{0}, r\right)} \int_{\beta_{\mathcal{S}} \mathbb{R}^{m \times n} \backslash \mathbb{R}^{m \times n}} \frac{v(s)}{1+|s|^{p}} \tilde{\nu}_{x}(\mathrm{~d} s) \sigma(\mathrm{d} x) .
$$

Combining (3.18) and (3.18) we arrive at (3.17).

Thus it yields

$$
0 \leq \int_{B\left(x_{0}, r\right)} \int_{\beta_{\mathcal{R}} \mathbb{R}^{m \times n} \backslash \mathbb{R}^{m \times n}} \frac{v(s)}{1+|s|^{p}} \hat{\nu}_{x}(\mathrm{~d} s) \sigma(\mathrm{d} x) .
$$

Therefore, by Lebesgue-Besicovitch differentiation theorem [12], p. 43 for any $\sigma$-Lebesgue point $x_{0}$ of $x \mapsto$ $\int_{\beta_{\mathcal{R}} \mathbb{R}^{m \times n} \backslash \mathbb{R}^{m \times n}} \frac{v(s)}{1+|s|^{p}} \hat{\nu}_{x}(\mathrm{~d} s)$ and any sequence $\left\{r_{j}\right\}_{j \in \mathbb{N}}$ such that $B\left(x_{0}, r_{j}\right) \subset \Omega, \sigma\left(\partial B\left(x_{0}, r_{j}\right)\right)=0$, and $\lim _{j \rightarrow \infty} r_{j}=$ 0 (its existence follows from Lemma 3.6) we get

$$
\begin{aligned}
0 & \leq \lim _{j \rightarrow \infty} \frac{1}{\sigma\left(B\left(x_{0}, r_{j}\right)\right)} \int_{B\left(x_{0}, r_{j}\right)} \int_{\beta_{\mathcal{R}} \mathbb{R}^{m \times n} \backslash \mathbb{R}^{m \times n}} \frac{v(s)}{1+|s|^{p}} \hat{\nu}_{x}(\mathrm{~d} s) \sigma(\mathrm{d} x) \\
& =\int_{\beta_{\mathcal{R}} \mathbb{R}^{m \times n} \backslash \mathbb{R}^{m \times n}} \frac{v(s)}{1+|s|^{p}} \hat{\nu}_{x_{0}}(\mathrm{~d} s) .
\end{aligned}
$$

We continue similarly as in [14]. The previous calculation yields the existence of a $\sigma$-null set $E_{v} \subset \Omega$ such that

$$
0 \leq \int_{\beta_{\mathcal{R}} \mathbb{R}^{m \times n} \backslash \mathbb{R}^{m \times n}} \frac{v(s)}{1+|s|^{p}} \hat{\nu}_{x}(\mathrm{~d} s)
$$

if $x \notin E_{v}$. Let $\left\{v_{0}^{k}\right\}_{k \in \mathbb{N}}$ be a dense subset of $\mathcal{R}$, so that $\left\{v^{k}\right\}_{k \in \mathbb{N}}=\left\{v_{0}^{k}\left(1+\left.|\cdot|\right|^{p}\right)\right\}_{k \in \mathbb{N}} \subset \Upsilon_{\mathcal{R}}^{p}\left(\mathbb{R}^{m \times n}\right)$. We define

$$
E=\bigcup_{k} \bigcup_{\left\{j \in \mathbb{N} ; Q\left(v^{k}+(1 / j)\left(1+|\cdot|^{p}\right)\right)>-\infty\right\}} E_{v^{k}+(1 / j)\left(1+|\cdot|^{p}\right)}
$$


Clearly $\sigma(E)=0$. Fix $x \in(\Omega \backslash E), v \in \Upsilon_{\mathcal{R}}^{p}\left(\mathbb{R}^{m \times n}\right)$ such that $Q v>-\infty$ and choose a subsequence (not relabeled) $\left\{v_{0}^{k}\right\}_{k \in \mathbb{N}}$ such that

$$
v_{0}^{k} \rightarrow v_{0} \text { in } C\left(\beta_{\mathcal{R}} \mathbb{R}^{m \times n}\right) \text { and }\left\|v_{0}^{k}-v_{0}\right\|_{C\left(\beta_{\mathcal{R}} \mathbb{R}^{m \times n}\right)}<\frac{1}{j(k)},
$$

where $j(k) \rightarrow \infty$ if $k \rightarrow \infty$. We have

$$
\begin{aligned}
v^{k}(s)+\frac{1}{j(k)}\left(1+|s|^{p}\right) & \geq v^{k}(s)+\left(1+|s|^{p}\right)\left\|v_{0}^{k}-v_{0}\right\|_{C\left(\beta_{\mathcal{R}} \mathbb{R}^{m \times n}\right)} \\
& \geq v^{k}(s)+\left|v_{0}^{k}(s)-v_{0}(s)\right|\left(1+|s|^{p}\right) \geq v(s) .
\end{aligned}
$$

Thus, $Q\left(v^{k}+\frac{1}{j(k)}\left(1+|s|^{p}\right)\right)+>-\infty$, as well, and because $x \notin E$ then $x \notin E_{v^{k}+(1 / j(k))\left(1+|\cdot|^{p}\right)}$ and

$$
\begin{aligned}
0 & \leq \lim _{k \rightarrow \infty} \int_{\beta_{\mathcal{R}} \mathbb{R}^{m \times n} \backslash \mathbb{R}^{m \times n}}\left(v_{0}^{k}(s)+\frac{1}{j(k)}\right) \hat{\nu}_{x}(\mathrm{~d} s)=\int_{\beta_{\mathcal{R}} \mathbb{R}^{m \times n} \backslash \mathbb{R}^{m \times n}} v_{0}(s) \hat{\nu}_{x}(\mathrm{~d} s) \\
& =\int_{\beta_{\mathcal{R}} \mathbb{R}^{m \times n} \backslash \mathbb{R}^{m \times n}} \frac{v(s)}{1+|s|^{p}} \hat{\nu}_{x}(\mathrm{~d} s) .
\end{aligned}
$$

We are now ready to formulate necessary conditions for a gradient DiPerna-Majda measure.

Proposition 3.8. Let $\Omega \subset \mathbb{R}^{n}$ be an arbitrary bounded domain. Let $\left\{u_{k}\right\} \subset W^{1, p}\left(\Omega ; \mathbb{R}^{m \times n}\right), 1<p<+\infty$ be bounded. Let further $\left\{\nabla u_{k}\right\}$ generate $(\sigma, \hat{\nu}) \in \mathcal{D M}_{\mathcal{R}}^{p}\left(\Omega ; \mathbb{R}^{m \times n}\right)$ Let $d_{\sigma}$ be the density of $\sigma$ with respect to the Lebesgue measure.

Then the following three conditions hold:

$$
\exists u \in W^{1, p}\left(\Omega ; \mathbb{R}^{m}\right): \nabla u(x)=d_{\sigma}(x) \int_{\beta_{\mathcal{R}} \mathbb{R}^{m \times n}} \frac{s}{1+|s|^{p}} \hat{\nu}_{x}(\mathrm{~d} s)
$$

for a.a. $x \in \Omega$,

for a.a. $x \in \Omega$ and all $v \in \Upsilon_{\mathcal{R}}^{p}\left(\mathbb{R}^{m \times n}\right)$ the following Jensen inequality is valid

$$
Q v(\nabla u(x)) \leq d_{\sigma}(x) \int_{\beta_{\mathcal{R}} \mathbb{R}^{m \times n}} \frac{v(s)}{1+|s|^{p}} \hat{\nu}_{x}(\mathrm{~d} s)
$$

and for $\sigma$-almost all $x \in \Omega$

$$
0 \leq \int_{\beta_{\mathcal{R}} \mathbb{R}^{m \times n} \backslash \mathbb{R}^{m \times n}} \frac{v(s)}{1+|s|^{p}} \hat{\nu}_{x}(\mathrm{~d} s)
$$

for all $v \in \Upsilon_{\mathcal{R}}^{p}\left(\mathbb{R}^{m \times n}\right)$ with $Q v>-\infty$.

Moreover, if $\Omega$ has extension property in $W^{1, p}$ and additionally $\left\{u_{k}-u\right\}_{k \in \mathbb{N}} \subset W_{0}^{1, p}\left(\Omega ; \mathbb{R}^{m}\right)$ then (3.20) holds for $\sigma$-almost all $x \in \bar{\Omega}$.

Proof. We start with the proof of the first part of the proposition deriving conditions (3.18), (3.19), (3.20).

(i) Suppose first that $\Omega$ is Lipschitz. As $p>1$ we assume that $\left\{u_{k}\right\}_{k}$ converges weakly to $u \in W^{1, p}\left(\Omega ; \mathbb{R}^{m \times n}\right)$. Thus for any $g \in C(\bar{\Omega})$

$$
\lim _{k \rightarrow \infty} \int_{\Omega} \nabla u_{k}(x) g(x) \mathrm{d} x=\int_{\Omega} \int_{\beta_{\mathcal{R}} \mathbb{R}^{m \times n}} \frac{s}{1+|s|^{p}} \hat{\nu}_{x}(\mathrm{~d} s) g(x) d_{\sigma}(x) \mathrm{d} x,
$$


which gives (3.18) by the density argument.

Let us take a fixed $a \in \Omega$, a Lebesgue point of $\nabla u$ and $\mathrm{d}_{\sigma}$ and denote $Y:=\nabla u(a)$. By Lemma 3.5 $\left(\pi, \hat{\mu}_{x}\right) \in \mathcal{D} \mathcal{M}_{\mathcal{R}}^{p}\left(\Omega ; \mathbb{R}^{m \times n}\right), \hat{\mu}_{x}=\hat{\nu}_{a}$ and $\pi(\mathrm{d} x)=d_{\sigma}(a) \mathrm{d} x$ is a homogeneous DiPerna-Majda measure with a generating sequence $\left\{\nabla \tilde{w}_{k}\right\}$, where $\left\{\tilde{w}_{k}\right\} \subset W^{1, p}\left(\Omega ; \mathbb{R}^{m}\right)$. Using Lemma 3.4 we can suppose that $\tilde{w}_{k}(x)=Y x$ if $x \in \partial \Omega$ and $k \in \mathbb{N}$. We have for any $v \in \Upsilon_{\mathcal{R}}^{p}\left(\mathbb{R}^{m \times n}\right)$

$$
\int_{\Omega} v\left(\nabla \tilde{w}_{k}(x)\right) \mathrm{d} x \geq|\Omega| Q v(Y) .
$$

Hence, we calculate for any $v \in \Upsilon_{\mathcal{R}}^{p}\left(\mathbb{R}^{m \times n}\right)$ with the finite quasiconvex envelope

$$
\begin{aligned}
\lim _{k \rightarrow \infty} \int_{\Omega} v\left(\tilde{w}_{k}(x)\right) \mathrm{d} x & =d_{\sigma}(a)|\Omega| \int_{\beta_{\mathcal{R}} \mathbb{R}^{m \times n}} \frac{v(s)}{1+|s|^{p}} \hat{\nu}_{a}(\mathrm{~d} s) \\
& \geq|\Omega| Q v(Y),
\end{aligned}
$$

which proves the first part of the statement for Lipschitz $\Omega$ because (3.20) follows from Lemma 3.7.

(ii) Assume now that $\Omega$ is an arbitrary bounded domain. We cover $\Omega$ by a sequence of its subdomains $\Omega_{j} \subset \Omega$ with a Lipschitz boundary such that $\operatorname{dist}\left(\Omega_{j}, \partial \Omega\right)<\frac{1}{j}$. Using Lemma 3.6 we may additionally assume that $\sigma\left(\partial \Omega_{j}\right)=0$. We use Lemma 3.2 and deduce that if $\left\{\nabla u_{k}\right\}$ generates $(\sigma, \hat{\nu})$ then the same sequence restricted to each $\Omega_{j}$ generates $(\sigma, \hat{\nu})$ restricted to $\Omega_{j}$. Therefore (3.18), (3.19), and (3.20) are satisfied on each $\Omega_{j}$ with the same $(\sigma, \hat{\nu})$ and $u$ and it remains to let $j \rightarrow+\infty$.

Now we prove the last statement in the proposition.

Let $\tilde{u}$ be an extension of $u$ to $\mathbb{R}^{n}$. Let us extend each function $u_{k}$ to $\mathbb{R}^{n}$ by plugging $\tilde{u}_{k}(x):=\tilde{u}(x)$ outside $\Omega$. Nikodym ACL Characterization Theorem (see e.g. [29], Sect. 1.1.3, Th. 2) ensures us that each $\tilde{u}_{k}$ belongs to $W^{1, p}\left(\mathbb{R}^{n}, \mathbb{R}^{m}\right)$. Let $\tilde{\Omega}$ be an arbitrary bounded domain with Lipschitz boundary such that $\bar{\Omega} \subset \tilde{\Omega}$ and let $\left(\tilde{\sigma}, \tilde{\nu}_{x}\right)$ be generated by $\left\{\nabla \tilde{u}_{k}\right\}_{k \in \mathbb{N}}$ restricted to $\tilde{\Omega}$. Decomposing for any $v \in \Upsilon_{\mathcal{R}}^{p}\left(\mathbb{R}^{m \times n}\right)$ and $g \in C(\bar{\Omega})$ :

$$
\int_{\tilde{\Omega}} v\left(\nabla \tilde{u}_{k}(x)\right) g(x) \mathrm{d} x=\int_{\tilde{\Omega} \backslash \Omega} v(\nabla \tilde{u}(x)) g(x) \mathrm{d} x+\int_{\Omega} v\left(\nabla u_{k}(x)\right) g(x) \mathrm{d} x
$$

and letting $k$ converge to $+\infty$ we observe that $\left\{\nabla \tilde{u}_{k}\right\}_{k \in \mathbb{N}}$ generates a DiPerna-Majda measure $(\tilde{\sigma}, \tilde{\nu})$ on $\tilde{\Omega}$ such that

$$
\tilde{\sigma}=\left\{\begin{array}{ccc}
\left(1+|\nabla \tilde{u}(x)|^{p}\right) \mathrm{d} x & \text { on } & \tilde{\Omega} \backslash \bar{\Omega} \\
\sigma & \text { on } & \bar{\Omega}
\end{array}, \tilde{\nu}_{x}=\left\{\begin{array}{cc}
\delta_{\nabla u(x)} & \text { if } x \in \tilde{\Omega} \backslash \bar{\Omega} \\
\nu_{x} & \text { if } x \in \bar{\Omega} .
\end{array}\right.\right.
$$

As $\tilde{\Omega}$ is a bounded domain with a Lipschitz boundary, we observe by Lemma 3.7 that (3.11) holds true for $\tilde{\sigma}$-almost all $x \in \tilde{\Omega}$. In particular it holds true for $\sigma$ almost all $x \in \bar{\Omega}$.

A remark is in order.

Remark 3.9. (i) In fact, (3.20) together with the characterization of gradient Young measures by Kinderlehrer and Pedregal [22] always imply (3.19). Namely, the characterization of gradient Young measures gives for $v$ continuous, $v(s) \leq C\left(1+|s|^{p}\right)$, that

$$
Q v(\nabla u(x)) \leq d_{\sigma}(x) \int_{\mathbb{R}^{m \times n}} \frac{v(s)}{1+|s|^{p}} \hat{\nu}_{x}(\mathrm{~d} s),
$$

for almost all $x \in \Omega$. This together with (3.20) implies (3.19).

On the other hand, if $\sigma$ is absolutely continuous with respect to the Lebesgue measure we see that (3.19) implies (3.20). To see this, decompose $\left\{u_{k}\right\}$ by means of Lemma 2.2 and observe that $\nabla w_{k} \rightarrow 0$ weakly in 
$L^{p}\left(\Omega ; \mathbb{R}^{m \times n}\right)$. Moreover, taking $v \in \Upsilon_{\mathcal{R}}^{p}\left(\mathbb{R}^{m \times n}\right)$ with $Q v>-\infty, Q v(0)=0$, we have applying (3.19) from Proposition 3.8 to $\left\{\nabla w_{k}\right\}_{k \in \mathbb{N}}$ and in view of (3.13) and Lemma 3.2 that

$$
0 \leq \int_{\beta_{\mathcal{R}} \mathbb{R}^{m \times n} \backslash \mathbb{R}^{m \times n}} \frac{Q v(s)}{1+|s|^{p}} \hat{\nu}_{x}(\mathrm{~d} s) \leq \int_{\beta_{\mathcal{R}} \mathbb{R}^{m \times n} \backslash \mathbb{R}^{m \times n}} \frac{v(s)}{1+|s|^{p}} \hat{\nu}_{x}(\mathrm{~d} s)
$$

which gives (3.20). Note that the requirement $Q v(0)=0$ does not restrict generality because we can always put $\tilde{v}=v-Q v(0)$ for $v \in \Upsilon_{\mathcal{R}}^{p}\left(\mathbb{R}^{m \times n}\right), Q v>-\infty$ and clearly

$$
\int_{\beta_{\mathcal{R}} \mathbb{R}^{m \times n} \backslash \mathbb{R}^{m \times n}} \frac{v(s)}{1+|s|^{p}} \hat{\nu}_{x}(\mathrm{~d} s)=\int_{\beta_{\mathcal{R}} \mathbb{R}^{m \times n} \backslash \mathbb{R}^{m \times n}} \frac{\tilde{v}(s)}{1+|s|^{p}} \hat{\nu}_{x}(\mathrm{~d} s) .
$$

Saying otherwise, (3.20) gives an extra condition only if $\sigma$ has a singular part.

(ii) An arbitrary bounded domain with Lipschitz boundary has the extension property in $W^{1, p}$. It is shown e.g. in [38], Section VI.3.

(iii) Condition (3.20) is analogous to the formula (5.1) in [14]. Particularly, if $\beta_{\mathcal{R}} \mathbb{R}^{m \times n}$ is the compactification by the sphere (3.20) coincides with [14], formula (5.1). As $(\sigma, \hat{\nu}) \in \mathcal{D M}_{\mathcal{R}}^{p}\left(\Omega ; \mathbb{R}^{m \times n}\right)$ must be such that $\sigma$ is nonnegative our conditions (3.19) and (3.20) imply conditions (i) and (ii) in Step 1 [14], p. 748. Note that as they use functions $g: \Omega \rightarrow \mathbb{R}$ vanishing on $\partial \Omega$ they do not need to take care about the behavior of the varifold for $x \in \partial \Omega$.

\section{Sufficient CONDitions}

This section is devoted to deriving sufficient conditions on a DiPerna-Majda measure to be generated by gradients. First, we show that DiPerna-Majda measures generated by sequences with the same affine boundary datum define homogeneous measures.

Lemma 4.1. Let $1<p<+\infty,\left\{u_{k}\right\}_{k \in \mathbb{N}} \subset W^{1, p}\left(\Omega ; \mathbb{R}^{m}\right)$ be a bounded sequence such that $u_{k}(x)-Y x \in$ $W_{0}^{1, p}\left(\Omega, \mathbb{R}^{m}\right)$ for any $k \in \mathbb{N}$, any $x \in \partial \Omega$ where $Y \in \mathbb{R}^{m \times n}$ is fixed. Let $(\sigma, \hat{\nu}) \in \mathcal{G D} \mathcal{M}_{\mathcal{R}}^{p}\left(\Omega ; \mathbb{R}^{m \times n}\right)$ be generated by $\left\{\nabla u_{k}\right\}$. Then there is a bounded sequence $\left\{w_{k}\right\} \subset W^{1, p}\left(\Omega ; \mathbb{R}^{m}\right)$ such that $\left\{u_{k}-w_{k}\right\}_{k \in \mathbb{N}} \subset W_{0}^{1, p}\left(\Omega ; \mathbb{R}^{m \times n}\right)$, $\left\{\nabla w_{k}\right\}_{k \in \mathbb{N}}$ generates $(\bar{\sigma}, \overline{\hat{\nu}}) \in \mathcal{G D} \mathcal{M}_{\mathcal{R}}^{p}\left(\Omega ; \mathbb{R}^{m \times n}\right), \bar{\sigma}$ is absolutely continuous with respect to the Lebesgue measure and its density $d_{\bar{\sigma}}(x)=\sigma(\bar{\Omega}) /|\Omega|$ for any $x \in \Omega$. Moreover, for any $v_{0} \in \mathcal{R}$ and almost all $x \in \Omega$

$$
\int_{\beta_{\mathcal{R}} \mathbb{R}^{m \times n}} v_{0}(s) \overline{\hat{\nu}}_{x}(\mathrm{~d} s)=\frac{1}{\sigma(\bar{\Omega})} \int_{\bar{\Omega}} \int_{\beta_{\mathcal{R}} \mathbb{R}^{m \times n}} v_{0}(s) \hat{\nu}_{x}(\mathrm{~d} s) \sigma(\mathrm{d} x),
$$

in particular $(\bar{\sigma}, \overline{\hat{\nu}})$ is homogeneous.

Proof. We follow the proof of [33], Theorem. 7.1. The family

$$
\mathcal{A}=\left\{x \in a+\epsilon \bar{\Omega} \subset \Omega ; a \in \Omega, \epsilon \leq j^{-1}\right\}
$$

is a covering of $\Omega$. There exists a countable collection $\left\{x \in a_{i j}+\epsilon_{i j} \bar{\Omega}\right\}, \epsilon_{i j} \leq 1 / j$ of pairwise disjoint sets and

$$
\Omega=\bigcup_{i}\left\{x \in a_{i j}+\epsilon_{i j} \bar{\Omega}\right\} \bigcup N_{j},\left|N_{j}\right|=0 .
$$


We see that $\sum_{i} \epsilon_{i j}^{n}=|\Omega| /|\Omega|=1$. We now take for $u_{Y}(x)=Y x, x \in \Omega$, the following sequence of mappings

$$
w_{k}(x)= \begin{cases}\epsilon_{i k} u_{k}\left(\frac{x-a_{i k}}{\epsilon_{i k}}\right)+u_{Y}\left(a_{i k}\right) & \text { if } x \in a_{i k}+\epsilon_{i k} \Omega \\ u_{Y}(x) & \text { otherwise. }\end{cases}
$$

Therefore, $w_{k}=u_{Y}$ on $\partial \Omega$ and for a.a. $x \in \Omega$

$$
\nabla w_{k}(x)=\nabla u_{k}\left(\frac{x-a_{i k}}{\epsilon_{i k}}\right)
$$

We have

$$
\int_{\Omega}\left|\nabla w_{k}(x)\right|^{p} \mathrm{~d} x=\sum_{i} \int_{a_{i k}+\epsilon_{i k} \Omega}\left|\nabla u_{k}\left(\frac{x-a_{i k}}{\epsilon_{i k}}\right)\right|^{p} \mathrm{~d} x=\sum_{i} \epsilon_{i k}^{n} \int_{\Omega}\left|\nabla u_{k}(x)\right|^{p} \mathrm{~d} x<C .
$$

Hence, the Poincaré inequality yields the bound on $\left\{w_{k}\right\}$ in $W^{1, p}\left(\Omega ; \mathbb{R}^{m}\right)$. Further, for any $v \in \Upsilon_{\mathcal{R}}^{p}\left(\mathbb{R}^{m \times n}\right)$ and $g \in C(\bar{\Omega})$ we get

$$
\begin{aligned}
\int_{\Omega} v\left(\nabla w_{k}(x)\right) g(x) \mathrm{d} x & =\sum_{i} \epsilon_{i k}^{n} \int_{\Omega} v\left(\nabla u_{k}(y)\right) g\left(a_{i k}+\epsilon_{i k} y\right) \mathrm{d} y=I+I I, \\
I & =\sum_{i} \epsilon_{i k}^{n} \int_{\Omega} v\left(\nabla u_{k}(y)\right)\left(g\left(a_{i k}+\epsilon_{i k} y\right)-g\left(a_{i k}+\epsilon_{i k} \bar{y}_{i k}\right)\right) \mathrm{d} y \\
I I & =\left(\frac{1}{|\Omega|} \sum_{i}|\Omega| \epsilon_{i k}^{n} g\left(a_{i k}+\epsilon_{i k} \bar{y}_{i k}\right)\right) \int_{\Omega} v\left(\nabla u_{k}(y)\right) \mathrm{d} y,
\end{aligned}
$$

where $\bar{y}_{i k} \in \bar{\Omega}$ is chosen arbitrarily. Note that $|I| \leq M_{g}\left(\frac{1}{k}\right) \int_{\Omega}\left|v\left(\nabla u_{k}(y)\right)\right| \mathrm{d} y \rightarrow 0$ as $k \rightarrow \infty$.

The second term is the Riemann sum for $\int_{\Omega} g(y) \mathrm{d} y$. Hence,

$$
\begin{aligned}
\lim _{k \rightarrow \infty} \int_{\Omega} v\left(\nabla w_{k}(x)\right) g(x) \mathrm{d} x & =\int_{\Omega} g(x) \mathrm{d} x \frac{1}{|\Omega|} \int_{\bar{\Omega}} \int_{\beta_{\mathcal{R}} \mathbb{R}^{m \times n}} v_{0}(s) \hat{\nu}_{x}(\mathrm{~d} s) \sigma(\mathrm{d} x) \\
& =\int_{\Omega} g(x) \mathrm{d} x \frac{\sigma(\bar{\Omega})}{|\Omega|} \int_{\beta_{\mathcal{R} \mathbb{R}^{m \times n}}} v_{0}(s) \overline{\hat{\nu}}_{x}(\mathrm{~d} s) \\
& =\int_{\Omega} \int_{\beta_{\mathcal{R}} \mathbb{R}^{m \times n}} v_{0}(s) \overline{\hat{\nu}}_{x}(\mathrm{~d} s) g(x) \bar{\sigma}(\mathrm{d} x) .
\end{aligned}
$$

It is well known, see e.g. [33], that the set of homogeneous $W^{1, p}$-gradient Young measures $\nu$ given for any $v \in C_{p}\left(\mathbb{R}^{m \times n}\right)$ by

$$
\int_{\mathbb{R}^{m \times n}} v(s) \nu(\mathrm{d} s)=\frac{1}{|\Omega|} \int_{\Omega} v(\nabla u(x)) \mathrm{d} x, u \in W^{1, p}\left(\Omega ; \mathbb{R}^{m}\right), u(x)=Y x, x \in \partial \Omega
$$

is convex. Let us denote it by $M_{Y}$. As Young measures generated by sequences bounded in $L^{p}\left(\Omega ; \mathbb{R}^{m \times n}\right)$ can be embedded into $\mathcal{D} \mathcal{M}_{\mathcal{R}}^{p}\left(\Omega ; \mathbb{R}^{m \times n}\right.$ ) (see [36], Rem. 3.2.16) we get that $M_{Y}$ is mapped into a subset $\hat{m}_{Y}$ of $\mathcal{D} \mathcal{M}_{\mathcal{R}}^{p}\left(\Omega ; \mathbb{R}^{m \times n}\right)$ where $(\pi, \hat{\mu}) \in \hat{m}_{Y}$ if for some $u \in W^{1, p}\left(\Omega ; \mathbb{R}^{m}\right), u(x)=Y x$ if $x \in \partial \Omega$ we have

$$
d_{\pi}=\frac{1}{|\Omega|} \int_{\Omega}\left(1+|\nabla u(x)|^{p}\right) \mathrm{d} x
$$


and for any $v \in \Upsilon_{\mathcal{R}}^{p}\left(\mathbb{R}^{m \times n}\right)$

$$
\int_{\beta_{\mathcal{R}} \mathbb{R}^{m \times n}} v_{0}(s) \hat{\mu}(\mathrm{d} s)=\frac{1}{d_{\pi}|\Omega|} \int_{\Omega} v(\nabla u(x)) \mathrm{d} x .
$$

Thus we can define $\eta_{u} \in \operatorname{rca}\left(\bar{\Omega} \times \beta_{\mathcal{R}} \mathbb{R}^{m \times n}\right)$ by

$$
\left\langle\eta_{u}, g \otimes v_{0}\right\rangle=\frac{1}{|\Omega|} \int_{\Omega} v(\nabla u(x)) \mathrm{d} x \int_{\Omega} g(y) \mathrm{d} y,
$$

where $v \in \Upsilon_{\mathcal{R}}^{p}\left(\mathbb{R}^{m \times n}\right)$ and $g \in C(\bar{\Omega})$. Here we used the fact that the linear hull of $\left\{g \otimes v_{0} ; g \in C(\bar{\Omega}), v_{0} \in \mathcal{R}\right\}$ is dense in $C\left(\bar{\Omega} \times \beta_{\mathcal{R}} \mathbb{R}^{m \times n}\right)$. We see by the inspection of $M_{Y}$ that $\eta_{u}$ is a gradient DiPerna-Majda measure from $\operatorname{DM}_{\mathcal{R}}^{p}\left(\Omega ; \mathbb{R}^{m \times n}\right)$. Namely, if $\left\{\nabla u_{k}\right\}_{k \in \mathbb{N}}$ generates $\nu$ from (4.2) then the same sequence generates $\eta_{u}$. Let us also introduce $\hat{\eta}_{u} \in \operatorname{rca}\left(\beta_{\mathcal{R}} \mathbb{R}^{m \times n}\right)$ defined for any $v_{0} \in C\left(\beta_{\mathcal{R}} \mathbb{R}^{m \times n}\right)$ by

$$
\left\langle\hat{\eta}_{u}, v_{0}\right\rangle=\left\langle\eta_{u}, 1 \otimes v_{0}\right\rangle=\int_{\Omega} v(\nabla u(x)) \mathrm{d} x
$$

Clearly as $M_{Y}$ is convex, so is

$$
\hat{M}_{Y}:=\left\{\hat{\eta}_{u} ; u \in W^{1, p}\left(\Omega ; \mathbb{R}^{m}\right), u(x)=Y x \text { on } \partial \Omega\right\} \subset \operatorname{rca}\left(\beta_{\mathcal{R}} \mathbb{R}^{m \times n}\right) .
$$

We have the following result.

Lemma 4.2. Let $1<p<+\infty$ and $(\sigma, \hat{\nu}) \in \mathcal{D} \mathcal{M}_{\mathcal{R}}^{p}\left(\Omega ; \mathbb{R}^{m \times n}\right)$ be homogeneous, i.e., $\hat{\nu}_{x}=\hat{\nu}_{y}$ for all $x, y \in \Omega$ and $\sigma$ be absolutely continuous with respect to Lebesgue's measure with the constant density

$$
d_{\sigma}=\left(\int_{\mathbb{R}^{m \times n}} \frac{\hat{\nu}(\mathrm{d} s)}{1+|s|^{p}}\right)^{-1}
$$

such that for any $v \in \Upsilon_{\mathcal{R}}^{p}\left(\mathbb{R}^{m \times n}\right)$

$$
d_{\sigma} \int_{\beta_{\mathcal{R}} \mathbb{R}^{m \times n}} \frac{v(s)}{1+|s|^{p}} \hat{\nu}(\mathrm{d} s) \geq Q v(Y),
$$

where

$$
Y=d_{\sigma} \int_{\beta_{\mathcal{R} \mathbb{R}^{m \times n}}} \frac{s}{1+|s|^{p}} \hat{\nu}(\mathrm{d} s) .
$$

Then $(\sigma, \hat{\nu})$ is a homogeneous gradient DiPerna-Majda measure. Moreover, there is a sequence $\left\{\nabla w_{k}\right\}_{k \in \mathbb{N}}$ generating $(\sigma, \hat{\nu})$ such that $\left\{w_{k}-Y x\right\}_{k \in \mathbb{N}} \subset W_{0}^{1, p}\left(\Omega, \mathbb{R}^{m}\right)$.

Proof. Multiplying (4.7) by $|\Omega|$ and defining $\xi \in \operatorname{rca}\left(\bar{\Omega} \times \beta_{\mathcal{R}} \mathbb{R}^{m \times n}\right)$ by

$$
\left\langle\xi, g \otimes v_{0}\right\rangle=\int_{\Omega} d_{\sigma} \int_{\beta_{\mathcal{R}} \mathbb{R}^{m \times n}} \frac{v(s)}{1+|s|^{p}} \hat{\nu}(\mathrm{d} s) g(x) \mathrm{d} x,
$$

for any $v \in \Upsilon_{\mathcal{R}}^{p}\left(\mathbb{R}^{m \times n}\right)$ and $g \in C(\bar{\Omega})$ we get that (4.7) is equivalent to

$$
\left\langle T_{\xi}, v_{0}\right\rangle=\left\langle\xi, 1 \otimes v_{0}\right\rangle \geq|\Omega| Q v(Y)
$$


where $T_{\xi} \in \operatorname{rca}\left(\beta_{\mathcal{R}} \mathbb{R}^{m \times n}\right)$ is defined by the relation $\left\langle T_{\xi}, v_{0}\right\rangle=\left\langle\xi, 1 \otimes v_{0}\right\rangle$. We will use the Hahn-Banach theorem to show that two subsets of $\operatorname{rca}\left(\beta_{\mathcal{R}} \mathbb{R}^{m \times n}\right): \hat{M}_{Y}$ and $\hat{T}$ where $\hat{T}$ is given by

$$
\hat{T}:=\left\{T_{\xi} ; \xi \text { given by }(4.8) \text { and satisfies }(4.9)\right\} \text {, }
$$

considered as sets of functionals on the space $C\left(\beta_{\mathcal{R}} \mathbb{R}^{m \times n}\right)$ (with the weak topology), cannot be separated by an element of $C\left(\beta_{\mathcal{R}} \mathbb{R}^{m \times n}\right)$. It is easy to see that $\hat{M}_{Y} \subset \hat{T}$. Suppose that there is $a \in \mathbb{R}$ such that for a fixed $v_{0} \in \mathcal{R}\left\langle\hat{\eta}_{u}, v_{0}\right\rangle \geq a$ for all $u \in W^{1, p}\left(\Omega ; \mathbb{R}^{m}\right), u(x)=Y x$ if $x \in \partial \Omega$. This means that $\int_{\Omega} v(\nabla u(x)) \mathrm{d} x \geq a$ for any $u \in W^{1, p}\left(\Omega ; \mathbb{R}^{m}\right), u(x)=Y x$ if $x \in \partial \Omega$ and therefore $Q v(Y)|\Omega| \geq a ; c f$. (2.2). Hence, by (4.9)

$$
\left\langle T_{\xi}, v_{0}\right\rangle=\left\langle\xi, 1 \otimes v_{0}\right\rangle \geq|\Omega| Q v(Y) \geq a
$$

As this happens for each $a$, Hahn-Banach theorem implies that $T_{\xi} \in \bar{M}_{Y}$, where the closure is in the weak* topology. As $C\left(\beta_{\mathcal{R}} \mathbb{R}^{m \times n}\right)$ is separable it follows that weak ${ }^{*}$ topology of $\operatorname{rca}\left(\beta_{\mathcal{R}} \mathbb{R}^{m \times n}\right)$ is metrizable on bounded sets. Therefore there is a sequence $\left\{u_{k}\right\} \subset W^{1, p}\left(\Omega ; \mathbb{R}^{m}\right), u_{k}(x)=Y x$ on the boundary such that $\lim _{k \rightarrow \infty}\left\langle\eta_{u_{k}}, 1 \otimes v_{0}\right\rangle=\left\langle\xi, 1 \otimes v_{0}\right\rangle$. In other words, for any $v \in \Upsilon_{\mathcal{R}}^{p}\left(\mathbb{R}^{m \times n}\right)$

$$
\lim _{k \rightarrow \infty} \int_{\Omega} v\left(\nabla u_{k}(x)\right) \mathrm{d} x=d_{\sigma}|\Omega| \int_{\beta_{\mathcal{R}} \mathbb{R}^{m \times n}} \frac{v(s)}{1+|s|^{p}} \hat{\nu}(\mathrm{d} s) .
$$

Let $(\tau, \hat{\alpha}) \in \mathcal{D} \mathcal{M}_{\mathcal{R}}^{p}\left(\Omega ; \mathbb{R}^{m \times n}\right)$ be generated by $\left\{\nabla u_{k}\right\}$ or its subsequence. Then for any $v \in \Upsilon_{\mathcal{R}}^{p}\left(\mathbb{R}^{m \times n}\right)$ and $g \in C(\bar{\Omega})$

$$
\lim _{k \rightarrow \infty} \int_{\Omega} v\left(\nabla u_{k}(x)\right) g(x) \mathrm{d} x=\int_{\bar{\Omega}} \int_{\beta_{\mathcal{R}} \mathbb{R}^{m \times n}} \frac{v(s)}{1+|s|^{p}} \hat{\alpha}_{x}(\mathrm{~d} s) g(x) \tau(\mathrm{d} x) .
$$

Now we are going to apply Lemma 4.1 to $(\tau, \hat{\alpha})$. It gives us the existence of $\left\{w_{k}\right\} \subset W^{1, p}\left(\Omega ; \mathbb{R}^{m}\right)$ with the same boundary conditions as $\left\{u_{k}-w_{k}\right\} \subset W_{0}^{1, p}\left(\Omega ; \mathbb{R}^{m \times n}\right)$ such that

$$
\lim _{k \rightarrow \infty} \int_{\Omega} v\left(\nabla w_{k}(x)\right) g(x) \mathrm{d} x=\int_{\Omega} g(x) \mathrm{d} x \frac{1}{|\Omega|} \int_{\bar{\Omega}} \int_{\beta_{\mathcal{R}} \mathbb{R}^{m \times n}} \frac{v(s)}{1+|s|^{p}} \hat{\alpha}_{x}(\mathrm{~d} s) \tau(\mathrm{d} x) .
$$

Expressing the equality (4.11) for $g=1$ by means of (4.10) and plugging it into (4.12) yields

$$
\begin{aligned}
\lim _{k \rightarrow \infty} \int_{\Omega} v\left(\nabla w_{k}(x)\right) g(x) \mathrm{d} x & =d_{\sigma} \int_{\Omega} g(x) \mathrm{d} x \int_{\beta_{\mathcal{R}} \mathbb{R}^{m \times n}} \frac{v(s)}{1+|s|^{p}} \hat{\nu}(\mathrm{d} s) \\
& =\int_{\Omega} \int_{\beta_{\mathcal{R}} \mathbb{R}^{m \times n}} \frac{v(s)}{1+|s|^{p}} \hat{\nu}(\mathrm{d} s) g(x) \sigma(\mathrm{d} x)
\end{aligned}
$$

which implies the thesis.

Lemma 4.3 (see [33], Lemma 7.9, for a more general case). Let $\Omega \subset \mathbb{R}^{n}$ be an open domain with $|\partial \Omega|=0$ and let $N \subset \Omega$ be of the zero Lebesgue measure. For $r_{k}: \Omega \backslash N \rightarrow(0,+\infty)$ and $\left\{f_{k}\right\}_{k \in \mathbb{N}} \subset L^{1}(\Omega)$ there exists a set of points $\left\{a_{i k}\right\} \subset \Omega \backslash N$ and positive numbers $\left\{\epsilon_{i k}\right\}, \epsilon_{i k} \leq r_{k}\left(a_{i k}\right)$ such that $\left\{a_{i k}+\epsilon_{i k} \bar{\Omega}\right\}$ are pairwise disjoint for each $k \in \mathbb{N}, \bar{\Omega}=\cup_{i}\left\{a_{i k}+\epsilon_{i k} \bar{\Omega}\right\} \cup N_{k}$ with $\left|N_{k}\right|=0$ and for any $j \in \mathbb{N}$ and any $g \in L^{\infty}(\Omega)$

$$
\lim _{k \rightarrow \infty} \sum_{i} f_{j}\left(a_{i k}\right) \int_{a_{i k}+\epsilon_{i k} \Omega} g(x) \mathrm{d} x=\int_{\Omega} f_{j}(x) g(x) \mathrm{d} x .
$$


Proposition 4.4. Let $(\sigma, \hat{\nu}) \in \mathcal{D M}_{\mathcal{R}}^{p}\left(\Omega ; \mathbb{R}^{m \times n}\right), 1<p<+\infty$, be such that $\sigma$ is absolutely continuous with respect to Lebesgue's measure and let $d_{\sigma}$ be its density. Let further the following two conditions hold:

$$
\exists u \in W^{1, p}\left(\Omega ; \mathbb{R}^{m}\right): \nabla u(x)=d_{\sigma}(x) \int_{\mathbb{R}^{m \times n}} \frac{s}{1+|s|^{p}} \hat{\nu}_{x}(\mathrm{~d} s),
$$

for a.a. $x \in \Omega$ and all $v \in \Upsilon_{\mathcal{R}}^{p}\left(\mathbb{R}^{m \times n}\right)$ the following inequality is valid

$$
Q v(\nabla u(x)) \leq d_{\sigma}(x) \int_{\beta_{\mathcal{R}} \mathbb{R}^{m \times n}} \frac{v(s)}{1+|s|^{p}} \hat{\nu}_{x}(\mathrm{~d} s) .
$$

Then $(\sigma, \hat{\nu})$ is generated by gradients, i.e., belongs to $\mathcal{G} \mathcal{D} \mathcal{M}_{\mathcal{R}}^{p}\left(\Omega ; \mathbb{R}^{m \times n}\right)$.

Moreover, its generating sequence, $\left\{\nabla u_{k}\right\}_{k \in \mathbb{N}}$, can be chosen in the way that $\left\{u_{k}-u\right\}_{k \in \mathbb{N}} \subset W_{0}^{1, p}\left(\Omega, \mathbb{R}^{m}\right)$.

Proof. We will divide the proof into two steps. Although step (ii) is a generalization of (i), we believe that it is instructive to look first at a simpler case.

(i) Suppose first that $u$ in (4.13) and (4.14) is zero. We are looking for a sequence $\left\{u_{k}\right\}_{k \in \mathbb{N}} \subset W^{1, p}\left(\Omega ; \mathbb{R}^{m}\right)$ satisfying

$$
\lim _{k \rightarrow \infty} \int_{\Omega} v\left(\nabla u_{k}(x)\right) g(x) \mathrm{d} x=\int_{\bar{\Omega}} \int_{\beta_{\mathcal{R}} \mathbb{R}^{m \times n}} \frac{v(s)}{1+|s|^{p}} \hat{\nu}_{x}(\mathrm{~d} s) g(x) \sigma(\mathrm{d} x)
$$

for all $g \in \Gamma$ and any $v=v_{0}\left(1+|\cdot|^{p}\right), v_{0} \in S$, where $\Gamma$ and $S$ are countable dense subsets of $C(\bar{\Omega})$ and $\mathcal{R}$.

Take $r_{k}=1 / k$ and using Lemma 4.3 find $a_{i k} \in \Omega \backslash N, \epsilon_{i k} \leq 1 / k$ such that for $v_{0} \in S$ and $g \in C(\bar{\Omega})$

$$
\lim _{k \rightarrow \infty} \sum_{i} \bar{V}\left(a_{i k}\right) \int_{a_{i k}+\epsilon_{i k} \Omega} g(x) \mathrm{d} x=\int_{\Omega} \bar{V}(x) g(x) \mathrm{d} x
$$

where

$$
\bar{V}(x)=d_{\sigma}(x) \int_{\beta_{\mathcal{R}} \mathbb{R}^{m \times n}} v_{0}(s) \hat{\nu}_{x}(\mathrm{~d} s) .
$$

The system $a_{i k}+\epsilon_{i k} \bar{\Omega}$ exhausts almost all $\Omega$. We may assume that $a_{i k} \notin N,|N|=0$, by (4.14) and by Lemma 4.2 we can assume that $\left(d_{\sigma}\left(a_{i k}\right) \mathrm{d} x, \hat{\nu}_{a_{i k}}\right)$ is a homogeneous gradient DiPerna-Majda measure living in $\mathcal{D} \mathcal{M}_{\mathcal{R}}^{p}\left(\Omega ; \mathbb{R}^{m \times n}\right)$ and we call $\left\{u_{j}^{i k}\right\}_{j \in \mathbb{N}}$ its generating sequence. Recall that $u=0$, so w- $\lim _{j \rightarrow \infty} u_{j}^{i k}=0$ in $W^{1, p}\left(\Omega ; \mathbb{R}^{m}\right)$ and by Lemma 3.4 we can even suppose that $\left\{u_{j}^{i k}\right\}_{j \in \mathbb{N}} \subset W_{0}^{1, p}\left(\Omega ; \mathbb{R}^{m \times n}\right)$ and

$$
\lim _{j \rightarrow \infty} \int_{\Omega} v\left(\nabla u_{j}^{i k}(x)\right) g(x) \mathrm{d} x=\bar{V}\left(a_{i k}\right) \int_{\Omega} g(x) \mathrm{d} x
$$

Define the sequence

$$
u_{k}(x)= \begin{cases}\epsilon_{i k} u_{j}^{i k}\left(\frac{x-a_{i k}}{\epsilon_{i k}}\right) & \text { if } x \in a_{i k}+\epsilon_{i k} \Omega \\ 0 & \text { otherwise. }\end{cases}
$$

Let $\Gamma \times S=\cup_{k} E_{k}$ with $E_{k} \subset E_{k+1}$, finite sets. For $k, i$ fixed we take $j=j(k, i)$ so large that for all $\left(g, v_{0}\right) \in E_{k}$

$$
\left|\epsilon_{i k}^{n} \int_{\Omega} g\left(a_{i k}+\epsilon_{i k} y\right) v\left(\nabla u_{j}^{i k}(y)\right) \mathrm{d} y-\bar{V}\left(a_{i k}\right) \int_{a_{i k}+\epsilon_{i k} \Omega} g(x) \mathrm{d} x\right| \leq \frac{1}{2^{i} k} .
$$


Here we exploited (4.16) written for $\tilde{g}(y)=g\left(a_{i k}+\epsilon_{i k} y\right)$ instead of $g$. Using this estimate and (4.15) we get for any $\left(g, v_{0}\right) \in \Gamma \times S$

$$
\begin{aligned}
\lim _{k \rightarrow \infty} \int_{\Omega} g(x) v\left(\nabla u_{k}(x)\right) \mathrm{d} x & =\lim _{k \rightarrow \infty} \sum_{i} \epsilon_{i k}^{n} \int_{\Omega} g\left(a_{i k}+\epsilon_{i k} y\right) v\left(\nabla u_{j}^{i k}(y)\right) \mathrm{d} y \\
& =\lim _{k \rightarrow \infty} \sum_{i} \bar{V}\left(a_{i k}\right) \int_{a_{i k}+\epsilon_{i k} \Omega} g(x) \mathrm{d} x=\int_{\Omega} \bar{V}(x) g(x) \mathrm{d} x \\
& =\int_{\Omega} \int_{\beta_{\mathcal{R}} \mathbb{R}^{m \times n}} v_{0}(s) \hat{\nu}_{x}(\mathrm{~d} s) g(x) \sigma(\mathrm{d} x)
\end{aligned}
$$

as we wish. It is clear that $u_{k}-u \in W_{0}^{1, p}\left(\Omega, \mathbb{R}^{m}\right)$ for every $k$.

(ii) If $u \neq 0$ the proof is more technical. We follow [22]. As $u \in W^{1, p}\left(\Omega ; \mathbb{R}^{m}\right)$ we take $a \in \Omega$ and for $\epsilon>0$ small enough define

$$
w_{a, \epsilon}(y)=\epsilon^{-1}[u(a+\epsilon y)-u(a)-\epsilon \nabla u(a) y] .
$$

We have that $w_{a, \epsilon} \in W^{1, p}\left(\Omega ; \mathbb{R}^{m}\right)$ and

$$
\nabla w_{a, \epsilon}(y)=\nabla u(a+\epsilon y)-\nabla u(a)
$$

Based on Reshetnyak's result (see Th. 1 in [34] for $\Omega$ being a ball, an arbitrary case follows easily from this particular one), we have that for $\varepsilon \rightarrow 0$ and a.a. $a \in \Omega$

$$
\left\|\frac{1}{\epsilon}[u(a+\epsilon y)-u(a)-\epsilon \nabla u(a) y]\right\|_{W^{1, p}(\Omega)} \rightarrow 0 .
$$

Thus, for almost all $a \in \Omega$,

$$
\lim _{\epsilon \rightarrow 0}\left\|\nabla w_{a, \epsilon}\right\|_{L^{p}\left(\Omega ; \mathbb{R}^{m \times n}\right)}=0,
$$

and by the embedding theorem we find $\infty>p^{*}>p$ such that

$$
\lim _{\epsilon \rightarrow 0}\left\|w_{a, \epsilon}\right\|_{L^{p *}\left(\Omega ; \mathbb{R}^{m}\right)}=0
$$

Let's say that this is true for all $a \in \Omega \backslash N$, where $|N|=0$. Then for $a \in \Omega \backslash N$ and any $k \in \mathbb{N}$ there is $r_{k}(a)>0$ such that if $\epsilon<r_{k}(a)$ then $a+\epsilon \Omega \subset \Omega$ and

$$
\left(\int_{\Omega}\left(\epsilon^{-1}[u(a+\epsilon y)-u(a)-\epsilon \nabla u(a) y]\right)^{p^{*}} \mathrm{~d} y\right)^{1 / p^{*}} \leq \frac{1}{k} .
$$

We are looking for a sequence $\left\{u_{k}\right\}_{k \in \mathbb{N}} \subset W^{1, p}\left(\Omega ; \mathbb{R}^{m}\right)$ satisfying

$$
\lim _{k \rightarrow \infty} \int_{\Omega} v\left(\nabla u_{k}(x)\right) g(x) \mathrm{d} x=\int_{\bar{\Omega}} \int_{\beta_{\mathcal{R}} \mathbb{R}^{m \times n}} \frac{v(s)}{1+|s|^{p}} \hat{\nu}_{x}(\mathrm{~d} s) g(x) \sigma(\mathrm{d} x)
$$

for all $g \in \Gamma$ and any $v=v_{0}\left(1+|\cdot|^{p}\right), v_{0} \in S$, where $\Gamma$ and $S$ are countable dense subsets of $C(\bar{\Omega})$ and $\mathcal{R}$.

Take $r_{k}: \Omega \backslash N \rightarrow \mathbb{R}$ and using Lemma 4.3 find $a_{i k} \in \Omega \backslash N, \epsilon_{i k} \leq r_{k}\left(a_{i k}\right)$ such that for all $v_{0} \in S$ and all $g \in C(\bar{\Omega})$

$$
\lim _{k \rightarrow \infty} \sum_{i} \bar{V}\left(a_{i k}\right) \int_{a_{i k}+\epsilon_{i k} \Omega} g(x) \mathrm{d} x=\int_{\Omega} \bar{V}(x) g(x) \mathrm{d} x
$$


and

$$
\lim _{k \rightarrow \infty} \sum_{i}\left|\bar{V}\left(a_{i k}\right)\right| \int_{a_{i k}+\epsilon_{i k} \Omega} g(x) \mathrm{d} x=\int_{\Omega}|\bar{V}(x)| g(x) \mathrm{d} x
$$

where

$$
\bar{V}(x)=d_{\sigma}(x) \int_{\beta_{\mathcal{R}} \mathbb{R}^{m \times n}} v_{0}(s) \hat{\nu}_{x}(\mathrm{~d} s) .
$$

We can assume by Lemma 4.2 that $\left(d_{\sigma}\left(a_{i k}\right) \mathrm{d} x, \hat{\nu}_{a_{i k}}\right)$ is a homogeneous gradient DiPerna-Majda measure living in $\mathcal{D} \mathcal{M}_{\mathcal{R}}^{p}\left(\Omega ; \mathbb{R}^{m \times n}\right)$ and we call $\left\{\nabla u_{j}^{i k}\right\}_{j \in \mathbb{N}}$ its generating sequence. It means that

$$
\lim _{j \rightarrow \infty} \int_{\Omega} v\left(\nabla u_{j}^{i k}(x)\right) g(x) \mathrm{d} x=\bar{V}\left(a_{i k}\right) \int_{\Omega} g(x) \mathrm{d} x .
$$

We have that

$$
\mathrm{w}-\lim _{j \rightarrow \infty} u_{j}^{i k}=L^{i k} \text { in } W^{1, p}\left(\Omega ; \mathbb{R}^{m}\right)
$$

where for almost all $x L^{i k}(x)=\nabla u\left(a_{i k}\right) x$. Let $\Omega_{\ell}=\left\{x \in \Omega\right.$; $\left.\operatorname{dist}(x, \partial \Omega) \geq \ell^{-1}\right\}$.

In view of Lemma 3.2 and (4.21) we have

$$
\lim _{j \rightarrow \infty} \int_{\Omega \backslash \Omega_{\ell}} v\left(\nabla u_{j}^{i k}(x)\right) g(x) \mathrm{d} x=\bar{V}\left(a_{i k}\right) \int_{\Omega \backslash \Omega_{\ell}} g(x) \mathrm{d} x .
$$

Particularly,

$$
\lim _{\ell \rightarrow \infty} \lim _{j \rightarrow \infty} \int_{\Omega \backslash \Omega_{\ell}} v\left(\nabla u_{j}^{i k}(x)\right) g(x) \mathrm{d} x=\bar{V}\left(a_{i k}\right) \lim _{\ell \rightarrow \infty} \int_{\Omega_{\backslash} \Omega_{\ell}} g(x) \mathrm{d} x=0 .
$$

By Lemma 4.3

$$
\bar{\Omega}=\bigcup_{i}\left\{x \in a_{i k}+\epsilon_{i k} \bar{\Omega}\right\} \bigcup N_{k},\left|N_{k}\right|=0 .
$$

We define a sequence of smooth cut-off functions $\left\{\eta_{\ell}\right\}_{\ell \in \mathbb{N}}$ such that

$$
\eta_{\ell}(x)=\left\{\begin{array}{cc}
0 & \text { in } \Omega_{\ell} \\
1 & \text { on } \partial \Omega
\end{array}\right.
$$

and $\left|\nabla \eta_{\ell}\right| \leq C \ell$ for some $C>0$.

Further, take a sequence $\left\{u_{k}^{\ell}\right\}_{k, \ell \in \mathbb{N}} \subset W^{1, p}\left(\Omega ; \mathbb{R}^{m}\right)$ defined by

$$
u_{k}^{\ell}(x)=\left\{\begin{array}{lc}
{\left[u\left(a_{i k}\right)+\epsilon_{i k} u_{j}^{i k}\left(\frac{x-a_{i k}}{\epsilon_{i k}}\right)\right]\left(1-\eta_{\ell}\left(\frac{x-a_{i k}}{\epsilon_{i k}}\right)\right)} & \\
+u(x) \eta_{\ell}\left(\frac{x-a_{i k}}{\epsilon_{i k}}\right) & \text { if } x \in a_{i k}+\epsilon_{i k} \Omega, \\
u(x) & \text { otherwise }
\end{array}\right.
$$

where $j=j(i, k, \ell)$ will be chosen later. Note that for every $k$ and $l$ we have $u_{k}^{l}-u \in W_{0}^{1, p}\left(\Omega, \mathbb{R}^{m}\right)$. 
We calculate for $x \in a_{i k}+\epsilon_{i k} \Omega$

$$
\begin{aligned}
\nabla u_{k}^{\ell}(x)= & \nabla u_{j}^{i k}\left(\frac{x-a_{i k}}{\epsilon_{i k}}\right)\left(1-\eta_{\ell}\left(\frac{x-a_{i k}}{\epsilon_{i k}}\right)\right) \\
& +\nabla u(x) \eta_{\ell}\left(\frac{x-a_{i k}}{\epsilon_{i k}}\right) \\
& +\frac{1}{\epsilon_{i k}}\left[u(x)-u\left(a_{i k}\right)-\epsilon_{i k} \nabla u\left(a_{i k}\right)\left(\frac{x-a_{i k}}{\epsilon_{i k}}\right)\right] \otimes \nabla \eta_{\ell}\left(\frac{x-a_{i k}}{\epsilon_{i k}}\right) \\
& +\left[\nabla u\left(a_{i k}\right)\left(\frac{x-a_{i k}}{\epsilon_{i k}}\right)-u_{j}^{i k}\left(\frac{x-a_{i k}}{\epsilon_{i k}}\right)\right] \otimes \nabla \eta_{\ell}\left(\frac{x-a_{i k}}{\epsilon_{i k}}\right) \\
= & A_{i k}^{\ell}(x)+B_{i k}^{\ell}(x)+C_{i k}^{\ell}(x)+D_{i k}^{\ell}(x)
\end{aligned}
$$

and let $A_{k}^{\ell}(x), B_{k}^{\ell}(x), C_{k}^{\ell}(x), D_{k}^{\ell}(x)$ be defined on the whole set $\Omega$ (up to a set of measure 0 ) by $A_{i k}^{\ell}(x), B_{i k}^{\ell}(x)$, $C_{i k}^{\ell}(x), D_{i k}^{\ell}(x)$ respectively on each set $a_{i k}+\epsilon_{i k} \Omega$.

Obviously, $\quad\left\{\left|B_{k}^{\ell}\right|^{p}\right\}_{k \in \mathbb{N}}$ is weakly compact in $L^{1}\left(\Omega ; \mathbb{R}^{m \times n}\right)$. Further, (4.18) implies that $\lim _{\ell \rightarrow \infty} \lim _{k \rightarrow \infty}\left\|C_{k}^{\ell}\right\|_{L^{p}\left(\Omega ; \mathbb{R}^{m \times n}\right)}^{p}=0$. Moreover, $\lim _{\ell \rightarrow \infty} \lim _{k \rightarrow \infty}\left\|D_{k}^{\ell}\right\|_{L^{p}\left(\Omega ; \mathbb{R}^{m \times n}\right)}^{p}=0$ if we take $j=j(i, k, l)$ so that $\int_{\Omega}\left|\nabla u\left(a_{i k}\right) x-u_{j}^{i k}(x)\right|^{p} \mathrm{~d} x<\frac{1}{l^{2 p}}$ due to (4.22).

Let us fix $k, i, \ell$. We can eventually enlarge each $j=j(i, k, \ell)$ so that additionally for any $\left(g, v_{0}\right) \in E_{k}$

$$
\left|\epsilon_{i k}^{n} \int_{\Omega} g\left(a_{i k}+\epsilon_{i k} y\right) v\left(\nabla u_{j}^{i k}(y)\right) \mathrm{d} y-\bar{V}\left(a_{i k}\right) \int_{a_{i k}+\epsilon_{i k} \Omega} g(x) \mathrm{d} x\right| \leq \frac{1}{2^{i} k}
$$

and

$$
\left|\epsilon_{i k}^{n} \int_{\Omega \backslash \Omega_{\ell}} g\left(a_{i k}+\epsilon_{i k} y\right) v\left(\nabla u_{j}^{i k}(y)\right) \mathrm{d} y-\epsilon_{i k}^{n} \bar{V}\left(a_{i k}\right) \int_{\Omega \backslash \Omega_{\ell}} g\left(a_{i k}+\epsilon_{i k} y\right) \mathrm{d} y\right| \leq \frac{1}{2^{i} k} .
$$

We have

$$
\begin{aligned}
& \int_{\Omega} g(x) v\left(\nabla u_{k}^{\ell}(x)\right) \mathrm{d} x=\sum_{i} \epsilon_{i k}^{n} \int_{\Omega} g\left(a_{i k}+\epsilon_{i k} y\right) v\left(\nabla u_{j}^{i k}(y)\right) \mathrm{d} y-\sum_{i} \epsilon_{i k}^{n} \int_{\Omega \backslash \Omega_{\ell}} g\left(a_{i k}+\epsilon_{i k} y\right) v\left(\nabla u_{j}^{i k}(y)\right) \mathrm{d} y \\
+ & \sum_{i} \epsilon_{i k}^{n} \int_{\Omega \backslash \Omega_{\ell}} g\left(a_{i k}+\epsilon_{i k} y\right) v\left(\nabla u_{k}^{\ell}\left(a_{i k}+\epsilon_{i k} y\right)\right) \mathrm{d} y=T_{k \ell}^{1}-T_{k \ell}^{2}+T_{k \ell}^{3} .
\end{aligned}
$$

We see that

$$
\begin{aligned}
\lim _{\ell \rightarrow \infty} \lim _{k \rightarrow \infty} T_{k \ell}^{1} & =\lim _{k \rightarrow \infty} \sum_{i} \bar{V}\left(a_{i k}\right) \int_{a_{i k}+\epsilon_{i k} \Omega} g(x) \mathrm{d} x=\int_{\Omega} \bar{V}(x) g(x) \mathrm{d} x \\
& =\int_{\Omega} \int_{\beta_{\mathcal{R}} \mathbb{R}^{m \times n}} v_{0}(s) \hat{\nu}_{x}(\mathrm{~d} s) g(x) \sigma(\mathrm{d} x) .
\end{aligned}
$$

Applying (4.20) with $g=1$ yields

$$
\lim _{k \rightarrow \infty} \sum_{i}\left|\bar{V}\left(a_{i k}\right)\right| \epsilon_{i k}^{n}|\Omega|=\int_{\Omega}|\bar{V}(x)| \mathrm{d} x
$$


Therefore, we have

$$
\begin{aligned}
\lim _{\ell \rightarrow \infty} \lim _{k \rightarrow \infty}\left|T_{k \ell}^{2}\right| & =\lim _{\ell \rightarrow \infty} \lim _{k \rightarrow \infty}\left|\sum_{i} \epsilon_{i k}^{n} \bar{V}\left(a_{i k}\right) \int_{\Omega \backslash \Omega_{\ell}} g\left(a_{i k}+\epsilon_{i k} y\right) \mathrm{d} y\right| \\
& \leq \lim _{\ell \rightarrow \infty} \lim _{k \rightarrow \infty}\|g\|_{C(\bar{\Omega})} \frac{\left|\Omega \backslash \Omega_{\ell}\right|}{|\Omega|} \sum_{i} \epsilon_{i k}^{n}|\Omega|\left|\bar{V}\left(a_{i k}\right)\right| \\
& =\lim _{\ell \rightarrow \infty} \frac{\left|\Omega \backslash \Omega_{\ell}\right|}{|\Omega|}\|g\|_{C(\bar{\Omega})} \int_{\Omega}|\bar{V}(x)| \mathrm{d} x=0
\end{aligned}
$$

because $\left|\Omega \backslash \Omega_{\ell}\right| \rightarrow 0$. We show that also $\lim _{\ell \rightarrow \infty} \lim _{k \rightarrow \infty} T_{k \ell}^{3}=0$. Indeed, for a constant $\tilde{C}>0$ we have

$$
\begin{aligned}
& \left|\sum_{i} \epsilon_{i k}^{n} \int_{\Omega \backslash \Omega_{\ell}} g\left(a_{i k}+\epsilon_{i k} y\right) v\left(\nabla u_{k}^{\ell}\left(a_{i k}+\epsilon_{i k} y\right)\right) \mathrm{d} y\right| \leq \tilde{C} \sum_{i} \epsilon_{i k}^{n} \int_{\Omega \backslash \Omega_{\ell}}\left(1+\left|\nabla u_{j}^{i k}(y)\right|^{p}\right) \mathrm{d} y \\
+ & \tilde{C} \sum_{i} \epsilon_{i k}^{n} \int_{\Omega \backslash \Omega_{\ell}}\left|B_{k}^{\ell}\left(a_{i k}+\epsilon_{i k} y\right)\right|^{p} \mathrm{~d} y+\tilde{C} \sum_{i} \epsilon_{i k}^{n} \int_{\Omega \backslash \Omega_{\ell}}\left|C_{k}^{\ell}\left(a_{i k}+\epsilon_{i k} y\right)\right|^{p} \mathrm{~d} y \\
+ & \tilde{C} \sum_{i} \epsilon_{i k}^{n} \int_{\Omega \backslash \Omega_{\ell}}\left|D_{k}^{\ell}\left(a_{i k}+\epsilon_{i k} y\right)\right|^{p} \mathrm{~d} y=J_{k l}^{1}+J_{k l}^{2}+J_{k l}^{3}+J_{k l}^{4} .
\end{aligned}
$$

We prove that $P_{t}:=\lim _{l \rightarrow \infty} \lim _{k \rightarrow \infty} J_{k l}^{t}=0$ for every $t \in\{1,2,3,4\}$. Indeed,

$$
\begin{aligned}
P_{3} / \tilde{C} & =\lim _{\ell \rightarrow \infty} \lim _{k \rightarrow \infty} J_{k l}^{3} / \bar{C}=\lim _{\ell \rightarrow \infty} \lim _{k \rightarrow \infty} \sum_{i} \int_{a_{i k}+\epsilon_{i k}\left(\Omega \backslash \Omega_{\ell}\right)}\left|C_{k}^{\ell}(y)\right|^{p} \mathrm{~d} y \leq \lim _{\ell \rightarrow \infty} \lim _{k \rightarrow \infty} \sum_{i} \int_{a_{i k}+\epsilon_{i k} \Omega}\left|C_{k}^{\ell}(y)\right|^{p} \mathrm{~d} y \\
& =\lim _{\ell \rightarrow \infty} \lim _{k \rightarrow \infty} \int_{\Omega}\left|C_{k}^{\ell}(y)\right|^{p} \mathrm{~d} y=0
\end{aligned}
$$

and by almost the same arguments $P_{4}=0$. We also have $P_{1}=0$ due to (4.27) computed for $v_{0}=1$ and $g=1$ and $P_{2}=0$ because the sequence $\left\{\left|B_{k}^{\ell}\right|^{p}\right\}_{k \in \mathbb{N}}$ is weakly compact in $L^{1}(\Omega)$.

Consequently, for all $\left(g, v_{0}\right) \in \Gamma \times S$

$$
\lim _{\ell \rightarrow \infty} \lim _{k \rightarrow \infty} \int_{\Omega} g(x) v\left(\nabla u_{k}^{\ell}(x)\right) \mathrm{d} x=\int_{\Omega} \int_{\beta_{\mathcal{R}} \mathbb{R}^{m \times n}} v_{0}(s) \hat{\nu}_{x}(\mathrm{~d} s) g(x) \sigma(\mathrm{d} x) .
$$

The proof is finished now by Lemma 3.3. The fact that $\left\{u_{k}\right\}$ can be chosen to have the same boundary conditions as $u$ follows from construction of $u_{k}^{l}$.

Remark 4.5. No regularity of the domain $\Omega$ other than $|\partial \Omega|=0$ is required for this proof. The only place where it could play a role is (4.17). But it is true for every $\Omega$ because $w_{a, \epsilon}(y)$ uses the values of $u$ only in the set $a+\epsilon \Omega$ which is contained in $\Omega$ together with its certain neighborhood.

Finally, we prove the general result with $\sigma$ having possibly also a singular part.

Proposition 4.6. Let $\Omega$ be an arbitrary bounded domain such that $|\partial \Omega|=0,1<p<+\infty$ and $(\sigma, \hat{\nu}) \in$ $\mathcal{D} \mathcal{M}_{\mathcal{R}}^{p}\left(\Omega ; \mathbb{R}^{m \times n}\right)$ be such that the following three conditions hold:

$$
\exists u \in W^{1, p}\left(\Omega ; \mathbb{R}^{m}\right): \text { for a.a.x } \in \Omega \nabla u(x)=d_{\sigma}(x) \int_{\mathbb{R}^{m \times n}} \frac{s}{1+|s|^{p}} \hat{\nu}_{x}(\mathrm{~d} s),
$$


for almost all $x \in \Omega$ and for any $v \in \Upsilon_{\mathcal{R}}^{p}\left(\mathbb{R}^{m \times n}\right)$ the following inequality is fulfilled

$$
Q v(\nabla u(x)) \leq d_{\sigma}(x) \int_{\beta_{\mathcal{R}} \mathbb{R}^{m \times n}} \frac{v(s)}{1+|s|^{p}} \hat{\nu}_{x}(\mathrm{~d} s),
$$

for $\sigma$-almost all $x \in \bar{\Omega}$ and all $v \in \Upsilon_{\mathcal{R}}^{p}\left(\mathbb{R}^{m \times n}\right)$ with $Q v>-\infty$ it holds that

$$
0 \leq \int_{\beta_{\mathcal{R} \mathbb{R}^{m \times n} \backslash \mathbb{R}^{m \times n}}} \frac{v(s)}{1+|s|^{p}} \hat{\nu}_{x}(\mathrm{~d} s) .
$$

Then $(\sigma, \hat{\nu}) \in \mathcal{G D} \mathcal{M}_{\mathcal{R}}^{p}\left(\Omega ; \mathbb{R}^{m \times n}\right)$. Moreover, its generating sequence, $\left\{\nabla u_{k}\right\}_{k \in \mathbb{N}}$, can be chosen in the way that $\left\{u_{k}-u\right\}_{k \in \mathbb{N}} \subset W_{0}^{1, p}\left(\Omega, \mathbb{R}^{m}\right)$.

Proof. Notice that if the singular part of $\sigma$ vanishes then the assertion follows from Proposition 4.4. Hence, we suppose that $\sigma_{s} \neq 0$. The proof is divided into two steps.

(i) We first suppose that the singular part of $\sigma, \sigma_{s}$, consists of a finite sum of atoms, i.e., $\sigma_{s}=\sum_{i=1}^{N} a_{i} \delta_{x_{i}}$, where $a_{i}>0$ and $x_{i} \in \Omega, 1 \leq i \leq N$.

First, note that by Lemma 2.3 inevitably $\int_{\beta_{\mathcal{R}} \mathbb{R}^{m \times n} \backslash \mathbb{R}^{m \times n}} \hat{\nu}_{x_{i}}(\mathrm{~d} s)=1$ for $1 \leq i \leq N$. We define $B\left(x_{i}, r\right) \subset \Omega$ such that $B\left(x_{i}, r\right)=\left\{x \in \Omega ;\left|x_{i}-x\right|<r\right\}$ for $r>0$ sufficiently small $, i=1, \ldots, N$, and $B\left(x_{i}, r\right) \cap B\left(x_{j}, r\right)=\emptyset$ if $i \neq j$. We define for $i=1, \ldots, N$

$$
\lambda_{i}(r)=\frac{1}{a_{i}} \int_{B\left(x_{i}, r\right)}\left(1+|\nabla u(x)|^{p}\right) \mathrm{d} x .
$$

As $\lim _{r \rightarrow 0} \lambda_{i}(r)=0$ we will only consider $r<r_{0}$ for $r_{0}>0$ so small that $0<\lambda_{i}(r)<1$.

Further, put for a.a. $x \in \Omega$

$$
\hat{\nu}_{x}^{r}= \begin{cases}\hat{\nu}_{x} & \text { if } x \in \bar{\Omega} \backslash \cup_{i=1}^{N} B\left(x_{i}, r\right) \\ \lambda_{i}(r) \delta_{\nabla u(x)}+\left(1-\lambda_{i}(r)\right) \hat{\nu}_{x_{i}} & \text { if } x \in B\left(x_{i}, r\right)\end{cases}
$$

and the measure $\sigma_{r}=d_{\sigma_{r}} \mathrm{~d} x$ defined through its density $d_{\sigma_{r}}$ as

$$
d_{\sigma_{r}}(x)= \begin{cases}d_{\sigma}(x) & \text { if } x \in \bar{\Omega} \backslash \cup_{i=1}^{N} B\left(x_{i}, r\right) \\ \frac{1+|\nabla u(x)|^{p}}{\lambda_{i}(r)} & \text { if } x \in B\left(x_{i}, r\right) .\end{cases}
$$

It is easy to verify by means of Proposition 4.4 that $\left(\sigma_{r}, \hat{\nu}^{r}\right) \in \mathcal{D} \mathcal{M}_{\mathcal{R}}^{p}\left(\Omega ; \mathbb{R}^{m \times n}\right)$. We see that for almost all $x \in \Omega$

$$
d_{\sigma_{r}}(x) \int_{\mathbb{R}^{m \times n}} \frac{s}{1+|s|^{p}} \hat{\nu}_{x}^{r}(\mathrm{~d} s)=\nabla u(x)
$$

and that due to (4.30) for almost all $x \in B\left(x_{i}, r\right)$

$$
\frac{\lambda_{i}(r)(Q v(\nabla u(x))-v(\nabla u(x)))}{\left(1-\lambda_{i}(r)\right)\left(1+|\nabla u(x)|^{p}\right)} \leq 0 \leq \int_{\beta_{\mathcal{R}} \mathbb{R}^{m \times n} \backslash \mathbb{R}^{m \times n}} \frac{v(s)}{1+|s|^{p}} \hat{\nu}_{x_{i}}(\mathrm{~d} s) .
$$

Altogether we have for any $v \in \Upsilon_{\mathcal{R}}^{p}\left(\mathbb{R}^{m \times n}\right)$ with $Q v>-\infty$

$$
Q v(\nabla u(x)) \leq d_{\sigma_{r}}(x) \int_{\beta_{\mathcal{R} \mathbb{R}^{m \times n}}} \frac{v(s)}{1+|s|^{p}} \hat{\nu}_{x}^{r}(\mathrm{~d} s)
$$


and by Proposition 4.4 there is $\left\{u_{k}^{r}\right\} \in W^{1, p}\left(\Omega ; \mathbb{R}^{m}\right)$ such that $\left\{\nabla u_{k}^{r}\right\}_{k \in \mathbb{N}}$ generates $\left(\sigma_{r}, \hat{\nu}^{r}\right) \in \mathcal{G} \mathcal{D} \mathcal{M}_{\mathcal{R}}^{p}\left(\Omega ; \mathbb{R}^{m \times n}\right)$ and $\left\{u_{k}-u\right\}_{k \in \mathbb{N}} \subset W_{0}^{1, p}\left(\Omega, \mathbb{R}^{m \times n}\right)$.

We calculate for any $v_{0} \in \mathcal{R}$ and $g \in C(\bar{\Omega})$

$$
\begin{aligned}
& \lim _{r \rightarrow 0} \int_{\bar{\Omega}} \int_{\beta_{\mathcal{R}} \mathbb{R}^{m \times n}} v_{0}(s) \hat{\nu}_{x}^{r}(\mathrm{~d} s) g(x) \sigma_{r}(\mathrm{~d} x)=\lim _{r \rightarrow 0} \int_{\bar{\Omega} \backslash \cup_{i=1}^{N} B\left(x_{i}, r\right)} \int_{\beta_{\mathcal{R}} \mathbb{R}^{m \times n}} v_{0}(s) \hat{\nu}_{x}(\mathrm{~d} s) g(x) d_{\sigma}(x) \mathrm{d} x \\
+ & \lim _{r \rightarrow 0} \sum_{i=1}^{N} \int_{B\left(x_{i}, r\right)} v(\nabla u(x)) g(x) \mathrm{d} x \\
+ & \lim _{r \rightarrow 0} \sum_{i=1}^{N} \frac{1-\lambda_{i}(r)}{\lambda_{i}(r)} \int_{B\left(x_{i}, r\right)} g(x)\left(1+|\nabla u(x)|^{p}\right) \mathrm{d} x \int_{\beta_{\mathcal{R}} \mathbb{R}^{m \times n}} v_{0}(s) \hat{\nu}_{x_{i}}(\mathrm{~d} s)=: I+I I+I I I .
\end{aligned}
$$

Obviously, $I+I I=\int_{\bar{\Omega}} \int_{\beta_{\mathcal{R}} \mathbb{R}^{m \times n}} v_{0}(s) \hat{\nu}_{x}(\mathrm{~d} s) g(x) d_{\sigma}(x) \mathrm{d} x$, while

$$
\begin{aligned}
& I I I=\lim _{r \rightarrow 0} \sum_{i=1}^{N} \frac{1}{\lambda_{i}(r)} \int_{B\left(x_{i}, r\right)} g(x)\left(1+|\nabla u(x)|^{p}\right) \mathrm{d} x \int_{\beta_{\mathcal{R}} \mathbb{R}^{m \times n}} v_{0}(s) \hat{\nu}_{x_{i}}(\mathrm{~d} s) \\
& =\sum_{i=1}^{N} a_{i}\left(\int_{\beta_{\mathcal{R}} \mathbb{R}^{m \times n}} v_{0}(s) \hat{\nu}_{x_{i}}(\mathrm{~d} s)\right) \lim _{r \rightarrow 0} \frac{1}{\int_{B\left(x_{i}, r\right)}\left(1+|\nabla u(x)|^{p}\right) \mathrm{d} x} \int_{B\left(x_{i}, r\right)} g(x)\left(1+|\nabla u(x)|^{p}\right) \mathrm{d} x \\
& =\sum_{i=1}^{N} a_{i} g\left(x_{i}\right) \int_{\beta_{\mathcal{R}} \mathbb{R}^{m \times n}} v_{0}(s) \hat{\nu}_{x_{i}}(\mathrm{~d} s)=\int_{\bar{\Omega}} \int_{\beta_{\mathcal{R}} \mathbb{R}^{m \times n}} v_{0}(s) \hat{\nu}_{x}(\mathrm{~d} s) g(x) \sigma_{s}(\mathrm{~d} x) .
\end{aligned}
$$

Finally, it yields

$$
\lim _{r \rightarrow 0} \lim _{k \rightarrow \infty} \int_{\Omega} v\left(\nabla u_{k}^{r}(x)\right) g(x) \mathrm{d} x=\int_{\bar{\Omega}} \int_{\beta_{\mathcal{R}} \mathbb{R}^{m \times n}} v_{0}(s) \hat{\nu}_{x}(\mathrm{~d} s) g(x) \sigma(\mathrm{d} x) .
$$

Lemma 3.3 implies the existence of a bounded sequence $\left\{\nabla u_{k}\right\}_{k \in \mathbb{N}}$ such that $\left\{u_{k}-u\right\}_{k \in \mathbb{N}} \subset W_{0}^{1, p}\left(\Omega, \mathbb{R}^{m \times n}\right)$ and

$$
\lim _{k \rightarrow \infty} \int_{\Omega} v\left(\nabla u_{k}(x)\right) g(x) \mathrm{d} x=\int_{\bar{\Omega}} \int_{\beta_{\mathcal{R}} \mathbb{R}^{m \times n}} v_{0}(s) \hat{\nu}_{x}(\mathrm{~d} s) g(x) \sigma(\mathrm{d} x)
$$

whenever $v \in \Upsilon_{\mathcal{R}}^{p}\left(\mathbb{R}^{m \times n}\right)$ and $g \in C(\bar{\Omega})$.

(ii) Now we prove a general case. Take $l \in \mathbb{N}$. There exists a finite partition $\mathcal{P}_{l}=\left\{\Omega_{j}^{l}\right\}_{j=1}^{J(l)}$ of $\bar{\Omega}$ such that $\Omega_{j_{1}}^{l} \cap \Omega_{j_{2}}=\emptyset, 1 \leq j_{1}<j_{2} \leq J(l)$ and all $\Omega_{j}^{l}$ are measurable with $\operatorname{diam}\left(\Omega_{j}^{l}\right)<1 / l$. Besides, we may suppose that, for any $l \in \mathbb{N}$, the partition $\mathcal{P}_{l+1}$ is a refinement of $\mathcal{P}_{l}$ and that $\operatorname{int}\left(\Omega_{j}^{l}\right) \neq \emptyset$ for all $j$. Let $\sigma_{s}$ be the singular part of $\sigma$. We set $a_{i}^{l}=\sigma_{s}\left(\Omega_{i}^{l}\right)$, where $\sigma_{s}$ is the singular part of $\sigma$. Let us put

$$
N(l)=\left\{1 \leq j \leq J(l) ; a_{j}^{l} \neq 0\right\}
$$

take if $i \in N(l)$ take $x_{i} \in \operatorname{int}\left(\Omega_{i}^{l}\right)$ and define a measure $\left(\sigma^{l}, \hat{\nu}^{l}\right)$ by the formula $\sigma^{l}(\mathrm{~d} x)=d_{\sigma}(x)+\sum_{i \in N(l)} a_{i}^{l} \delta_{x_{i}}$ and

$$
\hat{\nu}_{x}^{l}= \begin{cases}\hat{\nu}_{x} & \text { if } x \neq x_{i} \\ \hat{\nu}_{x_{i}}^{l} & \text { if } x=x_{i}\end{cases}
$$


where supp $\hat{\nu}_{x_{i}}^{l} \subset \beta_{\mathcal{R}} \mathbb{R}^{m \times n} \backslash \mathbb{R}^{m \times n}$ and for any $v_{0} \in \mathcal{R}$

$$
\int_{\beta_{\mathcal{R}} \mathbb{R}^{m \times n}} v_{0}(s) \hat{\nu}_{x_{i}}^{l}(\mathrm{~d} s)=\frac{1}{\sigma_{s}\left(\Omega_{i}^{l}\right)} \int_{\Omega_{i}^{l}} \int_{\beta_{\mathcal{R}} \mathbb{R}^{m \times n}} v_{0}(s) \hat{\nu}_{x}(\mathrm{~d} s) \sigma_{s}(\mathrm{~d} x) .
$$

Using Lemma 2.6 we can equivalently rewrite (4.36) as

$$
\int_{\beta_{\mathcal{R}} \mathbb{R}^{m \times n} \backslash \mathbb{R}^{m \times n}} v_{0}(s) \hat{\nu}_{x_{i}}^{l}(\mathrm{~d} s)=\frac{1}{\sigma_{s}\left(\Omega_{i}^{l}\right)} \int_{\Omega_{i}^{l}} \int_{\beta_{\mathcal{R}} \mathbb{R}^{m \times n} \backslash \mathbb{R}^{m \times n}} v_{0}(s) \hat{\nu}_{x}(\mathrm{~d} s) \sigma_{s}(\mathrm{~d} x) .
$$

Part (i) implies $\left(\sigma^{l}, \hat{\nu}^{l}\right) \in \mathcal{G} \mathcal{D} \mathcal{M}_{\mathcal{R}}^{p}\left(\Omega ; \mathbb{R}^{m \times n}\right)$. Indeed, the fact that $\left(\sigma^{l}, \hat{\nu}^{l}\right) \in \mathcal{D} \mathcal{M}_{\mathcal{R}}^{p}\left(\Omega ; \mathbb{R}^{m \times n}\right)$ is checked by using Proposition 2.4. Moreover, an easy verification shows that (4.28),(4.29) and (4.30) are also satisfied for $\left(\sigma^{l}, \hat{\nu}^{l}\right)$ and $(4.28)$ holds with the same function $u$.

Let $\left\{u_{k}^{l}\right\}_{k \in \mathbb{N}} \subset W^{1, p}\left(\Omega ; \mathbb{R}^{m}\right)$ be such that $\left\{\nabla u_{k}^{l}\right\}_{k \in \mathbb{N}}$ generates $\left(\sigma^{l}, \hat{\nu}^{l}\right)$ and additionally $\left\{u_{k}^{l}-u\right\}_{k} \subset$ $W_{0}^{1, p}\left(\Omega, \mathbb{R}^{m}\right)$. We have for any $l \in \mathbb{N}$

$$
\lim _{k \rightarrow \infty} \int_{\Omega}\left(1+\left|\nabla u_{k}^{l}(x)\right|^{p}\right) \mathrm{d} x=\sigma^{l}(\bar{\Omega})=\sigma(\bar{\Omega})
$$

and for any $v_{0} \in \mathcal{R}$ and any $g \in C(\bar{\Omega})$

$$
\begin{aligned}
& \lim _{l \rightarrow \infty}\left|\int_{\bar{\Omega}} \int_{\beta_{\mathcal{R}} \mathbb{R}^{m \times n}} v_{0}(s) \hat{\nu}_{x}^{l}(\mathrm{~d} s) g(x) \sigma^{l}(\mathrm{~d} x)-\int_{\bar{\Omega}} \int_{\beta_{\mathcal{R}} \mathbb{R}^{m \times n}} v_{0}(s) \hat{\nu}_{x}(\mathrm{~d} s) g(x) \sigma(\mathrm{d} x)\right| \\
& =\lim _{l \rightarrow \infty}\left|\sum_{i \in N(l)} g\left(x_{i}\right) \sigma_{s}\left(\Omega_{i}^{l}\right) \int_{\beta_{\mathcal{R}} \mathbb{R}^{m \times n} \backslash \mathbb{R}^{m \times n}} v_{0}(s) \hat{\nu}_{x_{i}}^{l}(\mathrm{~d} s)-\int_{\bar{\Omega}} \int_{\beta_{\mathcal{R}} \mathbb{R}^{m \times n} \backslash \mathbb{R}^{m \times n}} v_{0}(s) \hat{\nu}_{x}(\mathrm{~d} s) g(x) \sigma_{s}(\mathrm{~d} x)\right| \\
& =\lim _{l \rightarrow \infty}\left|\sum_{i \in N(l)}\left(\int_{\Omega_{i}^{l}} \int_{\beta_{\mathcal{R}} \mathbb{R}^{m \times n} \backslash \mathbb{R}^{m \times n}} v_{0}(s) \hat{\nu}_{x}(\mathrm{~d} s) g\left(x_{i}\right) \sigma_{s}(\mathrm{~d} x)-\int_{\Omega_{i}^{l}} \int_{\beta_{\mathcal{R} \mathbb{R}^{m \times n} \backslash \mathbb{R}^{m \times n}}} v_{0}(s) \hat{\nu}_{x}(\mathrm{~d} s) g(x) \sigma_{s}(\mathrm{~d} x)\right)\right| \\
& \leq \lim _{l \rightarrow \infty} \sum_{i \in N(l)} \int_{\Omega_{i}^{l}} \int_{\beta_{\mathcal{R}} \mathbb{R}^{m \times n} \backslash \mathbb{R}^{m \times n}}\left|v_{0}(s)\right| \hat{\nu}_{x}(\mathrm{~d} s)\left|g(x)-g\left(x_{i}\right)\right| \sigma_{s}(\mathrm{~d} x) \leq C \sigma_{s}(\bar{\Omega}) \lim _{l \rightarrow \infty} M_{g}\left(\frac{1}{l}\right)=0,
\end{aligned}
$$

where $\left|v_{0}\right| \leq C$. Hence, we get for any $v \in \Upsilon_{\mathcal{R}}^{p}\left(\mathbb{R}^{m \times n}\right)$ and any $g \in C(\bar{\Omega})$

$$
\lim _{l \rightarrow \infty} \lim _{k \rightarrow \infty} \int_{\Omega} v\left(\nabla u_{k}^{l}(x)\right) g(x) \mathrm{d} x=\int_{\bar{\Omega}} \int_{\beta_{\mathcal{R}} \mathbb{R}^{m \times n}} v_{0}(s) \hat{\nu}_{x}(\mathrm{~d} s) g(x) \sigma(\mathrm{d} x)
$$

and we finish the proof by using Lemma 3.3.

Proof of Theorem 2.7. It follows directly from Propositions 3.8 and 4.6.

Remark 4.7. Theorem 2.8 is a part of Proposition 3.8 . 


\section{Proofs of lower Semicontinuity Theorems 2.9 And 2.10}

Proof of Theorem 2.9. Let $\mathcal{R}$ be an arbitrary separable complete closed ring containing $v /\left(1+|\cdot|^{p}\right)$ and corresponding to the compactification $\beta_{\mathcal{R}} \mathbb{R}^{m \times n}$ of $\mathbb{R}^{m \times n}$. After extracting the subsequence we may suppose that $\left\{\nabla u_{k}\right\}_{k \in \mathbb{N}}$ generates a DiPerna-Majda measure $(\sigma, \hat{\nu}) \in \mathcal{G} \mathcal{D} \mathcal{M}_{\mathcal{R}}^{p}\left(\Omega ; \mathbb{R}^{m \times n}\right)$ and we have $($ see $(2.12))$

$$
\begin{aligned}
\lim _{k \rightarrow \infty} I\left(u_{k}\right)= & \int_{\Omega} \int_{\mathbb{R}^{m \times n}} v(s) \nu_{x}(\mathrm{~d} s) g(x) \mathrm{d} x \\
& +\int_{\bar{\Omega}} \int_{\beta_{\mathcal{R}} \mathbb{R}^{m \times n} \backslash \mathbb{R}^{m \times n}} \frac{v(s)}{1+|s|^{p}} \hat{\nu}_{x}(\mathrm{~d} s) g(x) \sigma(\mathrm{d} x),
\end{aligned}
$$

where $\nu \in \mathcal{G Y}^{p}\left(\Omega ; \mathbb{R}^{m \times n}\right)$ and $(\sigma, \hat{\nu}) \in \mathcal{D} \mathcal{M}_{\mathcal{R}}^{p}\left(\Omega ; \mathbb{R}^{m \times n}\right)$ are gradient Young and DiPerna-Majda measures generated by $\left\{\nabla_{k}\right\}_{k \in \mathbb{N}}$, respectively.

Now, the sequential weak lower semicontinuity of $I$ follows from Theorem 2.8 and (2.24). Indeed, if (i) or (iii) holds Theorem 2.8 shows that

$$
\int_{\bar{\Omega}} \int_{\beta_{\mathcal{R}} \mathbb{R}^{m \times n} \backslash \mathbb{R}^{m \times n}} \frac{v(s)}{1+|s|^{p}} \hat{\nu}_{x}(\mathrm{~d} s) g(x) \sigma(\mathrm{d} x) \geq 0 .
$$

If (ii) is valid, we decompose the left-hand side of (5.2) to

$$
\int_{\bar{\Omega}} \int_{\beta_{\mathcal{R}} \mathbb{R}^{m \times n} \backslash \mathbb{R}^{m \times n}} \frac{v^{+}(s)}{1+|s|^{p}} \hat{\nu}_{x}(\mathrm{~d} s) g(x) \sigma(\mathrm{d} x)-\int_{\bar{\Omega}} \int_{\beta_{\mathcal{R}} \mathbb{R}^{m \times n} \backslash \mathbb{R}^{m \times n}} \frac{v^{-}(s)}{1+|s|^{p}} \hat{\nu}_{x}(\mathrm{~d} s) g(x) \sigma(\mathrm{d} x)
$$

and realize that $v^{+}:=\max (v, 0) \geq 0$ and due to (ii)

$$
\int_{\bar{\Omega}} \int_{\beta_{\mathcal{R} \mathbb{R}^{m \times n} \backslash \mathbb{R}^{m \times n}}} \frac{v^{-}(s)}{1+|s|^{p}} \hat{\nu}_{x}(\mathrm{~d} s) g(x) \sigma(\mathrm{d} x)=0,
$$

i.e., (5.2) holds again.

Proof of Theorem 2.10. Let us first prove the "only if part". Hence, suppose that $I$ is sequentially weakly lower semicontinuous. Taking $\left\{w_{k}\right\}$ as in the theorem we have for any weakly convergent subsequence (not relabeled) that $w_{k} \rightarrow c$ in $W^{1, p}\left(\Omega ; \mathbb{R}^{m}\right)$, where $c$ is a constant. Indeed, $\nabla w_{k}$ converges in measure which means that it generates the Young measure $\nu_{x}=\delta_{0}$ for a.a. $x \in \Omega$ and, particularly, $\nabla w_{k} \rightarrow 0$ in $L^{p}\left(\Omega ; \mathbb{R}^{m \times n}\right)$. By sequential weak lower semicontinuity of $I$ we have $\liminf _{k \rightarrow \infty} I\left(w_{k}\right) \geq I(c)=I(0)$.

Now we are going to prove the "if part". Let us take any bounded $\left\{u_{k}\right\} \subset W^{1, p}\left(\Omega ; \mathbb{R}^{m}\right)$ such that w$\lim _{k \rightarrow \infty} u_{k}=u$. Suppose that a subsequence of $\left\{\nabla u_{k}\right\}$ (not relabeled) generates $(\sigma, \hat{\nu}) \in \mathcal{G D} \mathcal{M}_{\mathcal{R}}^{p}\left(\Omega ; \mathbb{R}^{m \times n}\right)$. Using Lemma 2.2 and its notation we decompose $u_{k}=z_{k}+w_{k}$ for any $k \in \mathbb{N}$. Then (3.13) and the assumption $\liminf _{k \rightarrow \infty} I\left(w_{k}\right) \geq I(0)$ imply that

$$
\int_{\bar{\Omega}} \int_{\beta_{\mathcal{R}} \mathbb{R}^{m \times n} \backslash \mathbb{R}^{m \times n}} \frac{v(s)}{1+|s|^{p}} \hat{\nu}_{x}(\mathrm{~d} s) g(x) \mathrm{d} x \geq 0
$$

for any subsequence of $\left\{w_{k}\right\}$ (not relabeled) such that $I\left(w_{k}\right)$ converges. Let $\left\{\nabla u_{k}\right\}_{k \in \mathbb{N}}$ generate a gradient Young measure $\nu=\left\{\nu_{x}\right\}_{x \in \Omega} \in \mathcal{G} \mathcal{Y}^{p}\left(\Omega ; \mathbb{R}^{m \times n}\right)$. We have using (2.12)

$$
\begin{aligned}
\lim _{k \rightarrow \infty} \int_{\Omega} g(x) v\left(\nabla u_{k}(x)\right) \mathrm{d} x= & \int_{\Omega} \int_{\mathbb{R}^{m \times n}} v(s) \nu_{x}(\mathrm{~d} s) g(x) \mathrm{d} x \\
& +\int_{\bar{\Omega}} \int_{\beta_{\mathcal{R}} \mathbb{R}^{m \times n} \backslash \mathbb{R}^{m \times n}} \frac{v(s)}{1+|s|^{p}} \hat{\nu}_{x}(\mathrm{~d} s) g(x) \sigma(\mathrm{d} x) \geq \int_{\Omega} v(\nabla u(x)) g(x) \mathrm{d} x .
\end{aligned}
$$


The last inequality follows from (5.4) and from Kinderlehrer's and Pedregal's characterization of gradient Young measures (2.24). The theorem is proved.

Acknowledgements. This work was initiated during M.K's visit of the Institute of Mathematics of the Warsaw University and the visit of both authors in the Institute of Mathematics of the Polish Academy of Sciences and continued during the visit of A.K. in the Inst. of Inf. Theory and Automation of the Academy of Science of the Czech Republic and of Charles University, Prague. Moreover, M.K. thanks the IMA at the University of Minnesota and Caesar, Bonn for the support. The hospitality of all involved institutions is gratefully acknowledged. We thank M. Hušek, J. Kristensen, and T. Roubíček for fruitful discussions.

\section{REFERENCES}

[1] E. Acerbi and N. Fusco, Semicontinuity problems in the calculus of variations. Arch. Rational Mech. Anal. 86 (1984) $125-145$.

[2] J.J. Alibert and G. Bouchitté, Non-uniform integrability and generalized Young measures. J. Convex Anal. 4 (1997) 125-145.

[3] W. Allard, On the first variation of a varifold. Ann. Math. 95 (1972) 417-491.

[4] F.J. Almgren Jr., Existence and regularity almost everywhere of solutions to elliptic variational problems among surfaces of varying topological type and singularity structure. Ann. Math. 87 (1968) 321-391.

[5] J.M. Ball, A version of the fundamental theorem for Young measures, in PDEs and Continuum Models of Phase Transition, M. Rascle, D. Serre and M. Slemrod Eds., Lect. Notes Phys. 344, Springer, Berlin (1989) 207-215.

[6] J.M Ball and F. Murat, $W^{1, p}$-quasiconvexity and variational problems for multiple integrals. J. Funct. Anal. 58 (1984) $225-253$.

[7] P. Billingsley, Probability and Measure. 3rd edn., John Wiley \& Sons Ltd., Chichester (1995).

[8] B. Dacorogna, Direct Methods in the Calculus of Variations. Springer, Berlin (1989).

[9] R.J. DiPerna and A.J. Majda, Oscillations and concentrations in weak solutions of the incompressible fluid equations. Commun. Math. Phys. 108 (1987) 667-689.

[10] N. Dunford and J.T. Schwartz, Linear Operators, Part I. Interscience, New York (1967).

[11] R. Engelking, General topology. 2nd edn., PWN, Warszawa (1985).

[12] L.C. Evans and R.F. Gariepy, Measure Theory and Fine Properties of Functions. CRC Press, Inc. Boca Raton (1992).

[13] I. Fonseca, Lower semicontinuity of surface energies. Proc. Roy. Soc. Edinburgh 120A (1992) 95-115.

[14] I. Fonseca and S. Müller, P. Pedregal, Analysis of concentration and oscillation effects generated by gradients. SIAM J. Math. Anal. 29 (1998) 736-756.

[15] L. Greco, T. Iwaniec and U. Subramanian, Another approach to biting convergence of Jacobians. Illin. Journ. Math. 47 (2003) 815-830.

[16] M. de Guzmán, Differentiation of integrals in $\mathbb{R}^{n}$, Lecture Notes in Math. 481. Springer, Berlin (1975).

[17] O.M Hafsa, J.-P. Mandallena and G. Michaille, Homogenization of periodic nonconvex integral functionals in terms of Young measures. ESAIM: COCV 12 (2006) 35-51.

[18] A. Kałamajska, On lower semicontinuity of multiple integrals. Coll. Math. 74 (1997) 71-78.

[19] A. Kałamajska, On Young measures controlling discontinuous functions. J. Conv. Anal. 13 (2006) 177-192.

[20] A. Kałamajska and M. Kružík, On weak lower semicontinuity of integral functionals along concentrating sequences (in preparation).

[21] D. Kinderlehrer and P. Pedregal, Characterization of Young measures generated by gradients. Arch. Rat. Mech.Anal. 115 (1991) 329-365.

[22] D. Kinderlehrer and P. Pedregal, Gradient Young measures generated by sequences in Sobolev spaces. J. Geom. Anal. 4 (1994) 59-90.

[23] J. Kristensen, Finite functionals and Young measures generated by gradients of Sobolev functions. Mat-report 1994-34, Math. Institute, Technical University of Denmark (1994).

[24] J. Kristensen, Lower semicontinuity in spaces of weakly differentiable functions. Math. Ann. 313 (1999) 653-710.

[25] M. Kružík and T. Roubíček, On the measures of DiPerna and Majda. Mathematica Bohemica 122 (1997) 383-399.

[26] M. Kružík and T. Roubíček, Optimization problems with concentration and oscillation effects: relaxation theory and numerical approximation. Numer. Funct. Anal. Optim. 20 (1999) 511-530.

[27] C. Licht, G. Michaille and S. Pagano, A model of elastic adhesive bonded joints through oscillation-concentration measures. Prépublication of the Institut de Mathématiques et de Modélisation de Montpellier, UMR-CNRS 5149.

[28] P. Marcellini, Approximation of quasiconvex functions and lower semicontinuity of multiple integrals. Manuscripta Math. 51 (1985) 1-28.

[29] V.G. Mazja, Sobolev Spaces. Springer, Berlin (1985).

[30] N.G. Meyers, Quasi-convexity and lower semicontinuity of multiple integrals of any order. Trans. Am. Math. Soc. 119 (1965) 125-149.

[31] C.B. Morrey, Multiple Integrals in the Calculus of Variations. Springer, Berlin (1966). 
[32] S. Müller, Variational models for microstructure and phase transisions. Lecture Notes in Mathematics 1713 (1999) 85-210.

[33] P. Pedregal, Parametrized Measures and Variational Principles. Birkäuser, Basel (1997).

[34] Yu.G. Reshetnyak, The generalized derivatives and the a.e. differentiability. Mat. Sb. 75 (1968) 323-334 (in Russian).

[35] Yu.G. Reshetnyak, Weak convergence and completely additive vector functions on a set. Sibirsk. Mat. Zh. 9 (1968) 1039-1045.

[36] T. Roubíček, Relaxation in Optimization Theory and Variational Calculus. W. de Gruyter, Berlin (1997).

[37] M.E. Schonbek, Convergence of solutions to nonlinear dispersive equations. Comm. Part. Diff. Equa. 7 (1982) 959-1000.

[38] E.M. Stein, Singular Integrals and Differentiability Properties of Functions. Princeton university Press, Princeton (1970).

[39] L. Tartar, Compensated compactness and applications to partial differential equations, in Nonlinear Analysis and Mechanics, R.J. Knops Ed., Heriott-Watt Symposium IV, Pitman Res. Notes Math. 39, San Francisco (1979).

[40] L. Tartar, Mathematical tools for studying oscillations and concentrations: From Young measures to $H$-measures and their variants, in Multiscale problems in science and technology. Challenges to mathematical analysis and perspectives, N. Antonič et al. Eds., Proceedings of the conference on multiscale problems in science and technology, held in Dubrovnik, Croatia, September 3-9, 2000, Springer, Berlin (2002).

[41] M. Valadier, Young measures, in Methods of Nonconvex Analysis, A. Cellina Ed., Lect. Notes Math. 1446, Springer, Berlin (1990) 152-188.

[42] J. Warga, Optimal Control of Differential and Functional Equations. Academic Press, New York (1972).

[43] L.C. Young, Generalized curves and the existence of an attained absolute minimum in the calculus of variations. Comptes Rendus de la Société des Sciences et des Lettres de Varsovie, Classe III 30 (1937) 212-234. 